\title{
First Measurement of the W Boson Mass WITH CDF IN RUN 2
}

by

\section{Oliver Stelzer-Chilton}

A thesis submitted in conformity with the requirements

for the degree of Doctor of Philosophy

Graduate Department of Physics

University of Toronto

Copyright (C) 2006 by Oliver Stelzer-Chilton 


\author{
Abstract \\ First Measurement of the W Boson Mass \\ with CDF in Run 2 \\ Oliver Stelzer-Chilton \\ Doctor of Philosophy \\ Graduate Department of Physics \\ University of Toronto \\ 2006
}

This thesis describes a first measurement of the $W$ Boson mass through the decay into a muon and a neutrino in Run 2 of the Tevatron. The $W$ Bosons are produced in protonantiproton collisions at a center of mass energy of $1.96 \mathrm{TeV}$. The data sample used for this analysis corresponds to $200 \mathrm{pb}^{-1}$ recorded by the upgraded Collider Detector at Fermilab. The most important quantity in this measurement is the momentum of the muon measured in a magnetic spectrometer which is calibrated using the two quarkonium resonances $\mathrm{J} / \Psi$ and $\Upsilon(1 S)$. Systematic uncertainties arise from the modeling of the recoil when the $W$ Boson is produced, the momentum calibration, the modeling of $W$ Boson production and decay dynamics and backgrounds.

The result is: $M_{W}=80408 \pm 50$ (stat.) \pm 57 (syst.) $\mathrm{MeV} / c^{2}$. 


\section{Acknowledgements}

I would very much like to thank my supervisor William Trischuk for giving me the opportunity to carry out my Ph.D. at the University of Toronto. I could always count on him for his help, advice and support.

Sincere appreciation to Ashutosh Kotwal from Duke University for the countless hours he taught me and for all I learnt as a result. His work and insight into this analysis have been invaluable.

A special thanks also to the other members of the CDF $W$ mass group who directly contributed to this measurement: Ian Vollrath my colleague at the University of Toronto, Chris Hays from Duke University and Larry Nodulman from Argonne National Lab.

The drawing below depicts a humorous drawing of the CDF $W$ mass group. We have always managed to combine work with some fun, especially during our many workshops over the years.

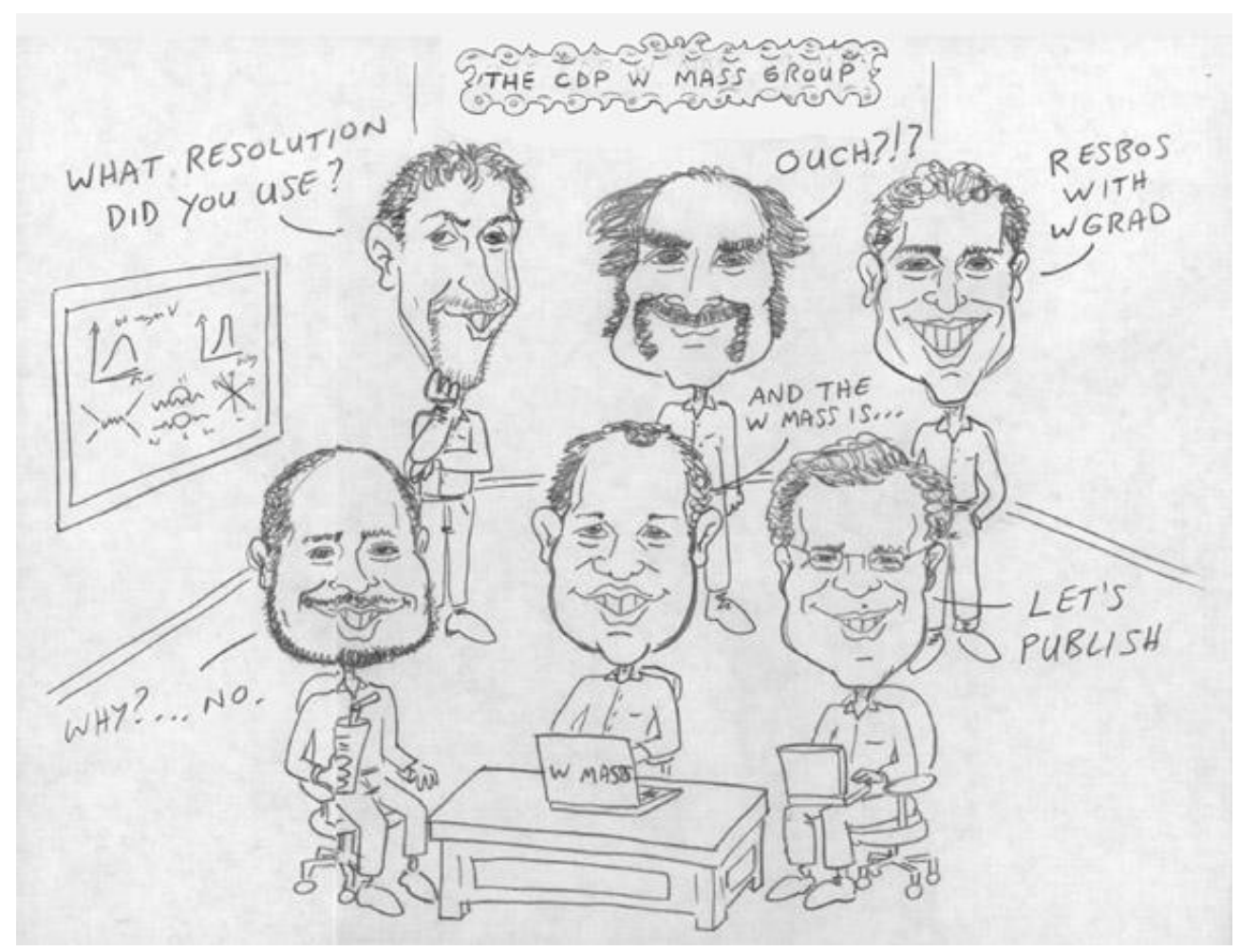

front: Ashutosh, myself and William; back: Chris, Larry and Ian. 
I would like to thank the CDF faculty at the University of Toronto. Pierre Savard, Pekka Sinervo and Bob Orr were always open to my questions.

My fellow post graduates at the University of Toronto were not only fun to hang out with, but also good assistance on several occasions: Stan Lai, Shabnaz Pashapour, JeanFrancois Arguin, Simon Sabik, Reda Tafirout, Rachid Mazini, Dan MacQueen, Kalen Martens, Pierre Beauchemin and of course my brother Bernd Stelzer who has always been a great support.

It is my honour to thank the people of the whole CDF Collaboration, without their combined effort and team work, no analysis would be possible.

My gratitude also to my Ph.D. committee members Peter Krieger and Bob Holdom. Thanks to my wife Ashleigh for her interest in my thesis and her understanding for the sometimes long hours that came with it and to my large family both here and abroad.

I gratefully acknowledge the financial support of the Natural Science and Engineering Research Council of Canada (NSERC), who directly supported me through a graduate student scholarship.

Finally, I would like to thank Canada, now my second home, which I feel always welcomed me with open arms. 


\section{Contents}

1 Introduction $\quad 1$

2 The Standard Model and $W$ Boson Mass Predictions 4

2.1 The Standard Model . . . . . . . . . . . . . . . . . . . . . . . 4

2.2 Predictions for the $W$ Boson Mass . . . . . . . . . . . . . . . . . . . . 7

$2.3 \quad W$ Boson Production . . . . . . . . . . . . . . . . . . . . . . 9

2.4 Measurement Strategy . . . . . . . . . . . . . . . . . . . 15

3 The Accelerator and Detector $\quad 18$

3.1 The Tevatron ........................ 18

3.2 The CDF Detector . . . . . . . . . . . . . . . . . . . . 21

3.2 .1 The Tracking System . . . . . . . . . . . . . . . . . 23

3.2.2 The Calorimeter System . . . . . . . . . . . . 26

3.2 .3 The Muon System . . . . . . . . . . . . . . . . 28

3.2.4 Luminosity Counters . . . . . . . . . . . . . . . . . 31

3.2.5 The Data Acquisition and Trigger System . . . . . . . . . . . . . 32

3.2.6 Detector Operation . . . . . . . . . . . . . . . . 34

3.2.7 Offline Data Reconstruction . . . . . . . . . . . . . . 35

4 Data Sets and Event Selection 36

$4.1 \quad W$ and $Z$ Sample . . . . . . . . . . . . . . . . 37 
4.1 .1 High $p_{T}$ Muon Trigger $\ldots \ldots \ldots \ldots . \ldots . \ldots . \ldots$

4.1 .2 Candidate Selection . . . . . . . . . . . . . . . 38

$4.2 \mathrm{~J} / \Psi$ and $\Upsilon$ Sample . . . . . . . . . . . . . . . . . 41

4.2 .1 Dimuon Trigger . . . . . . . . . . . . . . . . . . . . . . 41

4.2 .2 Candidate Selection . . . . . . . . . . . . . . . . 42

$\begin{array}{lll}5 & \text { Simulation } & 43\end{array}$

5.1 Event Generation . . . . . . . . . . . . . . . . . . . . . . . 44

$5.1 .1 \quad J / \Psi$ and $\Upsilon$ Generation . . . . . . . . . . . . . 44

$5.1 .2 \quad W$ and $Z$ Generation . . . . . . . . . . . 46

5.2 Fast Detector Simulation . . . . . . . . . . . . . . . . . 52

5.2 .1 Simulation of Passive Material . . . . . . . . . . . . . 52

5.2 .2 Tracker Simulation . . . . . . . . . . . . . . . 53

5.2 .3 Muon Acceptance Modeling . . . . . . . . . . . . . . 54

5.2 .4 Calorimeter Response . . . . . . . . . . . . . . . . . . 55

$5.2 .5 \quad$ Underlying Event . . . . . . . . . . . . . . . . 58

$5.2 .6 \quad$ Efficiency Modeling . . . . . . . . . . . . . . . . . . . 60

6 Momentum Calibration $\quad 62$

6.1 Tracker Alignment . . . . . . . . . . . . . . . . . . . . . . 62

6.2 Momentum and $z$ Scales using $J / \Psi \rightarrow \mu \mu$ Events . . . . . . . . 63

6.3 COT Momentum Scale using $\Upsilon \rightarrow \mu \mu$ Events . . . . . . . . . . 67

6.4 Momentum Scale Cross-Check and Momentum Resolution using $Z \rightarrow \mu \mu$ Events ............................ 71

$\begin{array}{lll}7 & \text { Backgrounds } & 73\end{array}$

7.1 Cosmic Rays . . . . . . . . . . . . . . . . . . . . . . . . . 73

7.2 Kaon Decays in Flight _ . . . . . . . . . . . . . . . 76

7.3 QCD Background . . . . . . . . . . . . . . . 77 


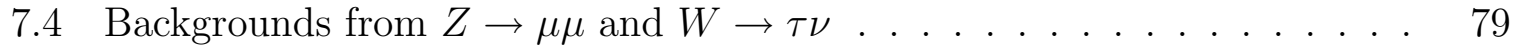

8 Hadronic Recoil Measurement $\quad 84$

8.1 Recoil Response . . . . . . . . . . . . . . . 85

8.2 Recoil Resolution . . . . . . . . . . . . . . . . . . . 86

$8.3 W$ Recoil . . . . . . . . . . . . . . . . . . . . 87

$\begin{array}{llr}9 & \text { Result } & 89\end{array}$

9.1 Fit Results . . . . . . . . . . . . . . . . . . . . . 90

9.2 Summary of Systematic Uncertainties . . . . . . . . . . . . . . 91

9.3 Cross-Checks . . . . . . . . . . . . . . . . . . . . . . . 94

9.4 Comparison to Previous Results . . . . . . . . . . . . . . . . . 95

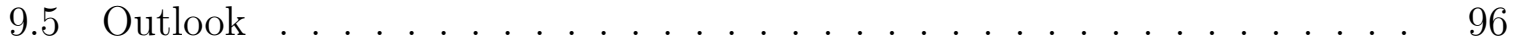

$\begin{array}{ll}\text { Bibliography } & 99\end{array}$ 


\section{List of Tables}

2.1 Fundamental Bosons of the Standard Model. . . . . . . . . . . . . . . . 5

2.2 Fundamental Fermions (quarks and leptons) of the Standard Model. . . . 5

3.1 Accelerator parameters of the Tevatron. . . . . . . . . . . . . . . . 20

4.1 Muon identification requirements. . . . . . . . . . . . . . 39

$4.2 W$ Boson candidate event requirements. . . . . . . . . . . . . . . . . 40

$4.3 Z$ Boson candidate event requirements. . . . . . . . . . . . . . . 40

4.4 Dimuon candidate requirements for $\mathrm{J} / \Psi$ and $\Upsilon$ samples. . . . . . . . . . 42

5.1 Summary of QED systematics on the $W$ Boson mass measurement. . . . 50

6.1 The parameters of the coefficients to the curvature correction function. . 63

6.2 Systematic uncertainties in parts per thousand (ppt) for $\Upsilon(1 S)$ fit . . . . 70

6.3 Measured momentum scales . . . . . . . . . . . . . . . . . 71

7.1 Cosmic background estimates for different normalization regions. . . . . . 75

7.2 Relative acceptances for $W \rightarrow \mu \nu, Z \rightarrow \mu \mu$ and $W \rightarrow \tau \nu \ldots . . . . \quad 81$

7.3 Systematic uncertainty on $W$ mass due to background uncertainty. . . . 83

9.1 Table of systematic uncertainties for the transverse mass fit. When comparing to the Run $1 b$ result, some uncertainties were combined in the Run 1b measurement. . . . . . . . . . . . . . . . . . . 92

9.2 Fitted $W$ Boson mass shifts (in $\mathrm{MeV} / \mathrm{c}^{2}$ ) for sub-divisions of the data. . . 94 
9.3 Current world's best $W$ Boson mass measurements. Note that the LEP experiment results include about $30 \mathrm{MeV} / \mathrm{c}^{2}$ of correlated systematic uncertainty from the LEP beam energy measurement and color recombination

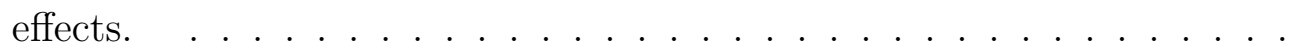




\section{List of Figures}

2.1 Lowest-order radiative corrections to the $W$ Boson mass. . . . . . . . . . 8

2.2 Constraints on the Higgs Boson mass from direct measurements and indirect electroweak predictions for $W$ Boson and top quark mass [19]. . . . . $\quad 9$

2.3 Inferred Higgs Boson mass from all electroweak precision measurements [19]. 10

2.4 a) Feynman diagrams for lowest order s-channel and b) one possible tchannel $W$ Boson production mechanism. . . . . . . . . . . . . . . . 11

$2.5 W$ Boson decay in the center of mass system. . . . . . . . . . . . . . 12

2.6 The $m_{T}$ (left) and $p_{T}$ (right) spectrum for $W$ Bosons with $p_{T}^{W}=0$ (solid line), with the correct $p_{T}^{W} \neq 0$ distribution (points), and with detector resolution (shaded) [23]. . . . . . . . . . . . . . . . . . 14

2.7 Transverse mass template for $80 \mathrm{GeV} / \mathrm{c}^{2}$ (blue histogram) and $81 \mathrm{GeV} / \mathrm{c}^{2}$ (red histogram). . . . . . . . . . . . . . . . 16

3.1 Schematic of the Tevatron accelerator chain. . . . . . . . . . . . . . . 19

3.2 Schematic of the CDF detector. . . . . . . . . . . . . . . . 22

3.3 The CDF coordinate system. . . . . . . . . . . . . . . . 23

3.4 Schematic of the CDF tracking volume. . . . . . . . . . . . . . 23

3.5 Cell configuration for superlayer 2 of the COT. . . . . . . . . . . . 25

$3.61 / 6$ th view of the COT, including the radii $(\mathrm{cm})$ of each superlayer. . . . 25

3.7 Schematic of a wedge in the central calorimeter. . . . . . . . . . . . . 27

3.8 Coverage $(\eta, \phi)$ of the CDF central muon system. . . . . . . . . 28 
3.9 Schematic of one wedge of the CMU chambers. . . . . . . . . . . . . 29

3.10 Transverse view of a CMU module. . . . . . . . . . . . . . . . . 30

3.11 Transverse view of a CMP stack. . . . . . . . . . . . . . . . 30

3.12 Drift chamber layout in a CMX module. The eight layers of chambers are grouped in pairs to form four continuous layers. . . . . . . . . . . . . 31

3.13 Diagram of the CDF dataflow (Rates quoted are design parameters). . . 33

5.1 The $p_{T}^{J / \Psi}$ distribution straight out of PYTHIA. . . . . . . . . . . . . 45

5.2 The $p_{T}^{J / \Psi}$ distribution after tuning. . . . . . . . . . . . . . 45

5.3 Kinematics in the quarkonium rest frame. . . . . . . . . . . . . . . 46

5.4 The sum of the curvature distribution straight out of PYTHIA. . . . . . 46

5.5 The sum of the curvature distribution after $\theta^{*}$ tuning is applied. . . . . . 46

$5.6 p_{T}^{W}$ spectrum generated by RESBOS for various $g_{2}$ values. The mean $W p_{T}$ increases as $g_{2}$ increases. . . . . . . . . . . . . . . . . 47

5.7 Projection of $\sqrt[3]{k}$. Events with no photons are in the zero bin. . . . . . . 49

5.8 Projection of $\sqrt{\Delta R(\mu, \gamma)}$. Events with $\Delta R>1$ are in the last bin. . . . . 49

5.9 Variation of the twenty CTEQ6 pdf error pairs. . . . . . . . . . . 51

5.10 a) Probability of finding a CMUP muon as a function of $z_{0}$ and $\cot \theta$ and b) probability of finding a CMX muon as a function of $\phi_{0}$ and $\cot \theta$. . . 54

5.11 a) The comparison of data and simulation $\cot \theta$ and b) $\phi_{0}$ distributions. . 55

5.12 Instantaneous luminosity for minimum bias, $W$ and $Z$ events. . . . . . . 57

5.13 a) The mean $E_{T}$ in the electromagnetic calorimeter towers and b) hadronic calorimeter towers in the vicinity of the muon track. . . . . . . . 58

5.14 Illustration of momentum vectors in the transverse plane and components of the $u$ vector parallel and perpendicular to the muon. . . . . . . . . . . 59

5.15 Variation of $E_{T}^{u e}$ in the removed muon towers with $\eta$. . . . . . . . . . 60

5.16 Variation of $E_{T}^{u e}$ in the removed muon towers with $u_{\|} \ldots \ldots$. . . . 60

5.17 Variation of muon identification efficiency with $u_{\|} \ldots \ldots$. . . . . . . . 61 
6.1 Template for $J / \Psi$ momentum scale fit. . . . . . . . . . . . . . . . 64

6.2 Fitted $\Delta \mathrm{p}$ as a function of $\Delta \cot \theta$, before corrections, fitted to a parabola. 65

6.3 Fitted $\Delta \mathrm{p}$ as a function of $\Delta \cot \theta$, after corrections, fitted to a parabola. $\quad 65$

6.4 Fitted $\Delta \mathrm{p}$ as a function of $\left\langle 1 / p_{T}^{\mu}\right\rangle$, fitted to a straight line. . . . . . 66

6.5 Dimuon mass spectrum with a fit of three Gaussian distributions and a linear background . . . . . . . . . . . . . . . . . 67

6.6 Comparison of data with Monte Carlo of the $\Upsilon(1 \mathrm{~S}) p_{T}$ distribution. . . . 68

6.7 Comparison of data with Monte Carlo of the $\Upsilon(1 \mathrm{~S}) p_{z}$ distribution. . . . 68

6.8 Comparison of the muon candidate $p_{T}$ distribution. . . . . . . . 68

6.9 Comparison of the muon candidate $p_{z}$ distribution. $\ldots \ldots \ldots 6$

6.10 The invariant mass distribution with the best fit simulated template and the extracted momentum scale using non beam constrained quantities. . 69

6.11 The invariant mass distribution with the best fit simulated template and the extracted momentum scale using beam constrained quantities. . . . . 69

6.12 The $Z \rightarrow \mu \mu$ invariant mass fit. . . . . . . . . . . . . 72

$7.1 t_{0}$ distribution for the outgoing track for all signal events. . . . . . . 74

$7.2 t_{0}$ distribution for the outgoing track for $d_{0}$ sideband events. . . . . . . 74

7.3 $M_{T}$ distribution for cosmic background. The best fit to the data and the $3 \sigma$ fitted shape uncertainty are overlaid. . . . . . . . . . 75

7.4 $M_{T}$ distribution for kaon background. The best fit to the data and the $3 \sigma$ fitted shape uncertainty are overlaid. . . . . . . . . . . . 76

$7.5 \chi^{2}$ fit for $1.7 \%$ QCD shape added in the $\not_{T}$ distribution. $\ldots \ldots \ldots 78$

$7.6 \chi^{2}$ fit for $0.9 \%$ QCD shape added to calorimeter isolation ratio distribution. 78

7.7 $M_{T}$ distribution for QCD background. The best fit to the data and the $3 \sigma$ fitted shape uncertainty are overlaid. . . . . . . . . . . . 79

7.8 Events rejected by $Z$ veto with $Z \rightarrow \mu \mu$ Monte Carlo overlaid. . . . . . . 80 
7.9 $M_{T}$ distribution for $Z \rightarrow \mu \mu$ background. The best fit to the data and the $3 \sigma$ fitted shape uncertainty are overlaid. . . . . . . . . . . . 82

7.10 $M_{T}$ distribution for $W \rightarrow \tau \nu$ background. The best fit to the data and the $3 \sigma$ fitted shape uncertainty are overlaid. . . . . . . . . . . . . 82

$7.11 M_{T}$ distributions for all backgrounds and total background overlaid. . . . 83

8.1 Distribution of $p_{T}(\mu \mu)$ in $Z \rightarrow \mu \mu$ events. . . . . . . . . . . 84

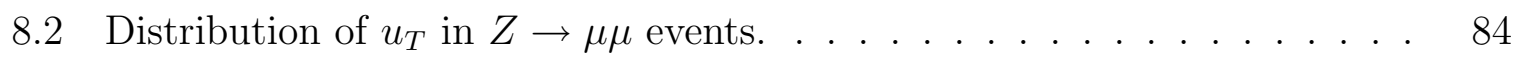

8.3 Illustration of momentum vectors in the transverse plane for $Z \rightarrow \mu \mu$ events. 85

8.4 The mean value of the $p_{T}$-balance in the $\eta$ direction in $Z \rightarrow \mu \mu$ events. The dots represent the data, the histogram the simulation. . . . . . . 86

8.5 The rms value of the $p_{T}$-balance in the $\eta$ direction in $Z \rightarrow \mu \mu$ events. . . 87

8.6 Distribution of $u_{T}$ in $W \rightarrow \mu \nu$ events. Background contributions are included in the simulated distribution. . . . . . . . . 87

8.7 Distribution of $\Delta \phi(\mu, u)$ in $W \rightarrow \mu \nu$ events. Background contributions are included in the simulation. . . . . . . . . . . . 87

8.8 Distribution of $u_{\|}$in $W \rightarrow \mu \nu$ events. Background contributions are included in the simulated distribution. . . . . . . . . . 88

8.9 Distribution of $u_{\perp}$ in $W \rightarrow \mu \nu$ events. Background contributions are included in the simulated distribution. . . . . . . . . 88

9.1 Transverse mass template used to extract the $W$ Boson mass. . . . . . . 89

9.2 The $W \rightarrow \mu \nu$ transverse mass fit. . . . . . . . . . . . . . . . . 91

9.3 Normalized $\chi$ distribution for the $W$ mass fit. . . . . . . . . . . . . 95

9.4 Projected $W$ Boson mass precision with integrated luminosity [55]. The black markers shows previously achieved precisions from CDF and DØ, the blue arrows indicate the anticipated precision from CDF for $200 \mathrm{pb}^{-1}$ and $2 \mathrm{fb}^{-1} \ldots \ldots \ldots \ldots \ldots \ldots \ldots \ldots \ldots \ldots \ldots \ldots \ldots \ldots \ldots \ldots$ 


\section{Chapter 1}

\section{Introduction}

The massive charged $W^{ \pm}$and neutral $Z$ Bosons were proposed in the 1960s as the mediators of the weak force. The large $W$ Boson mass was proposed to explain the observed point-like interaction in $\beta$ decay. Glashow incorporated these particles in a gauge theory unifying the weak and electromagnetic interactions [1]. To be gauge invariant the orig-

inal theory required massless Bosons. Weinberg [2] and Salam [3] showed that massive Bosons can be incorporated into a gauge invariant theory through the Higgs mechanism [4]. This theory developed into what is now known as the Standard Model of particle physics. The phenomenology of charged weak interactions was well established at the time, but the neutral weak current had not yet been observed. The indirect discovery of the $Z$ Boson in the CERN Gargamelle bubble chamber through neutrino scattering [5] was the first success of the Standard Model. The Standard Model made firm predictions for the masses of the proposed Bosons. The charged Boson was predicted to have a mass of $70-80 \mathrm{GeV} / \mathrm{c}^{2}$ and the neutral Boson $80-90 \mathrm{GeV} / \mathrm{c}^{2}$.

On the experimental side, Rubbia proposed to turn the CERN hadron accelerator $S p S$ into a proton-antiproton collider. The $S p \bar{p} S$ proton-antiproton collider was commissioned in late 1981, and in 1983, the UA1 [6] and UA2 [7] experiments reported the observation of the $W$ Bosons. 
A few years later, in 1985, the world's second proton-antiproton collider, the Tevatron at Fermilab, was commissioned, operating at approximately three times the $S p \bar{p} S$ center of mass energy. In 1987 the first $W$ candidates were observed at Fermilab [8]. Until 1995, the production and study of the $W$ Boson was the exclusive domain of the $p \bar{p}$ colliders at CERN and Fermilab. Beginning in 1996, CERN's electron-positron collider, LEP ${ }^{1}$, increased its center of mass energy above $161 \mathrm{GeV}$ which is the threshold for $W$ pair production. Since then, the $W$ Boson mass has been measured with increasing precision by the two Tevatron experiments, CDF and DØ [9], and the four LEP experiments, ALEPH, DELPHI, L3 and OPAL which ceased data-taking in 2000 [10].

A precision measurement of the $W$ Boson mass is important since it is a fundamental parameter of the Standard Model. Higher-order radiative corrections from loop diagrams involving other particles contribute to the observed $W$ Boson mass. The most important corrections come from the top quark and the Higgs Boson. Since the Higgs Boson has not yet been observed, precise measurements of the $W$ Boson mass and the top quark mass constrain the mass of the Higgs Boson. During the Tevatron's previous collider runs, the $W$ Boson mass was measured to be $80.454 \pm 0.059 \mathrm{GeV} / \mathrm{c}^{2}[9]$ and the top quark mass was measured to be $178.0 \pm 4.3 \mathrm{GeV} / \mathrm{c}^{2}[11]$. With the Tevatron Run 2 dataset it will be possible to measure the $W$ Boson mass with a precision of $\sim 30 \mathrm{MeV} / \mathrm{c}^{2}$ and the top quark mass to within $\sim 2 \mathrm{GeV} / \mathrm{c}^{2}$, which will constrain the Higgs Boson mass to a relative uncertainty of $\sim 30 \%$. This will substantially narrow the window for a direct search of the Higgs Boson.

This thesis describes the analysis of the first $200 \mathrm{pb}^{-1}$ of CDF Run 2 data and is organized as follows. Chapter 2 describes the Standard Model of particle physics and the theoretical predictions for the $W$ Boson mass. It also discusses the $W$ Boson production mechanism at hadron colliders and outlines the measurement strategy. In chapter 3 the accelerator and detector apparatus is described, with an emphasis on aspects important

\footnotetext{
${ }^{1}$ Large Electron-Positron collider
} 
for this analysis. Chapter 4 outlines the data sets and explains the event selection. Simulation of $W$ production, which includes event generation, and detector simulation are described in chapter 5. The calibration of the CDF momentum scale is explained in chapter 6 . This is followed by a description of the background calculation for the $W$ Boson samples in chapter 7 and a description of the $W$ Boson recoil model in chapter 8 . Chapter 9 presents the final result and a summary. 


\section{Chapter 2}

\section{The Standard Model and $W$ Boson Mass Predictions}

\subsection{The Standard Model}

Experimental discoveries and theoretical developments over the last 50 years have led to the development of the Standard Model of particle physics. Since it is the subject of many articles and books [12][13], this chapter provides only a brief summary. The Standard Model is a field theory with local gauge invariance and describes all of the observed fundamental particles and their interactions. These include the electromagnetic interaction, the weak interaction and the strong interaction. The gravitational interaction, which is by far the weakest of the four fundamental interactions, is not described by the Standard Model. Table 2.1 gives an overview of the four fundamental interactions.

The particles can be grouped according to their spin. Half-spin particles are called Fermions, gauge Bosons carry spin 1 and the Higgs Boson 0. Matter is made out of Fermions. The objects we come across in our everyday lives basically consist of three particles, the up and down quarks and the electron. For unknown reasons, this pattern of basic building blocks is repeated three times, making three generations of fundamental 


\begin{tabular}{|c|c|c|c|}
\hline \multicolumn{4}{|c|}{ Fundamental interactions and gauge Bosons } \\
\hline Interaction & Gauge Bosons & Gauge Group & Acts on \\
\hline Electromagnetic & Photon $(\gamma)$ & $\mathrm{U}(1)$ & electric charge \\
Weak & $W^{ \pm}, Z^{0}(W)(Z)$ & $\mathrm{SU}(2)$ & flavor \\
Strong & gluons $(\mathrm{g})$ & $\mathrm{SU}(3)$ & color charge \\
Gravity & graviton $(\mathrm{G})$ & & mass \\
\hline
\end{tabular}

Table 2.1: Fundamental Bosons of the Standard Model.

Fermions. Table 2.2 gives a summary of all Fermions, which are subdivided into quarks and leptons. The down-type quarks $(d, s, b)$ carry electric charge $-\frac{1}{3} e$ and the up-type quarks $(u, c, t)$ carry electric charge $+\frac{2}{3} e$, where $e$ is the absolute charge of the electron. Each lepton generation consists of a charged particle (electron, muon or tau) and an associated neutrino. The charged leptons carry electric charge $-1 e$ while the neutrinos have no electric charge. Recent results provide evidence that neutrinos have masses that are small but finite [14].

\begin{tabular}{|c|c|c|c|c|}
\cline { 2 - 5 } \multicolumn{1}{c|}{} & \multicolumn{4}{|c|}{ Fundamental Fermions (Spin-1/2) } \\
\cline { 2 - 5 } \multicolumn{1}{c|}{ Quarks } & \multicolumn{2}{c|}{ Leptons } \\
\hline Gen. & Flavor & Charge & Flavor & Charge \\
\hline I & Up $(u)$ & $+2 / 3$ & Electron neutrino $\left(\nu_{e}\right)$ & 0 \\
& Down $(d)$ & $-1 / 3$ & Electron $\left(e^{-}\right)$ & -1 \\
\hline II & Charm $(c)$ & $+2 / 3$ & Muon neutrino $\left(\nu_{\mu}\right)$ & 0 \\
& Strange $(s)$ & $-1 / 3$ & Muon $\left(\mu^{-}\right)$ & -1 \\
\hline III & Top $(t)$ & $+2 / 3$ & Tau neutrino $\left(\nu_{\tau}\right)$ & 0 \\
& Bottom $(b)$ & $-1 / 3$ & Tau $\left(\tau^{-}\right)$ & -1 \\
\hline
\end{tabular}

Table 2.2: Fundamental Fermions (quarks and leptons) of the Standard Model. 
Neutrinos interact only through the weak force while the charged leptons can also interact through the electromagnetic force. The strong, weak and electromagnetic interactions are very similar in their structure. This is shown in the most basic interaction between two particles, which can be represented as a process in which two particles exchange a vector Boson. In mathematical terms, the Lagrangian of each interaction obeys gauge invariance under a transformation that corresponds to a symmetry group. The Standard Model is based on the symmetry group $S U(3) \times S U(2) \times U(1)$ [16]. Quantum ElectroDynamics (QED) is based on the $U(1)$ symmetry group and describes electromagnetic interactions among quarks and leptons through the exchange of photons [17]. The strong interaction is described by a gauge field theory called Quantum ChromoDynamics (QCD) [18], which has been developed by analogy to QED. Eight gauge Bosons, called gluons, mediate the interaction of particles carrying color charge. A quark can occur in three different colors and a gluon always carries one color and an anti-color. The fact that the gauge Bosons themselves are colored objects distinguishes them from QED where the photons do not carry charge and makes it possible for the gluons to interact with each other.

The weak interaction is mediated by the $W^{ \pm}$and $Z$ Bosons. Since these particles are very massive, they can only interact over small distances. One of the greatest achievements in the development of the Standard Model was the unification of the electromagnetic and weak interactions to form the electroweak theory [1]-[3]. This unification required the introduction of a complex scalar field, the presence of which breaks the symmetry of the $\mathrm{SU}(2) \times \mathrm{U}(1)$ gauge group. The process of symmetry breaking allows the $W^{ \pm}$and $Z$ Bosons to acquire mass in a gauge invariant way [4].

The Higgs field is a complex doublet with four real components. All particles interact with this field and therefore behave as though having an effective mass, the value of which depends on the strength of the coupling to the Higgs field. Three degrees of freedom of the postulated Higgs field are used to describe the three longitudinal degrees of freedom 
for the spins of the three massive gauge Bosons $W^{ \pm}$and $Z$. The fourth degree of freedom corresponds to an excited state of the Higgs field which should, if this field exists, be observable as a massive scalar particle, known as the Higgs Boson. The Higgs Boson is the last missing particle of the Standard Model, which has not yet been discovered.

\subsection{Predictions for the $W$ Boson Mass}

The mass of the $W$ Boson is sensitive to the mass of the Higgs Boson through radiative corrections. These are quantum corrections where the $W$ Boson can disintegrate into other particles, including the Higgs Boson. Although this may be forbidden kinematically, energy conservation can be violated for a short amount of time according to the Heisenberg uncertainty principle.

At lowest order, the $W$ Boson mass can be predicted from three precisely measured Standard Model quantities: the mass of the $Z$ Boson; the Fermi constant $G_{F}$; and the electromagnetic coupling constant $\alpha$. The Fermi constant, $G_{F}$, is related to the weak coupling constant, $g$, by:

$$
g^{2}=(8 / \sqrt{2}) G_{F} M_{W}^{2}
$$

The weak coupling constant, $g$, is related to the electromagnetic coupling, $e$, through the weak mixing angle $\theta_{W}$ :

$$
g \cdot \sin \left(\theta_{W}\right)=e
$$

By inserting (1) into (2), one obtains the Standard Model prediction for the $W$ Boson mass in terms of the three parameters.

$$
M_{W}=\left(\frac{\pi \alpha}{\sqrt{2} G_{F}}\right)^{1 / 2} \frac{1}{\sin \left(\theta_{W}\right) \sqrt{1-\Delta r}}
$$

where $\Delta r[15]$ parametrizes the effect of the radiative corrections (not included in (1) and (2)). From the measured $W$ Boson mass, one can measure $\Delta r$. Within the Standard Model one can predict the contribution from Fermion loops and the Higgs Boson mass. 
Since the correction from the Fermion loops depends on the squared quark mass differences, the correction from the $t \bar{b}$ quark loop dominates. Figure 2.1 shows the lowest-order loop corrections for a Higgs Boson and a $t \bar{b}$ quark loop. Since the top quark mass has

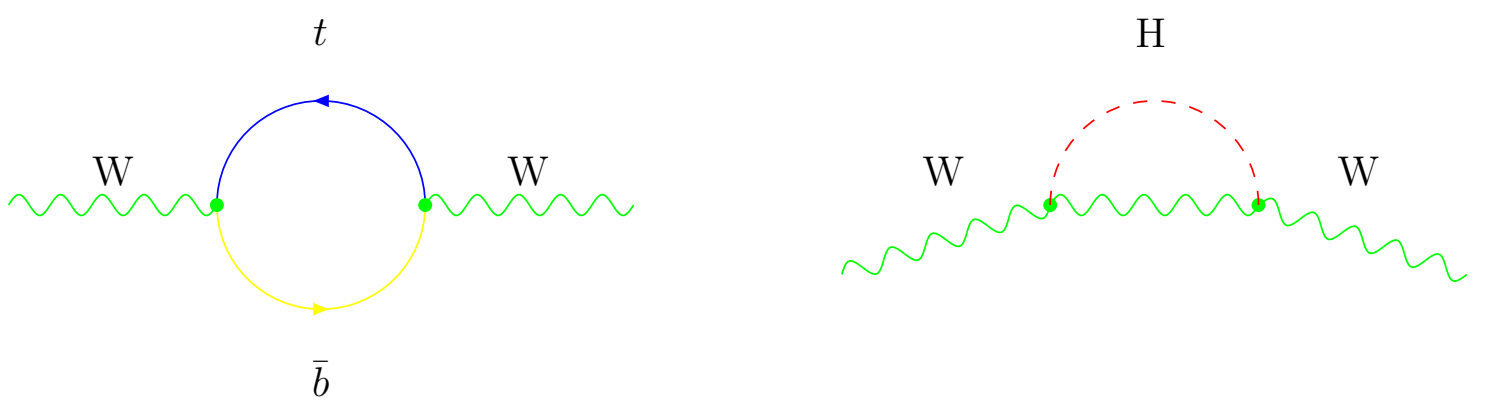

Figure 2.1: Lowest-order radiative corrections to the $W$ Boson mass.

been measured, its contribution can be calculated. Thus a measurement of the $W$ Boson mass constrains the Higgs Boson mass. Figure 2.2 shows the constraint on the Higgs Boson mass from the current world averages of the measured $W$ Boson mass and top quark mass. Higgs Boson masses below $114 \mathrm{GeV} / \mathrm{c}^{2}$ are excluded from direct searches at LEP. The indirect predictions from electroweak measurements and the Standard Model are also shown. There is a reasonable overlap of the two contours. Direct measurements of $W$ Boson and top quark mass favor a relatively low Higgs Boson mass as does the region of overlap between the direct and indirect measurements. Figure 2.3 shows the $\Delta \chi^{2}$ curve derived from electroweak measurements as a function of the Higgs Boson mass, assuming the Standard Model to be the correct theory of nature. The world averages of the $W$ Boson mass and the top quark mass are included together with constraints from other electroweak measurements of $Z$ Boson properties at LEP and SLC ${ }^{1}$, The most probable value for the Higgs Boson obtained is: $M_{\text {Higgs }}=129_{-49}^{+74} \mathrm{GeV} / \mathrm{c}^{2}[19]$.

\footnotetext{
${ }^{1}$ Stanford Linear Collider
} 


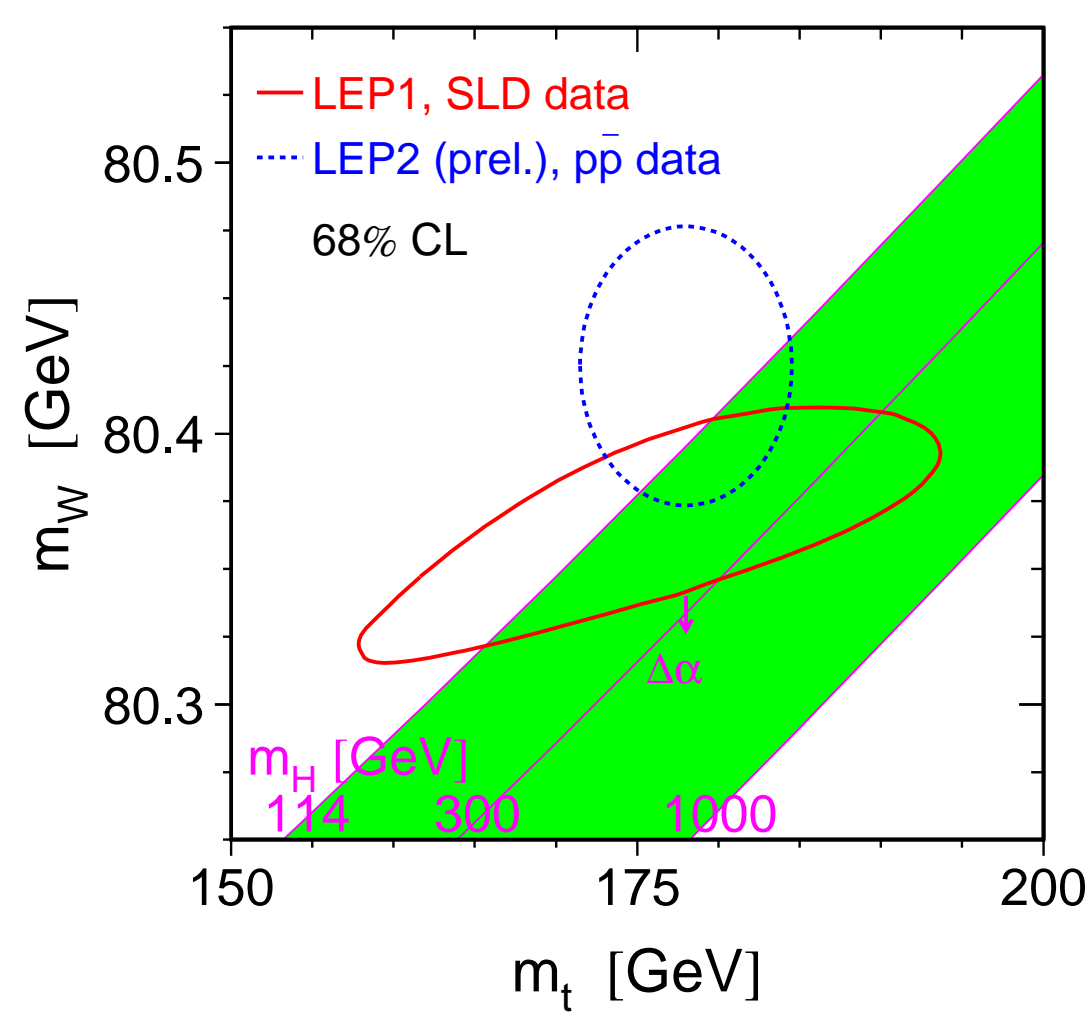

Figure 2.2: Constraints on the Higgs Boson mass from direct measurements and indirect electroweak predictions for $W$ Boson and top quark mass [19].

\section{3 $W$ Boson Production}

In proton-antiproton $(p \bar{p})$ collisions, the four-momentum of the $p$ and $\bar{p}$ is shared among the quarks and gluons inside the proton, collectively known as partons. In inelastic collisions with high momentum transfer, individual partons inside the proton/antiproton undergo an interaction which breaks up the initial proton and antiproton. The energy available in the collision, which produces new particles like the $W$ Boson, is only a fraction of the total center-of-mass energy of the $p \bar{p}$ system. If $P_{1}$ is the four-momentum of the proton and $P_{2}$ is the four-momentum of the antiproton and $x_{1}$ and $x_{2}$ are the fractions of momentum carried by the partons which undergo the interaction, then the square of 


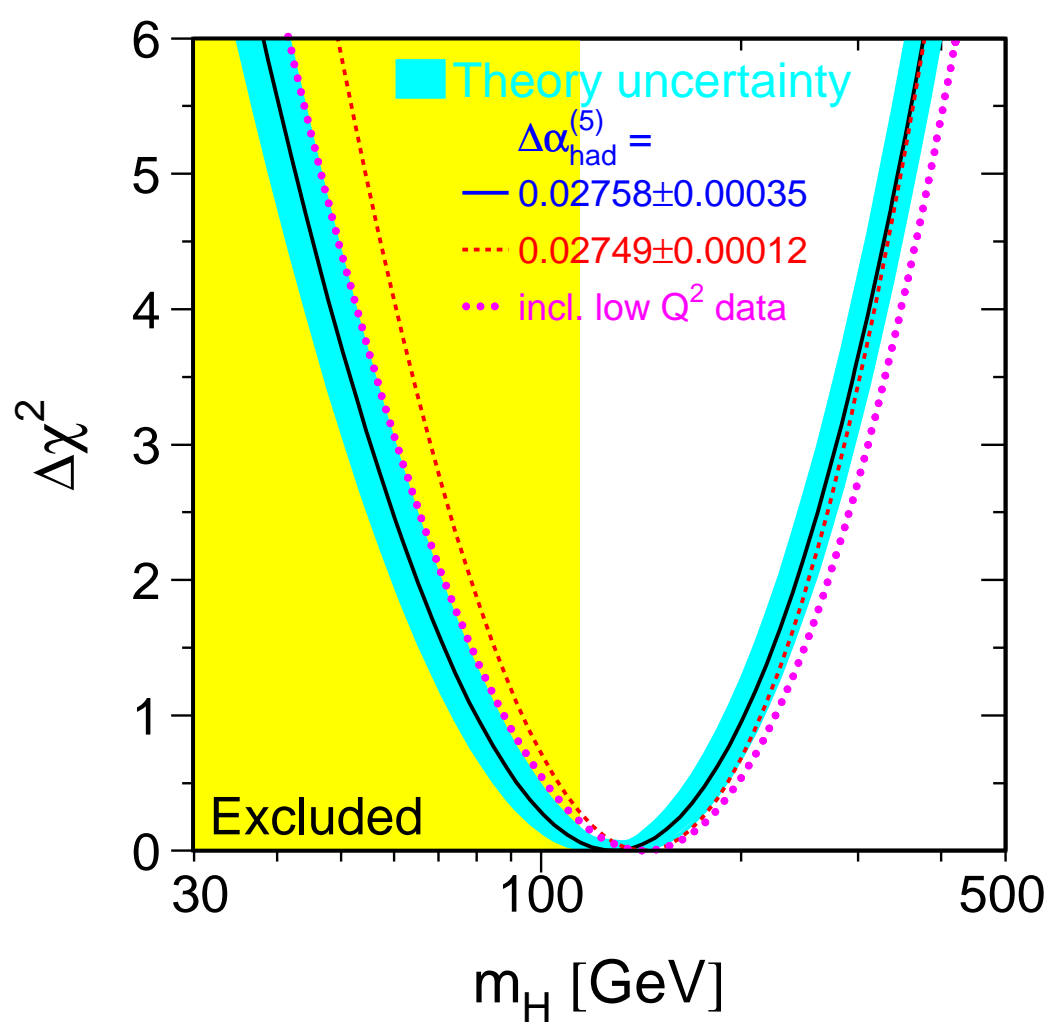

Figure 2.3: Inferred Higgs Boson mass from all electroweak precision measurements [19].

the invariant mass of the parton system, $\hat{s}$, can be expressed as:

$$
\hat{s}=\left(x_{1} P_{1}+x_{2} P_{2}\right)^{2} \simeq 2 x_{1} x_{2} P_{1} P_{2} \simeq x_{1} x_{2}\left(P_{1}+P_{2}\right)^{2}=x_{1} x_{2} s,
$$

where $s$ is the square of the invariant mass of the proton-antiproton system. The masses of the proton and antiproton are assumed to be negligible in this calculation.

The fraction of momentum $(x)$ carried by the individual partons inside the proton is characterized by the parton distribution functions (PDFs). The probability of finding a certain parton with momentum fraction between $x$ and $x+d x$ is $f(x) d x$, where $f(x)$ represents the PDF. The PDFs have been measured in deep inelastic electron-proton scattering mainly by the $\mathrm{H} 1$ and ZEUS Collaborations at DESY ${ }^{2}$. Two sets of PDFs,

\footnotetext{
${ }^{2}$ Deutsches Elektronen-Synchrotron
} 
prepared by the CTEQ [20] and MRS [21] analysis groups are currently available. At the Tevatron, $W$ and $Z$ Bosons are mainly produced through quark anti-quark annihilation, as the quarks carry the largest momentum fraction, $x$, of the proton. The momentum fraction carried by the annihilating partons is inevitably unequal, giving the $W$ Boson a net longitudinal momentum: $p_{L}=\left(x_{1}-x_{2}\right) \sqrt{s}$. This can result in a large longitudinal momentum and due to the finite acceptance of the detector is unmeasured. The event kinematics in $W$ Boson candidate events are therefore usually studied in the plane perpendicular to the beam where all momenta are well measured. Figure 2.4 shows two lowest order $W$ Boson production diagrams. Owing to its clean experimental signature, we study the leptonic $W$ Boson decay into a muon and a neutrino. The neutrino from the $W$ Boson decay interacts only through the weak interaction and therefore is invisible to the detector. There is practically no motion in the transverse direction with respect to

a)

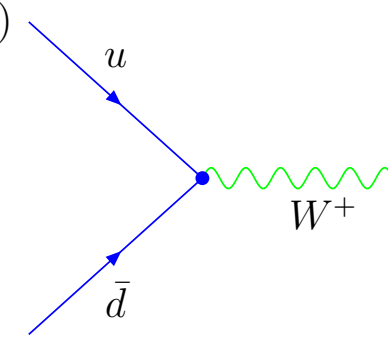

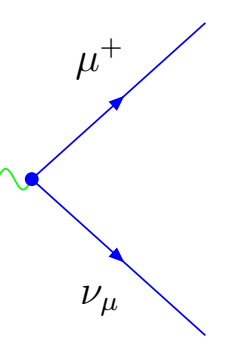

b)

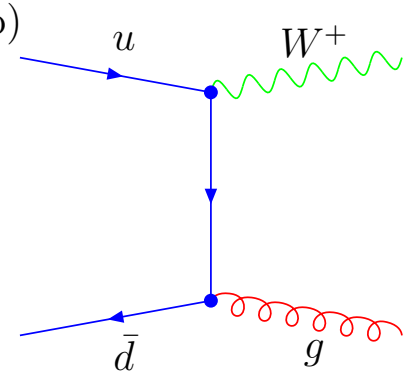

Figure 2.4: a) Feynman diagrams for lowest order s-channel and b) one possible t-channel $W$ Boson production mechanism.

the proton beam before the collision (apart from a small "Fermi motion" of the quarks inside the proton) so that after the collision, there should be no net transverse momentum. This allows one to compute the transverse momentum of the neutrino by momentum conservation. In the simplest case where the $W$ Boson has no transverse momentum, the neutrino and the muon have equal transverse momentum. However the $W$ Boson itself is produced with transverse momentum originating from QCD processes like the one shown on the right side of Figure 2.4. The transverse momentum of the $W$ Boson is balanced by the transverse momentum of hadrons produced with the original collision. The summed 
transverse momentum of these hadrons are usually referred to as the "recoil" momentum. By measuring the total detected transverse momentum (muon+recoil) and requiring its conservation, one can infer the neutrino transverse momentum.

At lowest order, the angular distribution of the muon from $W$ Boson decay is described by the V-A nature of the weak interaction:

$$
\frac{d \sigma}{d \cos \theta} \propto(1-\lambda \cos \theta)^{2},
$$

where $\lambda$ is the helicity of the $W$ Boson with respect to the proton direction and $\theta$ is the angle between the charged muon and the beam direction in the $W$ Boson rest frame. Figure 2.5 shows the production of a $W$ Boson and its decay at lowest order. The $W$

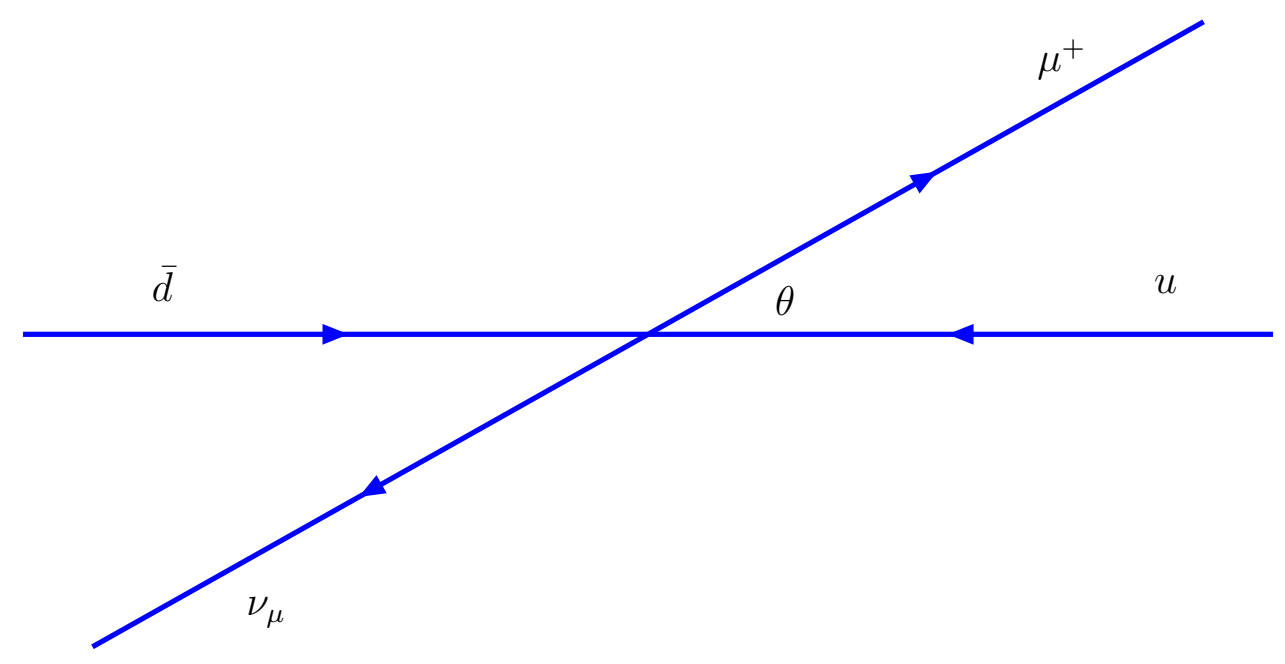

Figure 2.5: $W$ Boson decay in the center of mass system.

Boson spin is aligned with the direction of the incoming valence antiquark. QCD quark and gluon radiation off the initial parton has two effects on the $W$ decay. Firstly, the $W$ spin axis is no longer the beam axis. Secondly, the initial state angular momentum is altered because the radiated partons carry away their spin angular momentum. The $W$ decay angular distribution is modified by these effects in that it depends on the $W$ Boson transverse momentum $p_{T}^{W}$

$$
\frac{d \sigma}{d \cos \theta_{C S}} \propto\left(1-\lambda \alpha_{1}\left(p_{T}^{W}\right) \cos \theta_{C S}+\alpha_{2}\left(p_{T}^{W}\right) \cos ^{2} \theta_{C S}\right),
$$


where the coefficients $\alpha_{i}$ are a function of $p_{T}^{W}$, and where $\theta_{C S}$ is the decay angle in the Collins-Soper frame ${ }^{3}[22]$. If the $W$ Boson decay axis is perpendicular to the beam axis, the muon receives a large transverse momentum due to the $W$ Boson mass. This leaves a clear signature of the $W$ Boson decay in the detector: a muon with large transverse momentum and missing energy resulting from the neutrino.

Since the neutrino momentum component in the direction of the beam is unknown, we cannot reconstruct the invariant mass of the muon-neutrino parent: the $W$ Boson candidate transverse mass, $m_{T}$, is similar to the invariant mass, using only the two dimensions perpendicular to the beam:

$$
m_{T}=\sqrt{2 p_{T}^{\mu} \cdot p_{T}^{\nu}(1-\cos (\Delta \phi))},
$$

where $p_{T}^{\mu}$ represents the transverse momentum of the muon, $p_{T}^{\nu}$ the transverse momentum of the neutrino and $\Delta \phi$ the angle between the two transverse momenta. The transverse mass spectrum is relatively insensitive to the production dynamics of the $W$ Boson. Corrections to the transverse mass due to the motion of the $W$ Boson are only quadratic $\left(p_{T}^{W} / M_{W}\right)^{2}[23]$. However, the transverse mass makes use of the inferred neutrino transverse momentum and is therefore sensitive to the response of the detector to the recoil particles.

An alternative way to measure the $W$ Boson mass is to use the muon transverse momentum spectrum. It is measured with better resolution than the neutrino transverse momentum and is thus insensitive to the recoil response of the detector. It is however more sensitive to the $W$ Boson production dynamics and the corrections due to the motion of the $W$ Boson are linear $\left(p_{T}^{W} / M_{W}\right)$. These effects are illustrated in Figure 2.6, which shows the effect of $p_{T}^{W}$ and the detector resolution on the $m_{T}$ and $p_{T}^{\mu}$ distributions. The solid lines show the shape of the distributions before the detector simulation with $p_{T}^{W}=0$. The points show the distributions when $p_{T}^{W} \neq 0$ and the shaded histogram

\footnotetext{
${ }^{3}$ The Collins-Soper frame is a $W$ rest frame in which the $z$-axis is defined as the bisector of the angle between the proton and antiproton momentum vectors.
} 

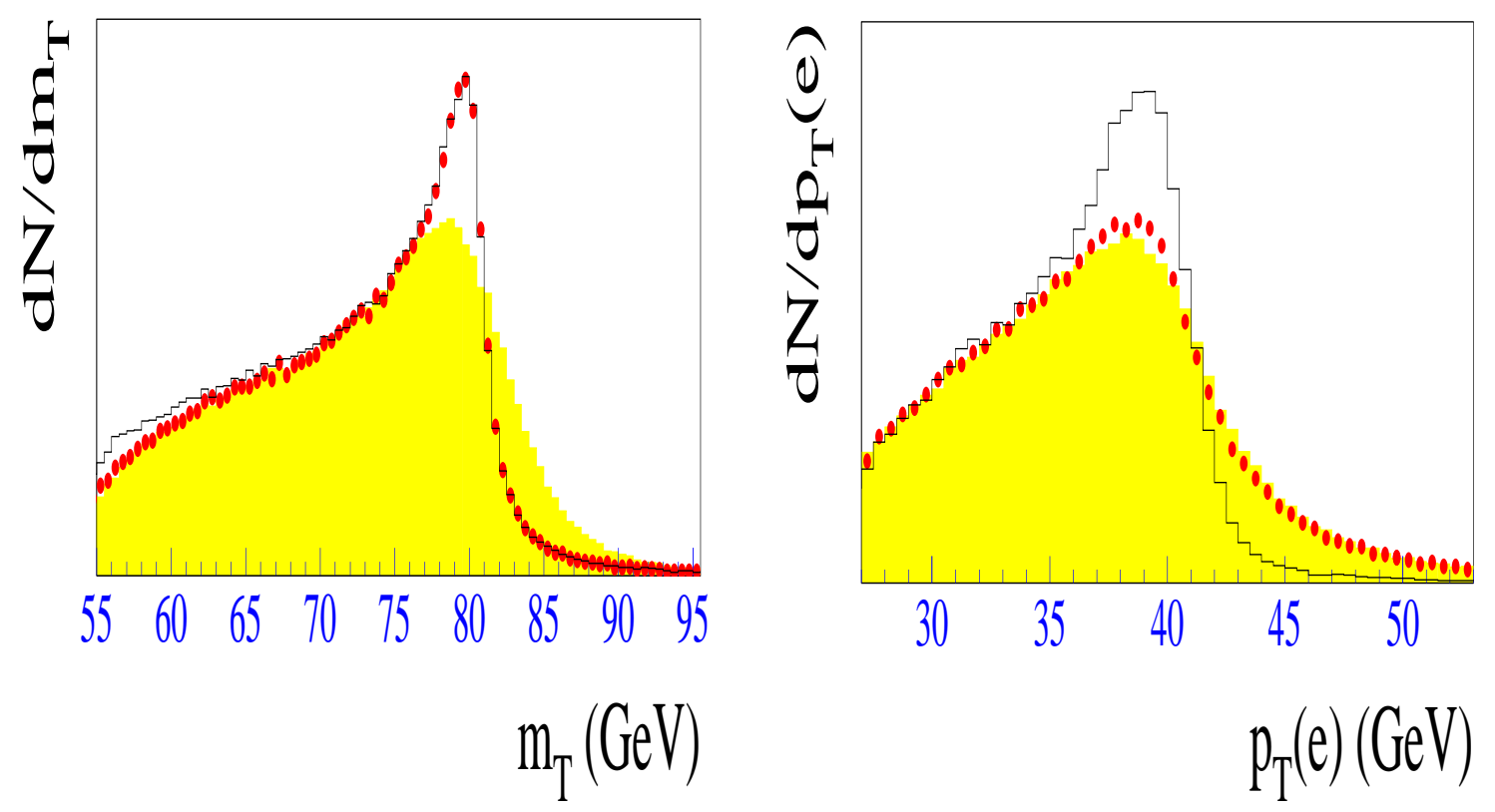

Figure 2.6: The $m_{T}$ (left) and $p_{T}$ (right) spectrum for $W$ Bosons with $p_{T}^{W}=0$ (solid line), with the correct $p_{T}^{W} \neq 0$ distribution (points), and with detector resolution (shaded) [23].

includes a detector simulation. In summary, the $m_{T}$ spectrum is dominated by detector resolution, whereas the $p_{T}^{\mu}$ spectrum is dominated by the motion of the $W$ Boson.

The neutrino transverse momentum spectrum can also be used to extract the $W$ Boson mass. It is sensitive to both the $W$ production dynamics and the recoil measurement.

In principle, the effect of one or more gluon radiations (see Figure 2.4) can be calculated within the context of QCD. However, at low transverse momenta, the gluons radiated are very soft and QCD calculations are unreliable.

To limit effects originating from the transverse motion from the $W$ Boson, we restrict ourselves to the study of $W$ Bosons with a small transverse momentum. Figure 2.6 displays the characteristic feature of the transverse mass; a slow rise with a peak at about the $W$ Boson mass, followed by a steep falling edge, (generally referred to as the "Jacobian edge"). 


\subsection{Measurement Strategy}

In this analysis, the transverse mass spectrum is used to extract the $W$ Boson mass. This spectrum cannot be predicted analytically. This is due to the limited detector acceptance, detector resolution and non-pertubative contributions to the transverse motion of the $W$ Boson at production. There are two important aspects in this analysis. Firstly, since the measurement of the $W$ Boson mass is a precision measurement, the detector has to be calibrated to the best possible level. Secondly, since the transverse mass spectrum cannot be predicted, a detailed simulation is needed which includes all relevant $W$ Boson production mechanism and detector effects. This section gives an overview of these two important aspects which will be explained in more detail in the remainder of this thesis.

The most important measured quantity which needs to be calibrated is the transverse momentum measurement of the muon candidate. It enters once directly into the transverse mass and a second time indirectly since the neutrino momentum is inferred by momentum conservation from the measured muon transverse momentum and the summed transverse momentum of the recoil particles. The muon transverse momentum can be precisely measured in the CDF magnetic spectrometer. The spectrometer can be calibrated by measuring particle masses which have been measured at other experiments with high precision. We use the quarkonium resonances $\Upsilon(1 S)$ and $J / \Psi$ for this task.

It is also for the inferred neutrino transverse momentum through momentum conservation that the recoil measurement effectively enters the transverse mass spectrum. The produced recoil balances the transverse momentum with which the $W$ Boson was produced. The recoil energy is measured in the calorimeter. In order to incorporate the recoil measurement into the simulation one is interested in how well the calorimeter measures the recoil for a given energy. A model for the recoil can be derived with the help of the $Z$ Boson. Since it has a similar mass compared to the $W$ Boson, it is ideally suited for this task. In $Z \rightarrow \mu \mu$ decays, the transverse momentum of both muon candidates can be measured relatively well in the magnetic spectrometer which allows for the 
computation of a well measured transverse momentum of the $Z$ Boson. This measured transverse momentum can be compared with the recoil energy measured in the calorimeter. This allows for a calorimeter response function to be derived which parametrizes the difference in the energy measurement of the recoil in the calorimeter compared to the transverse momentum of the $Z$ Boson measured in the spectrometer (which is close to the true transverse momentum of the $Z$ Boson).

All other relevant $W$ production mechanisms from theory predictions as well as detector effects have to be included in the simulation. This is realized by including NLO QCD and QED radiation in the production and decay of the $W$ Boson in the event generation. Important detector effects that are simulated are the tracking chamber and its acceptance and the material inside of it which causes particles to loose energy.

A transverse mass distribution can then be simulated which resembles the one from the data. To measure the $W$ Boson mass, different transverse mass distributions are simulated for a range of input masses in the form of templates. Figure 2.7 shows two extreme templates generated with input masses of 80 and $81 \mathrm{GeV} / \mathrm{c}^{2}$. In this analy-

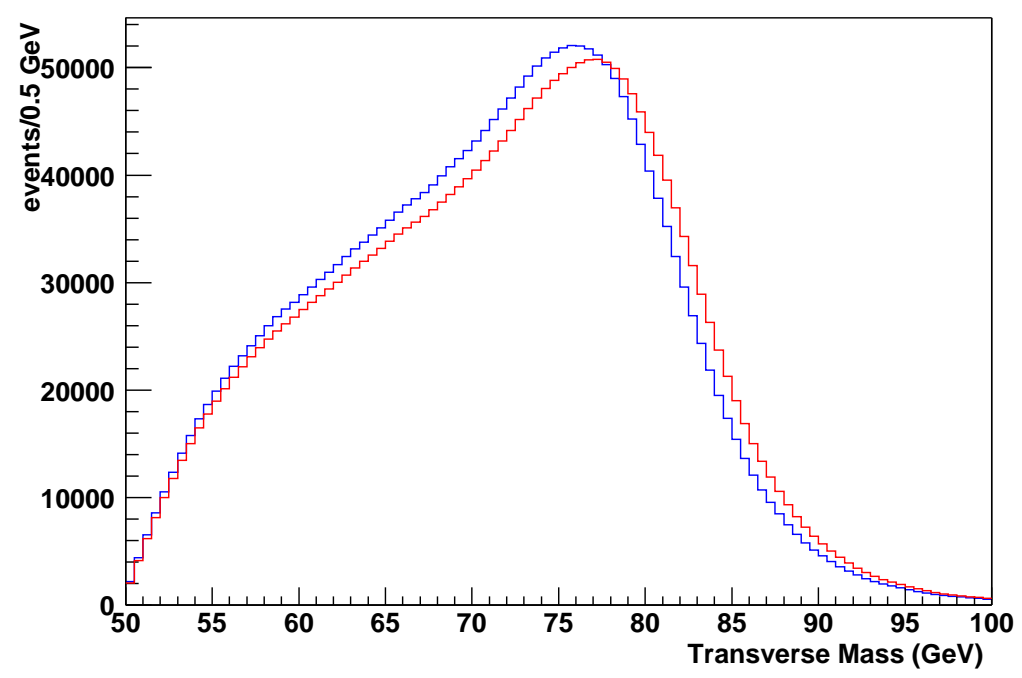

Figure 2.7: Transverse mass template for $80 \mathrm{GeV} / \mathrm{c}^{2}$ (blue histogram) and $81 \mathrm{GeV} / \mathrm{c}^{2}$ (red histogram). 
sis, eight hundred templates in between the two extreme input masses are generated. The measured backgrounds are added to the simulated templates and compared with the transverse mass distribution from the data. The template which compares best to the data distribution (through likelihood minimization) corresponds to the measured $W$ Boson mass. 


\section{Chapter 3}

\section{The Accelerator and Detector}

The Tevatron at Fermilab is the highest energy collider in the world. The Collider Detector at Fermilab (CDF) is located at a Tevatron collision point of proton and antiproton beams, carrying $980 \mathrm{GeV}$ of energy each providing a center of mass energy of $\sqrt{s}=1.960 \mathrm{TeV}[24]$. This chapter outlines the Tevatron accelerator complex and the CDF detector components important for this analysis.

\subsection{The Tevatron}

In Run 2 the increased center of mass energy of the Tevatron proton and antiproton collisions is $1.96 \mathrm{TeV}$. This corresponds to an increase of the $\mathrm{W}$ and $\mathrm{Z}$ production cross sections by about $14 \%$ [25] from the previous collider run (Run 1) where the center of mass energy was $1.8 \mathrm{TeV}$. The acceleration to the final $980 \mathrm{GeV}$ occurs in many stages in the Fermilab accelerator complex. Figure 3.1 shows the main components. In Run 2, the Tevatron also increased its luminosity. This was achieved by adding a new ring, the Main Injector, which greatly increased the rate of antiproton production and hence the number of antiprotons available for collisions. To keep the number of interactions per bunch crossing low, while at the same time raising the instantaneous luminosity, the number of proton and antiproton bunches in the ring were increased from 6 to 36 bunches. 


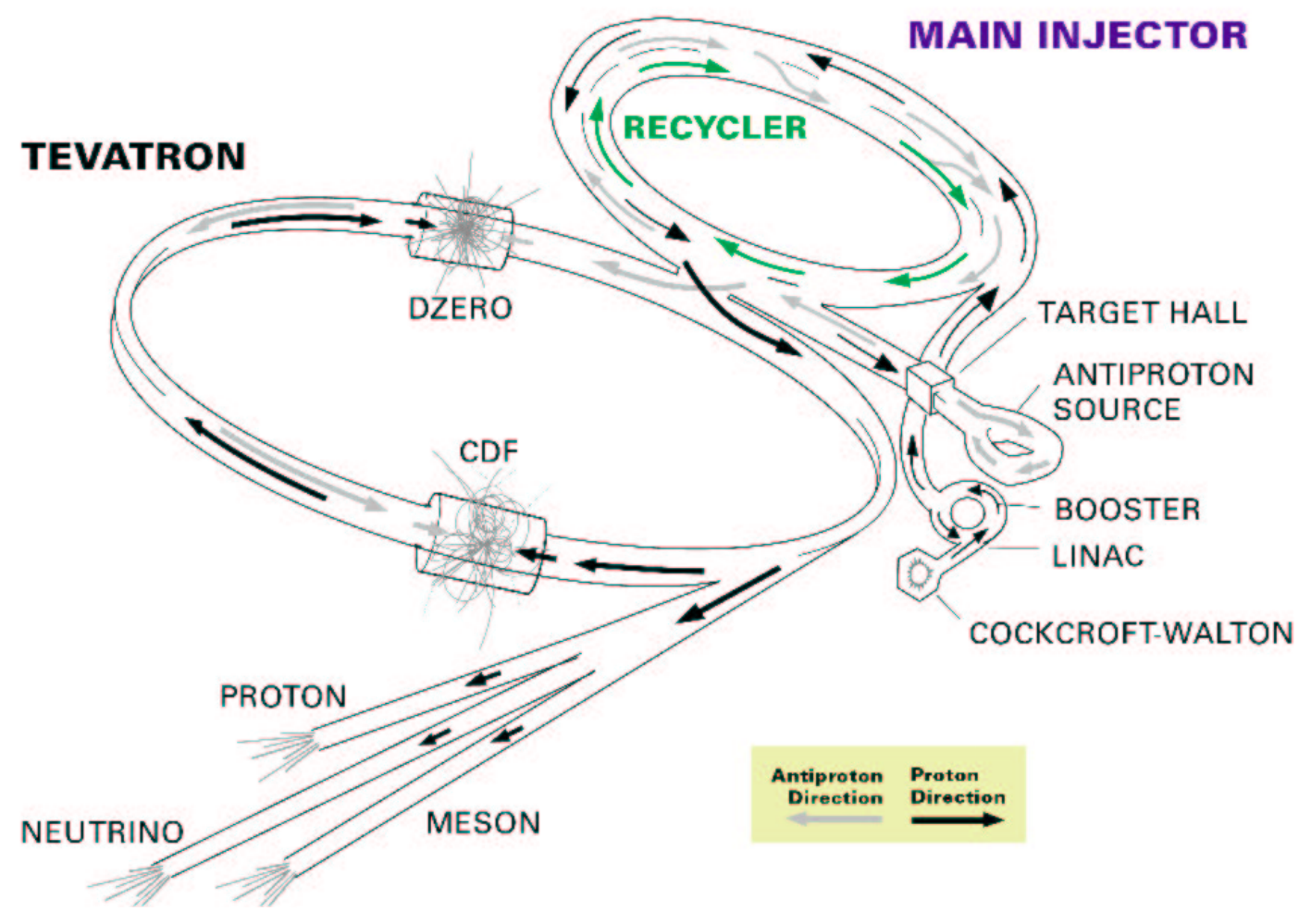

Figure 3.1: Schematic of the Tevatron accelerator chain.

This reduced the time between bunch crossings from $3.5 \mu$ s to 396 ns. A summary of the most important accelerator parameters of the Tevatron before and after the upgrades is shown in Table 3.1.

\section{Proton Source}

The starting point for the accelerator chain is the Cockcroft-Walton pre-accelerator which is a source of $750 \mathrm{keV}$ negative hydrogen ions. The $H^{-}$ions enter a $150 \mathrm{~m}$ long Linear Accelerator (Linac), that uses RF cavities to accelerate them to a final energy of 400 $\mathrm{MeV}$. After leaving the Linac, the ions enter into the Booster, the first synchrotron of the accelerator chain at Fermilab. During this transition, the $H^{-}$ions are passed through a carbon foil which strips off the electrons, leaving the bare protons to be accelerated. The Booster accelerates the protons to $8 \mathrm{GeV}$ in 84 bunches spaced by about $19 \mathrm{~ns}$. 


\begin{tabular}{lcc}
\hline \hline Accelerator Parameter & Run 1b & Run 2 \\
\hline$p \times \bar{p}$ bunches & $6 \times 6$ & $36 \times 36$ \\
Number of $p$ per bunch & $2.3 \times 10^{11}$ & $2.7 \times 10^{11}$ \\
Number of $\bar{p}$ per bunch & $5.5 \times 10^{10}$ & $3.5 \times 10^{10}$ \\
Beam energy $\left[\mathrm{GeV}^{10}\right.$ & 900 & 980 \\
Luminosity $\left[\mathrm{cm}^{-2} \mathrm{~s}^{-1}\right]$ & $1.6 \times 10^{31}$ & $8 \times 10^{31}$ \\
Bunch crossing time $[\mathrm{ns}]$ & 3564 & 396 \\
Number of interactions per crossing & 2.5 & 2.3 \\
$\bar{p}$ stacking & $6 \times 10^{10} / \mathrm{h}$ & $20 \times 10^{10} / \mathrm{h}$ \\
\hline \hline
\end{tabular}

Table 3.1: Accelerator parameters of the Tevatron.

\section{Main Injector}

The Main Injector is a synchrotron about $3 \mathrm{~km}$ in circumference that accepts protons from the Booster and continues the acceleration process up to an energy of $150 \mathrm{GeV}$. A majority of these protons are aimed at a nickel target inside the target hall to produce antiprotons. The Main Injector accelerates both protons and antiprotons from $8 \mathrm{GeV}$ to $150 \mathrm{GeV}$.

\section{Antiproton Production}

A major advantage of a $p \bar{p}$ collider, is that the $p$ and $\bar{p}$ beams can use the same magnet and vacuum system, while circulating in opposite directions resulting in collisions. The antiprotons emerging from the nickel target are collected, focused and separated from other by-products using a bending magnet which has a wide acceptance around $\bar{p}$ energies of $8 \mathrm{GeV}$. After this collection, the antiprotons are bunched. These antiprotons however have a large spread in momentum, which is reduced by a process called "stochastic cooling" [26], where the $\bar{p}$ beam is transformed into a continuous beam and cooled both transversely and longitudinally. In the Accumulator (not shown in Figure 3.1), antipro- 
tons are continually added in a process called "stacking". The stacking of antiprotons takes between half a day and a full day depending on the desired beam intensity. When a sufficient number of antiprotons are available, they are sent back to the Main Injector for further acceleration to $150 \mathrm{GeV}$.

\section{Tevatron}

The final stage of acceleration of the $p \bar{p}$ beams is the $6 \mathrm{~km}$ Tevatron ring. $150 \mathrm{GeV}$ protons and antiprotons are received from the Main Injector in 36 bunches and delivered to the Tevatron ring. Superconducting RF cavities and magnets are used to accelerate the protons to their final energy of $980 \mathrm{GeV}$.

\section{Collision Points}

The Tevatron has two dedicated collision points in the accelerator ring. One is being used by CDF, the other by another detector DØ. At the collision points, the Tevatron contains special focusing magnets to reduce the beam size to less than $30 \mu \mathrm{m}$. A typical colliding beam, called a store, lasts for approximately 15 hours. At the end of each store, the beams are aborted with a special beam dump. The Tevatron is then filled with new protons as well as the accumulated antiprotons to start a new store.

\subsection{The CDF Detector}

The data used for this measurement was collected with the upgraded Collider Detector at Fermilab (CDF II) [27]. Detector upgrades were made to accommodate the higher luminosities and new beam conditions. CDF is a general-purpose solenoidal detector that combines precision charged particle tracking with fast projective calorimetry and fine grained muon detection. A schematic view of the detector is shown in Figure 3.2. $\mathrm{CDF}$ is a cylindrically shaped apparatus with the axis of symmetry along the accelerator 


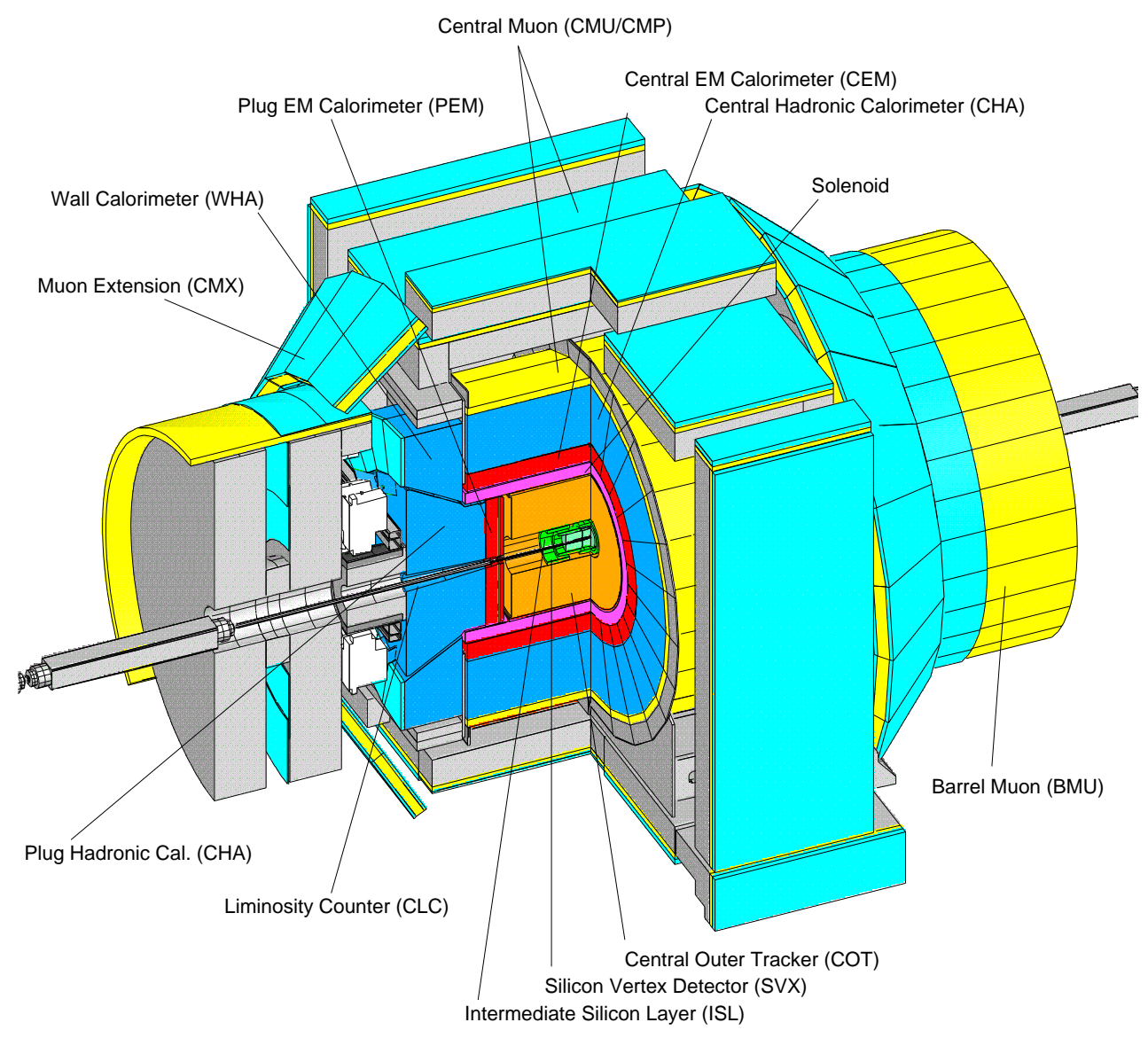

Figure 3.2: Schematic of the CDF detector.

beamline. It can be divided into three main subdetector components: a tracking system for particle momentum measurement, a calorimeter for energy measurement and a muon identification system. CDF uses a cylindrical coordinate system shown in Figure 3.3. The positive $z$-axis is defined along the direction of the proton beam. The radius $r$ is the distance from the beamline and the azimuthal angle $\phi$ is defined around the beam axis. The polar angle $\theta$, is measured relative to the incoming proton beam. It is often convenient to use the pseudo-rapidity $\eta(\eta=-\ln \tan (\theta / 2))$ instead of the polar angle $\theta$, since it transforms linearly under boosts in the $z$-direction ${ }^{1}$. The well instrumented central part of the detector spans a range of $|\eta| \leq 1$, while the forward part (plug) spans a range of $1.0 \leq|\eta| \leq 3.6$.

\footnotetext{
${ }^{1}$ This is only true in the limit $p \gg m$ when the pseudo-rapidity is equal to the rapidity $y=\frac{1}{2} \log \frac{E+p_{z}}{E-p_{z}}$.
} 


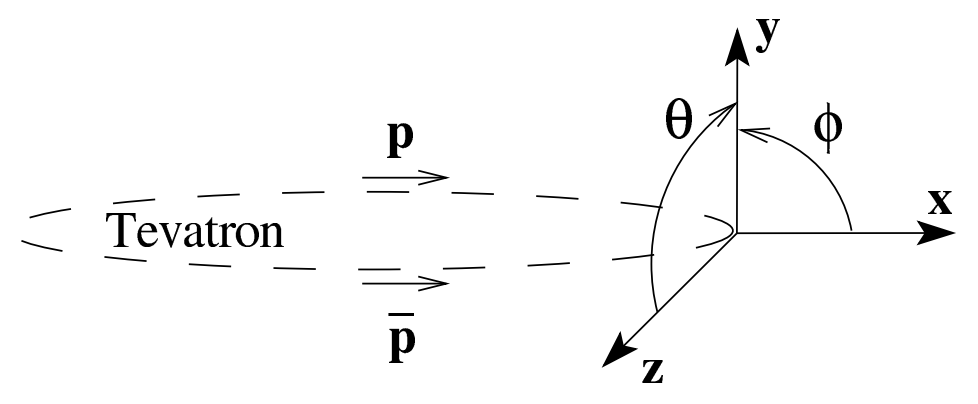

Figure 3.3: The CDF coordinate system.

\subsubsection{The Tracking System}

CDF is a powerful magnetic spectrometer which consists of several detector components as shown in Figure 3.4. The tracking system consists of a silicon micro-strip detector and an open-cell wire drift chamber that surrounds the silicon detector. The operation of tracking detectors is based on the fact that charged particles ionize the matter through

\section{CDF Tracking Volume}

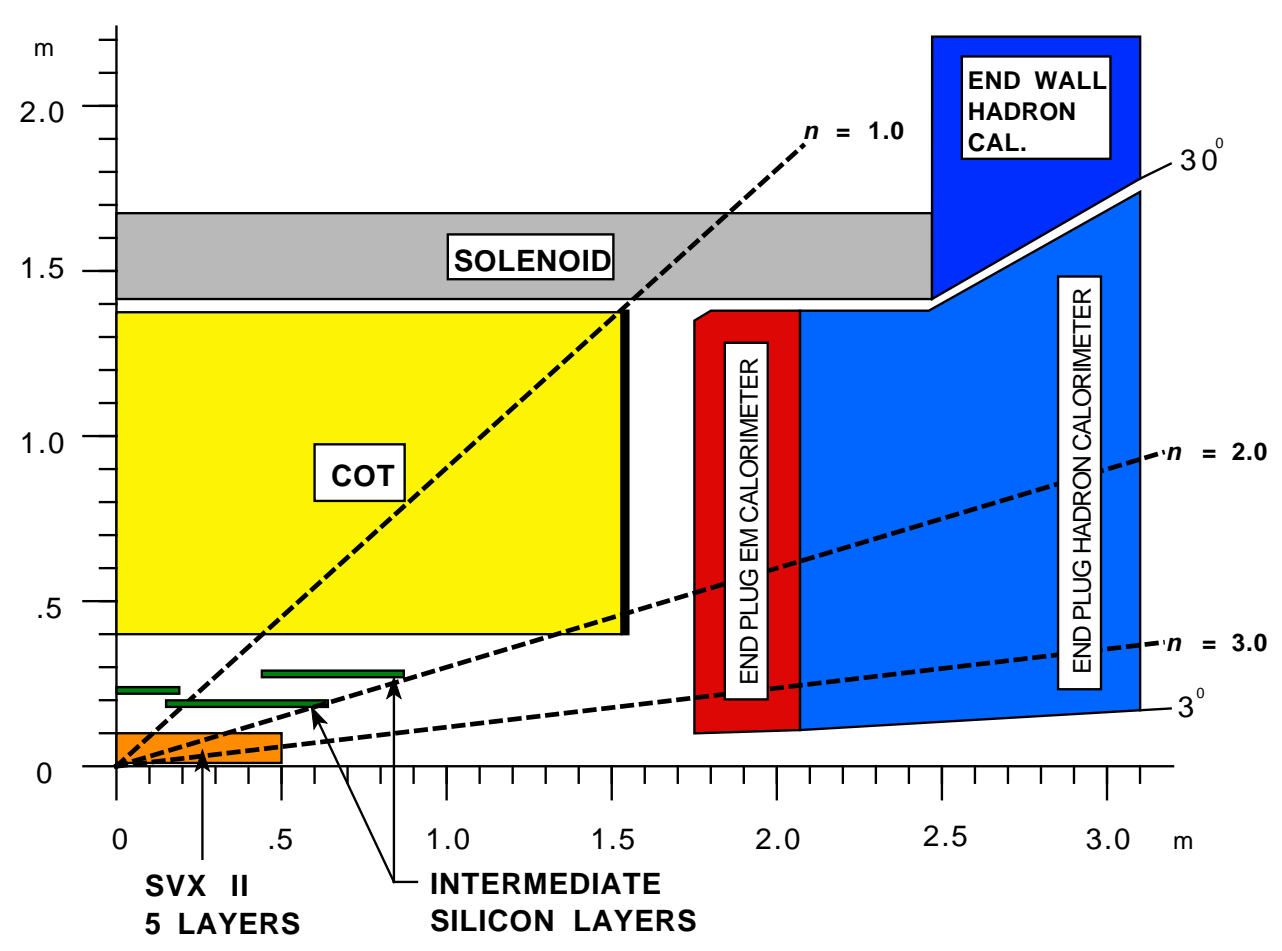

Figure 3.4: Schematic of the CDF tracking volume. 
which they pass. The trail of ions left behind by the charged particle coincides with its trajectory and is referred to as a track. The CDF magnetic field has a strength of $1.4 \mathrm{~T}$. It is provided by a $5 \mathrm{~m}$ long superconducting solenoid with coils operated at the liquid helium temperatures of about $4.7 \mathrm{~K}$. Charged particles moving in a uniform magnetic field have a helical trajectory. By measuring the radius of curvature of the helix, the particle's momentum can be measured.

\section{The Silicon Detector}

The silicon system of the tracking system has three parts. The first is a layer of radiation hard silicon wafers mounted directly on the beam pipe. The second sub-system is the Silicon VerteX detector (SVX II), consisting of five layers of double sided silicon wafers extending from a radius of 2.4 to $10.7 \mathrm{~cm}$ from the beam. The Intermediate Silicon Layer provides 2 more layers of double sided silicon wafers and extends from radii 20 to $28 \mathrm{~cm}$.

For this analysis we do not use the silicon detector for track reconstruction due to its limited angular coverage. But owing to its large mass, its material effect on the tracks of charged particles is important for this analysis in form of the energy loss it causes for the penetrating particles. This is significant especially for the low momentum tracks originating from the quarkonium resonances. For example a typical track from an $\Upsilon(1 S)$ decay with transverse momentum of $\sim 5 \mathrm{GeV} / \mathrm{c}$ looses $\sim 9 \mathrm{MeV}$ due to energy loss.

\section{The Central Outer Tracker}

Outside the silicon system is the upgraded open-cell drift chamber, called the Central Outer Tracker (COT). The drift chamber covers the radial region from 40 to $138 \mathrm{~cm}$ and is about $3 \mathrm{~m}$ long, which results in an $\eta$ coverage of $|\eta| \leq 1.0$ for tracks with hits in all layers. The main constituents of any drift chamber are wires that collect the ions produced by the charged particle in the gas contained in the tracking volume. The wires are grouped together into identical cells as shown in Figure 3.5. Within each cell, twelve 
sense wires are placed in an electrostatic field produced by seventeen potential and field shaping wires and two adjacent grounded field panels. The cells are grouped into eight superlayers. Axial superlayers, where the wires are parallel to the magnetic field alternate with stereo superlayers, where the wires have a $2^{\circ}$ stereo angle tilt. The innermost layer, which contains 168 cells, is a stereo layer. The outermost layer, an axial layer, contains 480 cells. The positions of all eight superlayers are shown in Figure 3.6.

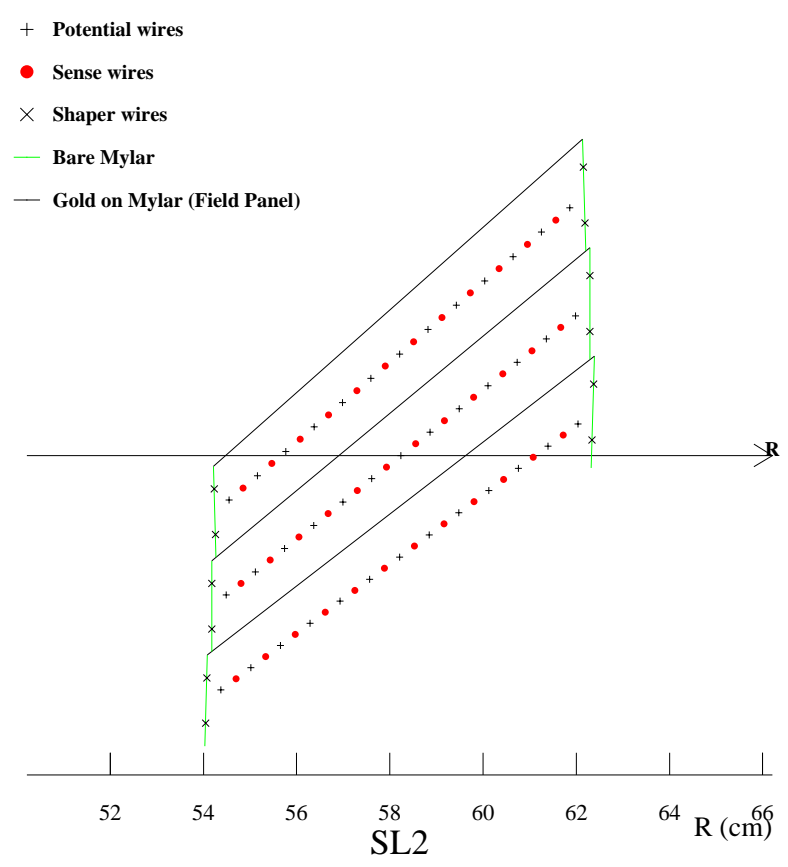

Figure 3.5: Cell configuration for superlayer 2 of the COT.

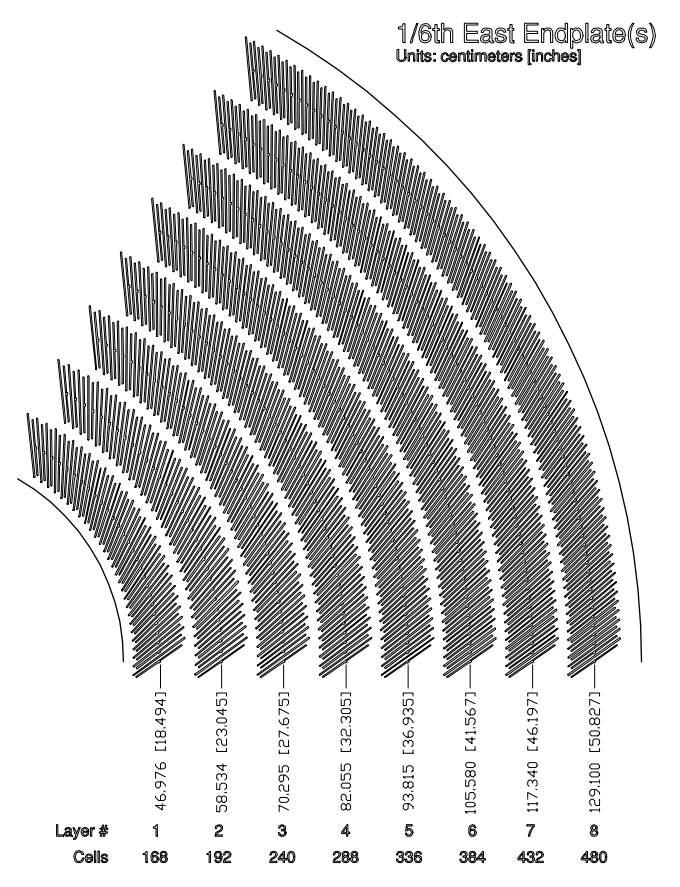

Figure 3.6: $1 / 6$ th view of the COT, including the radii $(\mathrm{cm})$ of each superlayer.

The most important design requirement for the COT was the ability to operate successfully at the high luminosity and collision rate of Run 2 . This requirement was met by choosing a small cell size and using a fast gas mixture. The COT uses the combination of Argon-Ethane- $\mathrm{CF}_{4}$ gases mixed in the ratio 50:35:15. The mixture provides a fast drift velocity on the order of $100 \mu \mathrm{m} / \mathrm{ns}$ at a drift field of $2.5 \mathrm{kV} / \mathrm{cm}$.

A dedicated tracking algorithm searches for a continuous pattern of localized charge depositions, which are referred to as hits, within a superlayer, then joins segments of hits to reconstruct the track of the charged particle. The transverse momentum of a 
reconstructed track is determined from $p_{T}=B q r$, where $B$ is the strength of the magnetic field, $q$ is the charge of the particle and $r$ is the radius of curvature. The curvature resolution is proportional to $1 / L^{2}$, where $L$ is the projected length of the track onto the bending plane. As a result, the resolution decreases for tracks with large $p_{T}$, which bend less in the magnetic field. The momentum resolution for COT tracks has been studied using incoming and outgoing "track legs" of reconstructed cosmic ray events and has been found to be $\sigma_{p T} / p_{T}^{2}=1.7 \times 10^{-3}[\mathrm{GeV} / \mathrm{c}]^{-1}$. The resolution of a track can be improved by measurements at large radial differences. This can be achieved by including track hits from the silicon detector or, for particles that originate from the interaction point, by including the position of the beamline as an additional hit (beam constrained tracks).

\subsubsection{The Calorimeter System}

Surrounding the tracking system and outside of the solenoid coil is the calorimeter. The calorimeter covers $|\eta|<3.6$. The presence of an energetic neutrino in an event is inferred through the transverse missing energy $\left(\mathbb{E}_{T}\right)$ calculated by taking the vectorial sum of transverse energies ${ }^{2}$ over all calorimeter units, called towers.

The CDF calorimeter is a scintillator sampling calorimeter, divided into separate electromagnetic and hadronic sections. The calorimeter is segmented into projective towers, with each tower consisting of alternating layers of passive material and scintillators. As particles pass through the calorimeter, they interact with the layers of material and produce "showers" of secondary particles. Light from the scintillators is read via wavelength shifters embedded in the scintillators or placed along the outside of the towers. The light from the wavelength shifters is further transmitted to photo-multiplier tubes where its intensity, proportional to the energy absorbed in the tower, is turned into an electrical signal which is then measured to determine the energy. The energy deposited in

\footnotetext{
${ }^{2} E_{T}=-\left|\sum_{i} E_{T}^{i} \hat{n}_{i}\right|$, where the $\hat{n}_{i}$ are unit vectors in the azimuthal plane that point from the $W$ Boson production point to the $i$ th calorimeter tower and $E_{T}=E \sin (\theta)$
} 
the calorimeter is summed together for each tower separately. The central barrel (and endwall part, see Figure 3.4) cover the pseudo-rapidity range $|\eta|<1.1$ (1.3). These sub-detectors are unchanged from Run 1 . The forward plug calorimeter $(1.1<|\eta|<3.6)$ has been upgraded for Run 2. The original gas calorimeter was replaced with scintillator plate calorimetry using the same polystyrene based scintillator and photomultiplier tubes as used in the central barrel.

The central barrel contains about 480 readout towers each covering $0.1 \times 15^{\circ}$ in $\eta \times \phi$ space. It is divided into two halves at $|\eta|=0$ consisting of 24 wedges in azimuth each. One wedge is shown in Figure 3.7. The energy resolution of the central electromagnetic calorimeter was measured in electron test beam data and was found to be $\sigma / E=14 \% / \sqrt{E_{T}}$.

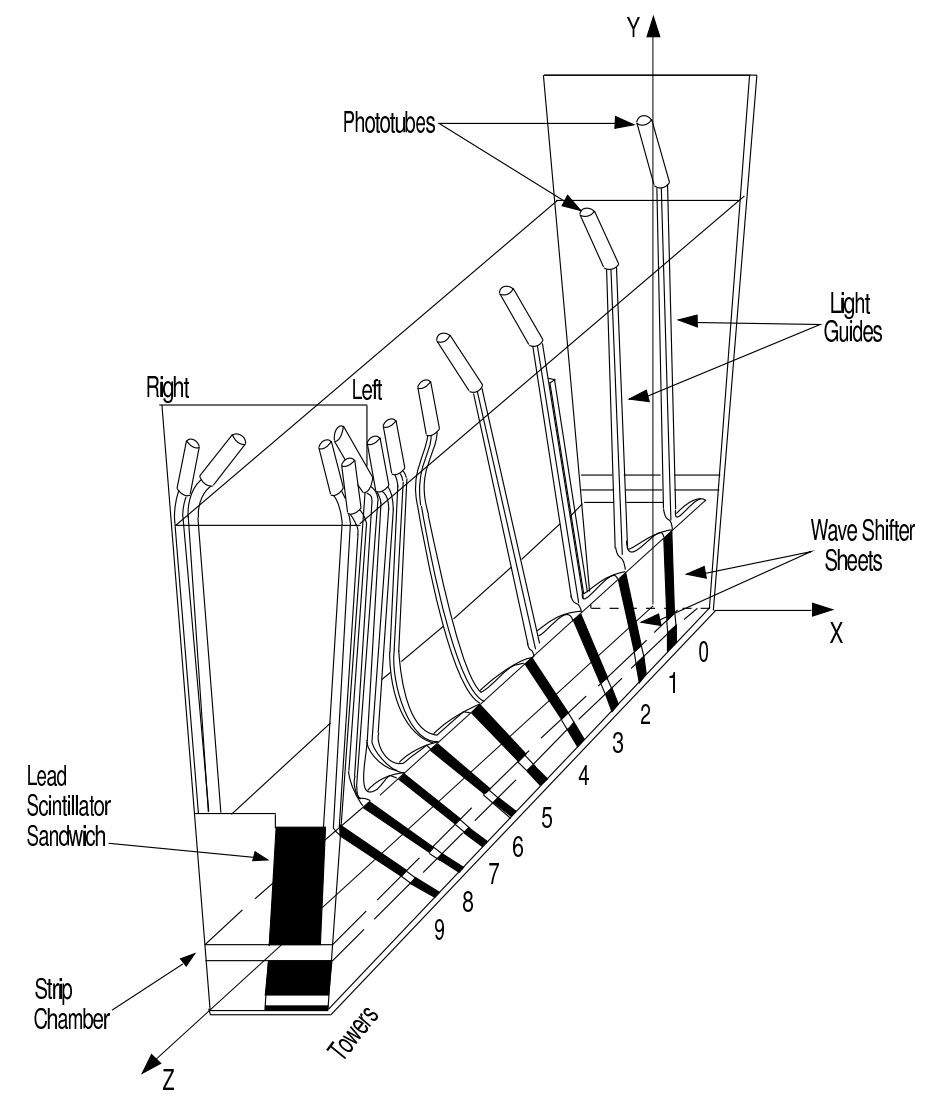

Figure 3.7: Schematic of a wedge in the central calorimeter. 


\subsubsection{The Muon System}

The outermost subsystem of the detector includes absorbers, scintillators, and stacks of drift chambers, used for muon identification. Due to their minimum ionizing behavior, muons can pass through a large amount of material, whereas the majority of other particles (apart from neutrinos) produced in the proton antiproton collision are absorbed by the calorimeter. The CDF muon system consists of four independent systems of proportional wire chambers and scintillators: the central muon detector (CMU) and the central muon upgrade (CMP), each covering $|\eta|<0.6$; the central muon extension (CMX) covering $0.6<|\eta|<1.0$; and the barrel muon detector (BMU) that covers $1.0<|\eta|<$ 1.5. We restrict ourselves to the well instrumented central part of the detector $|\eta|<1.0$ and thus do not use the BMU. The geometrical coverage of the central muon systems is shown as a $\eta-\phi$ map in Figure 3.8. The muon chambers are drift chambers filled with

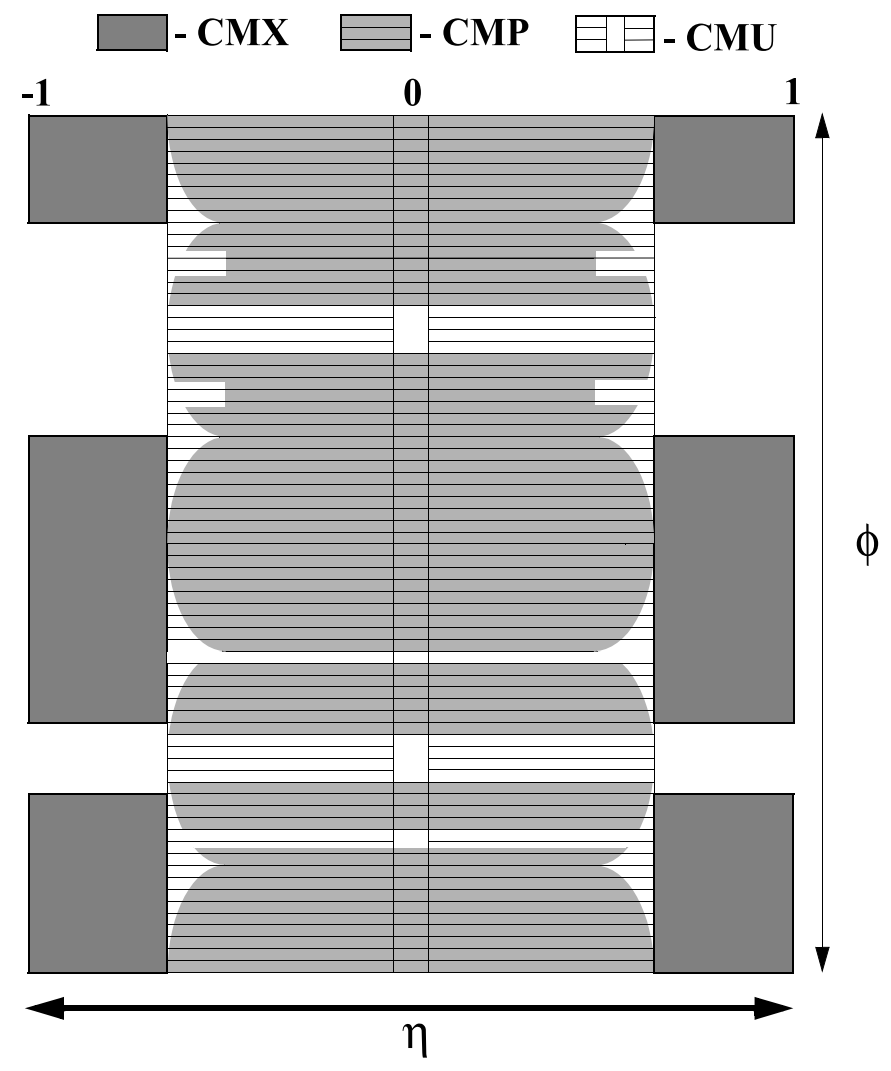

Figure 3.8: Coverage $(\eta, \phi)$ of the CDF central muon system. 
an Argon-Ethane gas mixture. When a muon candidate traverses the chamber, it ionizes the gas along its trajectory. The scintillator counters located outside the muon chambers provide additional evidence for the presence of a muon by generating a light pulse, which is collected by photo-multiplier tubes. The photomultiplier and scintillator are very fast and therefore can be used for triggering and to anchor the timing measurements obtained from the muon drift chambers.

\section{CMU}

The CMU is the original muon system built at CDF. It is located outside the hadron calorimeter at a radius of about $350 \mathrm{~cm}$ and has a cylindrically symmetric structure. With no additional absorber material between the calorimeter and the CMU, these chambers suffer from large backgrounds, originating from particles penetrating the hadron calorimeter. Figure 3.9 shows the location of one wedge of CMU chambers behind the central

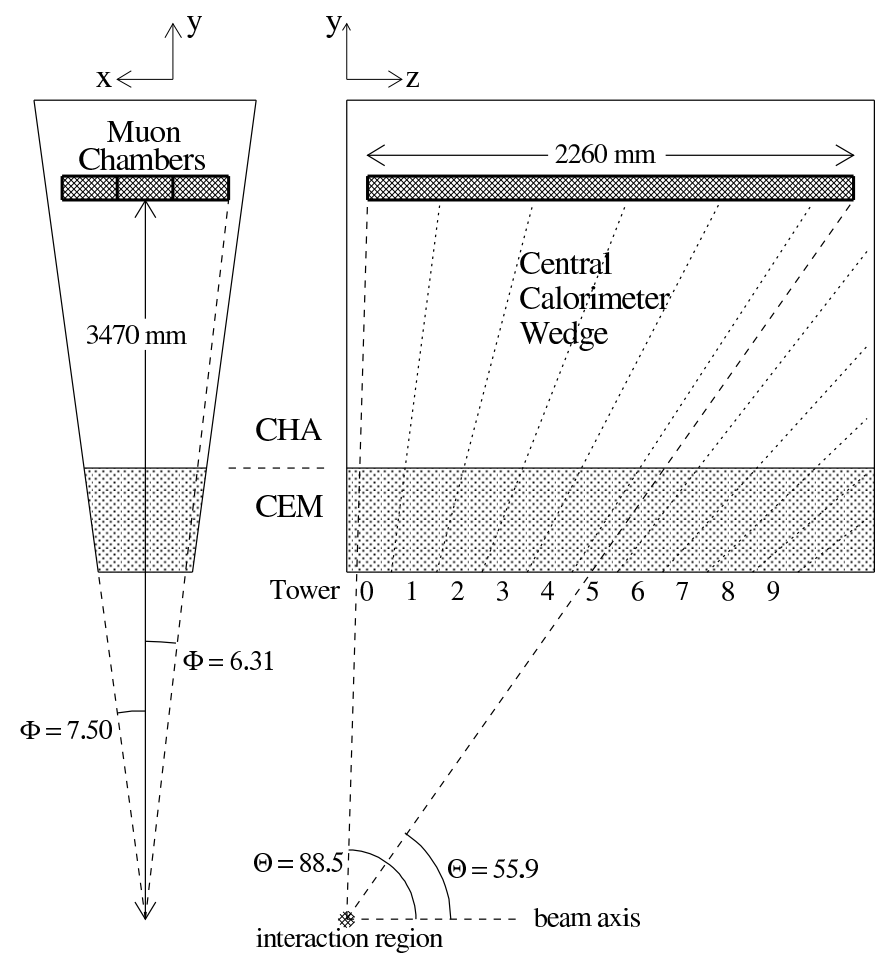

Figure 3.9: Schematic of one wedge of the CMU chambers. 
calorimeter. Each wedge covers $12.6^{\circ}$ in azimuth, whereas a calorimeter tower covers $15^{\circ}$, leaving a $2.4^{\circ}$ gap between the wedges. Each CMU wedge consists of three modules (stacks) with four layers of drift chambers as shown in Figure 3.10. The rectangular chambers have a $50 \mu \mathrm{m}$ sense wire at the center of the cell, oriented parallel to the $z$-axis.

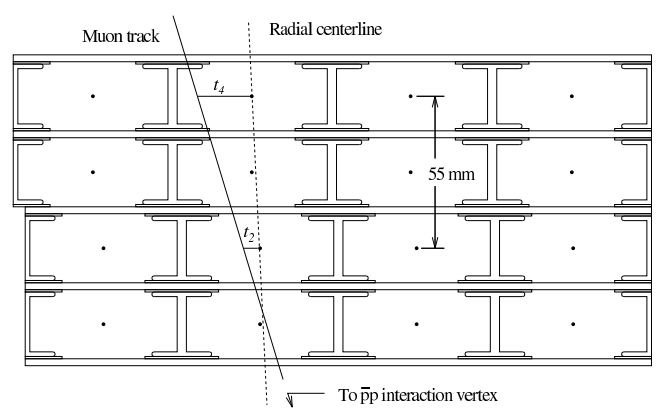

Figure 3.10: Transverse view of a CMU module.

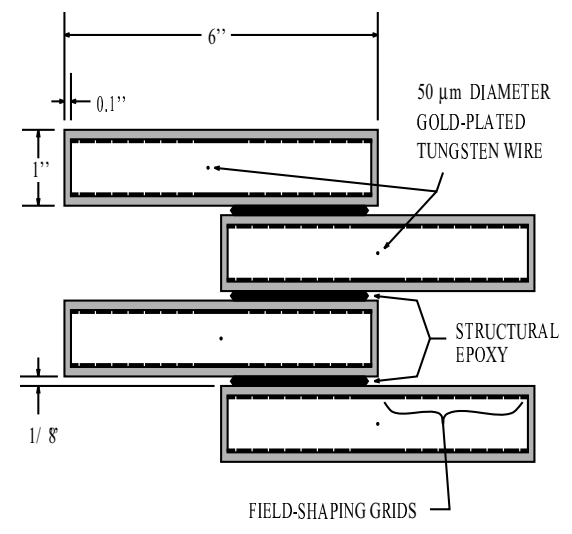

Figure 3.11: Transverse view of a CMP stack.

\section{CMP}

A second set of muon chambers, the CMP, is located behind an additional $60 \mathrm{~cm}$ of steel. This detector forms a square box around the CMU and its $\eta$ coverage therefore varies with azimuth as can be seen in Figure 3.8. The CMP consists of four layers of wire drift chambers of identical design to the $\mathrm{CMU}$, but they are staggered by a half cell per layer as shown in Figure 3.11. Located on top of the outermost layer of the CMP is the central scintillator upgrade (CSP), a layer of scintillator plates, to provide trigger and additional timing information.

\section{CMX}

The CMX consists of a conical arrangement of drift chambers which extends the pseudorapidity range to $|\eta|<1.0$. Though no additional absorbing material is added, the large 


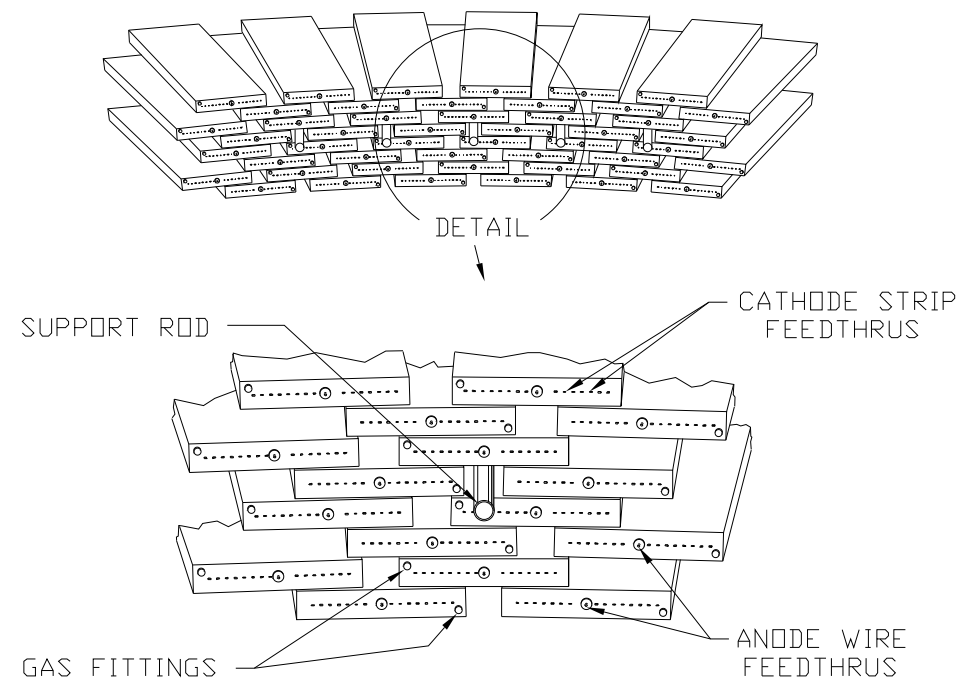

Figure 3.12: Drift chamber layout in a CMX module. The eight layers of chambers are grouped in pairs to form four continuous layers.

angle through the calorimeter and solenoid makes for more effective material along the muon path which provides shielding from backgrounds. Each section of CMX has $15^{\circ}$ of azimuthal coverage and has eight layers of rectangular drift chambers in the radial direction. The eight layers are grouped in pairs to form four continuous layers, each of which is stacked with a half-cell offset. The layout of the CMX is shown in Figure 3.12. A matching layer of scintillator plates (CSX) are mounted on both sides of the CMX system which is used in coincidence with the drift chambers.

\subsubsection{Luminosity Counters}

The beam luminosity is measured using low-mass gaseous Cherenkov luminosity counters (CLC). There are two CLC modules in the CDF detector installed at small angles in the proton and antiproton directions. Each module consists of 48 long, thin conical counters filled with isobutane gas and arranged in three concentric layers around the beam pipe. The CLC counters monitor the average number of inelastic $p \bar{p}$ interactions in each bunch crossing and are also used to trigger minimum bias events. 


\subsubsection{The Data Acquisition and Trigger System}

The increased collision frequency in Run 2 required the DAQ and "trigger system" of CDF to be replaced, although the basic architecture remained the same.

\section{The DAQ System}

The DAQ system is responsible for collecting data fragments from detector front-end electronic systems for events satisfying the trigger criteria, event building and subsequently sending them to mass storage. The front-end and trigger electronics are contained in VME modules that reside in about 120 crates in the system. Each crate contains at least one processor board for hardware initialization and event read-out plus additional boards for specific tasks.

\section{The Trigger System}

Collisions in the Tevatron occur at a rate of $2.5 \mathrm{MHz}$ and the average event size is about 250 KBytes/event. If one were to read out every event, one would have to write 630 GBytes/s to permanent storage. This is clearly too much to handle in a practical and economical way. Since not every event contains physics of interest, CDF utilizes a three level trigger system to select events for offline analysis. It is designed to provide sufficient rejection of uninteresting events to allow for processing with a minimal dead-time at each subsequent level. Figure 3.13 shows a functional block diagram of the CDF data flow.

The first decision and filtering level of CDF is the Level 1 trigger. It is a custom designed hardware system that selects events based on preliminary information from the calorimeter, tracking chamber and muon detectors. All detector data are fed into a 5.5 $\mu s$ pipeline to provide time for the processing required at Level 1. During that time, part of the data is examined in a first layer of dedicated, synchronous, highly parallel hardware processors. A tracking algorithm reconstructs tracks in the transverse plane using information from the four COT axial superlayers and an extrapolation unit matches 


\subsection{MHz Crossing rate}

132 ns Bunch Spacing (2.5 MHz / 396 ns)

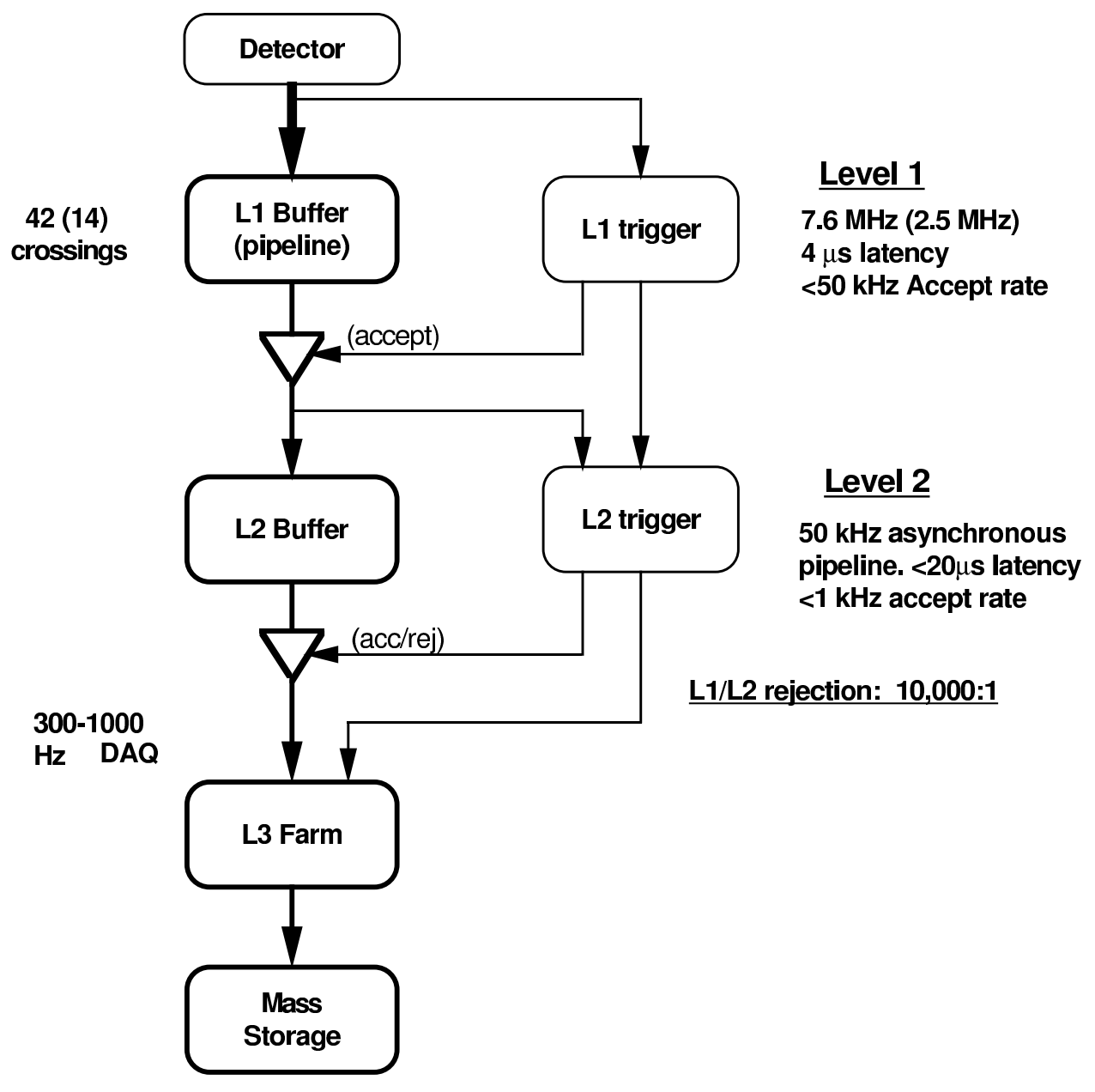

Figure 3.13: Diagram of the CDF dataflow (Rates quoted are design parameters).

these tracks to the Level 1 calorimeter and muon trigger boards. The calorimeter boards reconstruct electromagnetic and hadronic trigger energy depositions and calculate the total missing transverse energy in the event. The muon trigger boards match extrapolated tracks to hits in the the muon chambers. A total of sixty-four outputs from Level 1 sub-systems are sent to the global Level 1 Trigger board according to the configuration specified in the trigger database. The Level 1 trigger reduces the data rate from about $2.5 \mathrm{MHz}$ to less than $30 \mathrm{kHz}$.

The Level 2 trigger consists of a set of programmable logic devices that has access to 
more refined event information. The system has four asynchronous buffers, allowing the events to remain in the buffer system until accepted or rejected. The two phases of Level 2 include the readout of Level 2 systems and decision making on the event. Each phase lasts about $10 \mu \mathrm{s}$. During the decision-making stage, the readout data are fed to a pair of Alpha processors, with each processor examining the event for a different set of Level 2 trigger criteria as specified by the trigger data base. Level 2 provides a factor of 100 reduction, passing a $300 \mathrm{~Hz}$ data rate to Level 3 .

Events which pass Level 2 are analyzed by a Linux PC farm (Level 3), where they are completely reconstructed by the CDF offline software. Additional criteria are applied and events which pass this last trigger level are delivered to the data-logger system which transfers the data to the storage as well as to the monitoring system. Monitoring ensures that the detector and trigger system were working properly during data taking. A maximum rate of $20 \mathrm{MBytes} / \mathrm{s}$ can be written to mass storage. This corresponds to an event rate of about $80 \mathrm{~Hz}$ that can be accepted by Level 3 .

\subsubsection{Detector Operation}

The CDF detector is operated by a shift crew of five dedicated people plus several detector experts available on request. The crew operates in three eight hour cycles and consists of the detector Operations Manager, two ACEs who are responsible for common problems and the smooth running of the detector, one Consumer Operator who is trained to monitor and ensure good data quality, and one Scientific Coordinator who oversees the operations. At the end of each store, the shift crew labels the data good or bad and documents any special circumstances during data taking. This information is later used by the Data Quality and Monitoring group (DQM) to prepare a list of good runs usable for physics analyses (good run list). 


\subsubsection{Offline Data Reconstruction}

All data are reprocessed with the latest offline data analysis software before being used by the physics groups of the CDF Collaboration. The best information about detector alignment, calibration and reconstruction algorithm is used to prepare a common set of 'high level objects' such as electron, muon or track candidates. 


\section{Chapter 4}

\section{Data Sets and Event Selection}

Data samples from four different triggers are employed in this analysis to calibrate the detector. The $\mathrm{J} / \Psi \rightarrow \mu \mu$ and $\Upsilon(1 S) \rightarrow \mu \mu$ quarkonium decays are produced with large cross sections at the Tevatron. These two samples are used to calibrate the momentum scale of the tracker (see chapter 6 ). The $W \rightarrow \mu \nu$ and $Z \rightarrow \mu \mu$ candidates are contained in the same sample. Since the mass of the $Z$ Boson is of the same order of magnitude as the $W$ Boson it is very useful for this analysis. Compared to the $W$ Boson, where the neutrino does not interact with the detector, in $Z \rightarrow \mu \mu$ candidates both decay leptons are reconstructed, allowing the $Z$ Boson decays to be used to model the energy recoiling against the $W$ and $Z$ Bosons (see section 5.2.4 and chapter 8). The $Z$ Boson is also used as a cross-check of the momentum scale and to measure the tracking chamber hit resolution (see section 6.4). A minimum bias sample, collected at the same time as the $W$ and $Z$ samples is used to model the underlying event originating from spectator and multiple interactions occurring in the same beam-crossing as the $W$ and $Z$ candidates (see section 5.2.4 and chapter 8). 


\section{1 $W$ and $Z$ Sample}

$W$ Bosons decaying into a muon and a neutrino have a clear signature that consists of a muon with high transverse momentum and large missing transverse energy. We use the high- $p_{T}$ muon trigger path to select both $W$ and $Z$ boson events.

\subsubsection{High $p_{T}$ Muon Trigger}

Currently at CDF, there are two paths for high- $p_{T}$ muons: One with associated hits in both the CMU and CMP muon chambers (MUON_CMUP18 ${ }^{1}$ ) and the other with an associated hit in the CMX muon chamber (MUON_CMX18). From these triggers we obtain a raw dataset on which we then apply further analysis cuts to refine the data sample and reduce backgrounds. The muon trigger paths include the following requirements at the three trigger levels.

At Level 1, the muon trigger cards match tracks above $4 \mathrm{GeV} / \mathrm{c}$ to hits in the muon chambers. Hits are required in at least three of the four layers in the CMU or CMX chambers. A pattern of at least three CMP hits must match the hits in the CMU and be consistent in azimuth. For the CMX trigger, a matching hit pattern in the corresponding CSX scintillator counter has to be found. The matched hit pattern requirements are referred to as muon "stubs". Level 2 increases the track threshold to $p_{T}>8 \mathrm{GeV} / \mathrm{c}$.

At Level 3 the trigger code reconstructs a full COT track for the muon candidate and links it to the stubs in the muon chambers. At that point, the $p_{T}$ of the COT track must be larger than $18 \mathrm{GeV} / \mathrm{c}$. The code also checks the match between the extrapolated COT track trajectory and the muon stub in the plane transverse to the beam. The track must match the stub within $10 \mathrm{~cm}$ for $\mathrm{CMU}$ and $30 \mathrm{~cm}$ for CMP muon candidates. To be included in the sample, at least one muon candidate must meet all requirements. There is no explicit requirement on missing transverse energy in this trigger.

\footnotetext{
${ }^{1}$ This is the trigger name.
} 


\subsubsection{Candidate Selection}

The event selection criteria are designed to produce a sample low on backgrounds and with well understood muon and neutrino kinematics. The selection criteria need to be accurately modeled by the simulation. The $Z$ sample is used to derive a model for the energy recoiling against the $Z$ and $W$ Boson candidates and to measure the tracking resolution. In order to minimize biases in these measurements, the $Z \rightarrow \mu \mu$ selection is chosen to be as similar as possible to the $W \rightarrow \mu \nu$ selection. Candidate events have to satisfy criteria which we separate into muon identification requirements (listed in Table 4.1) and event selection requirements, listed in Table 4.2 for $W$ Boson and Table 4.3 for $Z$ Boson candidates. Muon candidates used in this measurement must have stubs in both the CMU and CMP chambers (CMUP muon) or a stub in the CMX chambers (CMX muon).

The muon candidate tracks have to extrapolate to well instrumented regions of the muon chambers. The position of the reconstructed chamber stubs are required to be close to the extrapolated track. The track-stub matching variable, $|\Delta x|$, is the distance between the extrapolated track and the muon stub in the $\mathrm{r}-\phi$ plane. When traversing the calorimeter the energy deposition in the electromagnetic, $E_{e m}$, and hadronic sections, $E_{\text {had }}$, of the calorimeters have to conform with the energy depositions expected from a minimum-ionizing particle. The associated reconstructed track of the muon candidate is required to originate within $60 \mathrm{~cm}$ in $z$ of the center of the detector and less than 0.2 $\mathrm{cm}$ away from the beamline in the $\mathrm{r}-\phi$ plane. We use tracks reconstructed solely in the COT that are constrained to the beamspot. At least five hits in three axial and three stereo superlayers have to contribute to the track which must traverse all of the eight superlayers. Finally a muon candidate must have transverse momentum, $p_{T}$, of at least $25 \mathrm{GeV} / \mathrm{c}$. For the $W$ sample we require one such muon candidate.

For the $Z$ sample, the first muon has to meet all the above criteria. The second muon is identified with a less restrictive criteria. The muon stub requirements are dropped but 


\begin{tabular}{lc}
\hline & Muon Candidate \\
\hline Geometric & Fiducial in CMUP or CMX \\
$|\Delta x|_{C M U}$ & $<3.0 \mathrm{~cm}$ \\
$|\Delta x|_{C M P}$ & $<5.0 \mathrm{~cm}$ \\
$|\Delta x|_{C M X}$ & $<6.0 \mathrm{~cm}$ \\
$E_{\text {em }}$ & $<2 \mathrm{GeV}$ \\
$E_{\text {had }}$ & $<6 \mathrm{GeV}$ \\
$p_{T}$ & $>25 \mathrm{GeV} / \mathrm{c},<55 \mathrm{GeV} / \mathrm{c}$ \\
$\#$ axial SL & $\geq 3 \mathrm{with} 5$ hits each \\
$\#$ stereo SL & $\geq 3 \mathrm{with} 5$ hits each \\
traversing all SL & \\
$\left|\Delta z_{0}\right|$ & $<60 \mathrm{~cm}$ \\
$\left|\Delta d_{0}\right|$ & $<0.2 \mathrm{~cm}$ \\
\hline
\end{tabular}

Table 4.1: Muon identification requirements.

the charge of the track must be the opposite sign of the first muon candidate and the track must have $p_{T}>25 \mathrm{GeV} / \mathrm{c}$.

For $W$ candidate events, the presence of the neutrino appears as a momentum imbalance in the event and the $E_{T}$ is required to be larger than $25 \mathrm{GeV} / \mathrm{c}$. For the $W$ selection, the recoil energy is required to be less than $20 \mathrm{GeV}$ which reduces QCD backgrounds and limits the transverse motion of the $W$ boson. For the $Z$ selection, the dilepton transverse momentum, determined from the two reconstructed muon tracks, is restricted to less than $30 \mathrm{GeV} / \mathrm{c}$. The cut on the $Z$ transverse momentum is chosen to be a factor of $50 \%$ larger than the recoil energy cut in the $W$ selection because the response of the calorimeter is, on average, $\sim 67 \%$ of the energy carried by the recoiling particles.

Energetic cosmic ray muons traverse the detector, depositing hits in both the muon chambers and the COT. Cosmic ray events are removed using a cosmic ray tagging 


\begin{tabular}{ll}
\hline \multicolumn{1}{c}{$W$ Event Selection } \\
\hline$E_{T}$ & $>25 \mathrm{GeV} / \mathrm{c},<55 \mathrm{GeV} / \mathrm{c}$ \\
$|U|$ & $<20 \mathrm{GeV}$ \\
not a cosmic candidate & \\
$Z$ rejection, no second track: & $p_{T}>10 \mathrm{GeV} / \mathrm{c}$ \\
& $E_{\text {em }}<2 \mathrm{GeV}$ \\
& $E_{\text {had }}<6 \mathrm{GeV}$ \\
& $i s o<0.11 / \mathrm{c}$ \\
& opposite charge \\
& $>50 \mathrm{GeV} / \mathrm{c}^{2},<100 / \mathrm{c}^{2} \mathrm{GeV}$ \\
\hline
\end{tabular}

Table 4.2: $W$ Boson candidate event requirements.

\begin{tabular}{l}
$\frac{Z \text { Event Selection }}{p_{T}^{\mu \mu}}<30 \mathrm{GeV} / \mathrm{c}$ \\
not a cosmic candidate \\
\hline
\end{tabular}

Table 4.3: $Z$ Boson candidate event requirements.

algorithm based on COT hit timing information [45] (see section 7.1). A large background to $W \rightarrow \mu \nu$ candidates comes from $Z \rightarrow \mu \mu$ events where one muon is not reconstructed. We reject events with a second minimum-ionizing track with transverse momentum above $10 \mathrm{GeV} / \mathrm{c}$ that is also isolated and has the opposite charge to the first muon candidate. The fractional isolation, iso, is defined as the calorimeter energy in a cone of radius $\Delta R=\sqrt{\Delta \eta^{2}+\Delta \phi^{2}} \leq 0.4$ around the muon, divided by the muon transverse momentum.

We also explicitly cut on the normalization regions, which we take as $100 \mathrm{GeV} / \mathrm{c}^{2}$ $>M_{T}>50 \mathrm{GeV} / \mathrm{c}^{2}$ for the transverse mass and $55 \mathrm{GeV} / \mathrm{c}>p_{T}>25 \mathrm{GeV} / \mathrm{c}$ for the transverse momentum and $55 \mathrm{GeV}>p_{T}>25 \mathrm{GeV}$ for the $E_{T}$. The normalization regions are related to the fit and will be explained later. This eliminates a large fraction of the decay in flight (Kaon) background (described in chapter 7), which would otherwise 
enter into the distributions used to tune the recoil model. Using the selection criteria described here, we find a total of $72418 W \rightarrow \mu \nu$ candidate events (with a purity of about $91 \%$, see chapter 7 ) and $5037 Z \rightarrow \mu \mu$ candidate events in $200 \mathrm{pb}^{-1}$ of data collected by CDF between 2001 and 2003.

\section{$4.2 \mathrm{~J} / \Psi$ and $\Upsilon$ Sample}

The $J / \Psi \rightarrow \mu \mu$ and $\Upsilon \rightarrow \mu \mu$ candidates are selected by a dimuon trigger.

\subsubsection{Dimuon Trigger}

At Level 1 tracks with transverse momentum above $1.5 \mathrm{GeV} / \mathrm{c}$ are selected. These tracks are passed to an extrapolation unit, where these trajectories are extrapolated to the muon chambers where stubs should be found if they are muons. The algorithm takes into account the path of the track in the magnetic field and computes the multiple scattering of a muon in the calorimeter. Two such tracks matched to hits in the muon system are required to pass the Level 1 dimuon trigger. There are two combinations possible. Either two CMU candidates with $p_{T}>1.5 \mathrm{GeV} / \mathrm{c}$ or a $1.5 \mathrm{GeV} / \mathrm{c} \mathrm{CMU}$ and a CMX candidate, where the CMX candidate has to have a $p_{T}>2 \mathrm{GeV} / \mathrm{c}$.

For the data presented here, no additional cuts were imposed at Level 2 for the $J / \Psi \rightarrow$ $\mu \mu$ trigger. For the $\Upsilon \rightarrow \mu \mu$, two trigger paths are possible. The UPSILON_CMUP_CMU trigger path asks for a CMUP candidate with $p_{T}>3.0 \mathrm{GeV} / \mathrm{c}$ in conjunction with a CMU candidate with $p_{T}>1.5 \mathrm{GeV} / \mathrm{c}$. The UPSILON_CMUP_CMX path requires a CMUP candidate with $p_{T}>3.0 \mathrm{GeV} / \mathrm{c}$ and a CMX candidate with $p_{T}>2.0 \mathrm{GeV} / \mathrm{c}$. At Level 3, the full reconstruction information is available. For the $\Upsilon$ sample, the $p_{T}$ cuts are further raised to $4.0 \mathrm{GeV} / \mathrm{c}$ for $\mathrm{CMUP}$ and $3.0 \mathrm{GeV} / \mathrm{c}$ for $\mathrm{CMU}$ and $\mathrm{CMX}$ candidates. Events with opposite charge muon pairs and dimuon masses between 2.7 and $4.0 \mathrm{GeV} / \mathrm{c}^{2}$ for the $\mathrm{J} / \Psi$ sample and 8.0 to $12.0 \mathrm{GeV} / \mathrm{c}^{2}$ for the $\Upsilon$ sample, are accepted. 


\subsubsection{Candidate Selection}

In addition to the trigger criteria, there are a few additional cuts imposed on the $J / \Psi$ and $\Upsilon$ samples listed in Table 4.4. The $p_{T}$ cuts are slightly raised relative to the trigger values to avoid trigger threshold biases. The requirement on the track hits are the same as for the $W$ and $Z$ samples. The impact parameter of the muon candidate track has

\begin{tabular}{lll}
\hline & \multicolumn{2}{c}{ Dimuon Candidates } \\
\hline Sample & $\mathrm{J} / \Psi$ & $\Upsilon$ \\
\hline$p_{T}$ & $>2.0 \mathrm{GeV} / \mathrm{c}$ & $>4.2 \mathrm{GeV} / \mathrm{c}(\mathrm{CMUP}),>3.2 \mathrm{GeV} / \mathrm{c}(\mathrm{CMU} / \mathrm{CMX})$ \\
$\#$ axial SL & 4 with 5 hits each & $\geq 3$ with 5 hits each \\
$\#$ stereo SL & 4 with 5 hits each & $\geq 3$ with 5 hits each \\
$\left|\Delta d_{0}\right|$ & & $<0.3 \mathrm{~cm}$ \\
$\left|\Delta z_{0}\right|$ & $<3.0 \mathrm{~cm}$ \\
\hline
\end{tabular}

Table 4.4: Dimuon candidate requirements for $\mathrm{J} / \Psi$ and $\Upsilon$ samples.

to be less than $0.3 \mathrm{~cm}$ and the $z$ position of the two muon candidate tracks must match within $3 \mathrm{~cm}$. Since $\mathrm{J} / \Psi \rightarrow \mu \mu$ candidates can originate from $B$ meson decays, we cannot constrain the tracks to the beamspot. Instead the tracks are reconstructed in the COT exclusively for this sample. The $\Upsilon \rightarrow \mu \mu$ candidates on the other hand, are produced promptly and can be reconstructed as COT tracks that are constrained to the beamspot (beam constrained tracks) and as COT only tracks (non beam constrained tracks). 


\section{Chapter 5}

\section{Simulation}

The transverse mass spectrum used to extract the $W$ Boson mass in this analysis cannot be predicted analytically. This is due to the limited detector acceptance, detector resolution and non-pertubative contributions to the transverse motion of the $W$ Boson at production. This measurement therefore needs a detailed, tunable and fast simulation that predicts the transverse mass spectrum for a given true mass. The CDF collaboration uses a standard detector simulation (cdfSim) based on GEANT [28][29]. This simulation is rather slow $(<1 / 2$ events/s) and somewhat difficult to tune. As a result, a fast detector simulation was developed for this measurement, several aspects of which were derived from the standard simulation where the simulated effects were parametrized. The fast Monte Carlo model consists of two parts. First we simulate the production of the particles and their decay products by generating the particle four-vectors. These generated events are then input into the fast detector simulation, which produces simulated templates as a function of generated mass used to measure different masses. The fast detector simulation can produce transverse mass templates for $W \rightarrow \mu \nu$ events and invariant mass templates for $J / \Psi \rightarrow \mu \mu, \Upsilon \rightarrow \mu \mu$ and $Z \rightarrow \mu \mu$ events. 


\subsection{Event Generation}

Two different Monte Carlo event generation schemes are used for the two qualitatively different particle samples: quarkonium resonances and intermediate vector Bosons. Since we measure only the transverse mass for the $W$ Boson, it is important to use a $\mathrm{NLO}^{1}$ event generator to properly model gluon radiation off the annihilating quarks as this residual energy feeds into the neutrino $p_{T}$. Another aspect of concern is QED radiation, because it introduces $\mathrm{O}\left(100 \mathrm{MeV} / \mathrm{c}^{2}\right)$ corrections to the generated $W$ and $Z$ Boson lineshapes. To simulate these corrections, we use a NLO QED event generation. For the quarkonium resonances, we measure the invariant mass and QED effects are significantly smaller than for the $\mathrm{W} / \mathrm{Z}$ Bosons.

\subsection{1 $J / \Psi$ and $\Upsilon$ Generation}

For the quarkonium resonances: $J / \Psi$ and $\Upsilon$; we use the PYTHIA event generator to simulate the kinematics of the event. Since PYTHIA does not simulate QED radiation for quarkonium resonances, we simulate internal muon bremsstrahlung within the fast simulation according to a Sudakov form factor [30]:

$$
f_{\mu}^{\mu}(x)=\beta(1-x)^{\beta-1}
$$

where

$$
\beta=\frac{\alpha_{e m}}{\pi}\left(2 \cdot \ln \frac{m_{J / \Psi, \Upsilon}}{m_{\mu}}-1\right)
$$

$f_{\mu}^{\mu}(x)$ is the $\mu \rightarrow \mu$ fragmentation probability and $x$ is the energy fraction retained by the muon. The curvature of the simulated muon track is reduced according to the energy fraction simulated by the internal bremsstrahlung.

The PYTHIA output kinematics are tuned in two ways in order to better match the data.

\footnotetext{
${ }^{1}$ next-to-leading order
} 


\section{Boost to $p_{T}^{\mu \mu}$}

We tune the $p_{T}^{\mu \mu}$ distribution by increasing the decay muons' rapidity in the direction of the $p_{T}^{\mu \mu}$ boost. The fit to the invariant mass is sensitive to this distribution. Furthermore, a good match in the $p_{T}^{\mu \mu}$ distribution between data and simulation provides a similar statistical representation of higher and lower momentum tracks. Figure 5.1 shows the $p_{T}^{J / \Psi}$ distribution as generated by PYTHIA. Figure 5.2 shows the $p_{T}^{J / \Psi}$ distribution after the tuning is applied. For all data - Monte Carlo comparisons, the data is represented by blue points while the Monte Carlo is shown by a red histogram. The data is sideband subtracted using $3.01 \mathrm{GeV} / \mathrm{c}^{2}<m^{\mu \mu}<3.15 \mathrm{GeV} / \mathrm{c}^{2}$ as the signal region and $3.17 \mathrm{GeV}$ $<m^{\mu \mu}<3.31 \mathrm{GeV}$ as the sideband region.

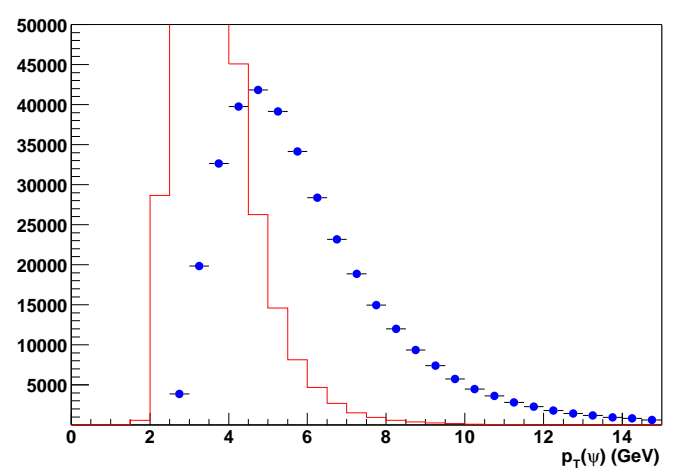

Figure 5.1: The $p_{T}^{J / \Psi}$ distribution straight out of PYTHIA.

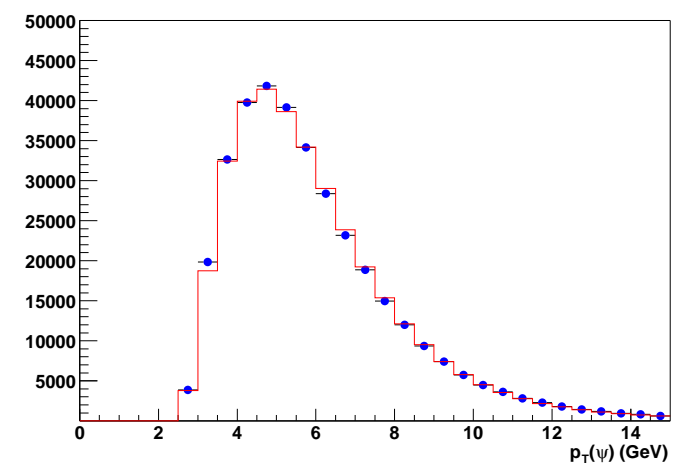

Figure 5.2: The $p_{T}^{J / \Psi}$ distribution after tuning.

\section{Change of the Muon Decay Angle $\theta^{*}$}

We change the muon decay angle $\theta^{*}$ by scaling $\cot \theta^{*}$ in the rest frame of the decaying $J / \Psi$ meson, shown in Figure 5.3. This increases the fraction of decays in the direction of the $p_{T}^{\mu \mu}$ boost, which increases the asymmetry of the curvature of the two decay muons in each event. This is important, since the momentum scale is examined as a function of the average curvature of the two decay muons (see next chapter). Figure 5.4 shows the sum of 


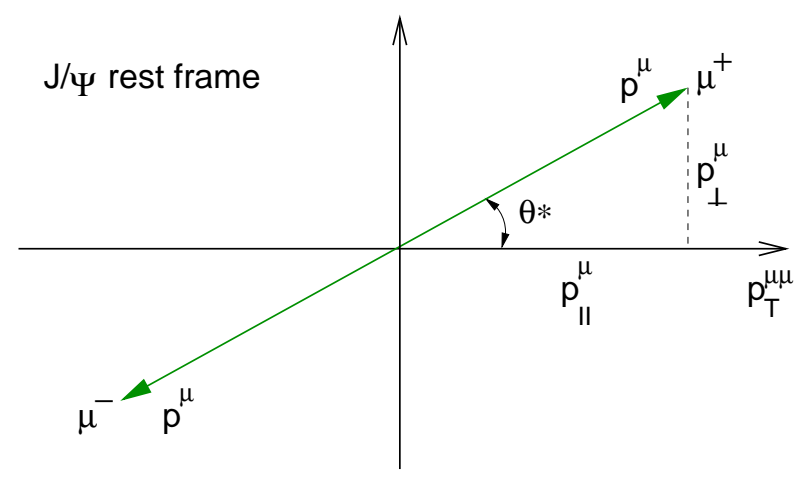

Figure 5.3: Kinematics in the quarkonium rest frame.

the curvature of the two decay muons. Figure 5.5 shows the same distribution after the $\theta^{*}$ tuning is applied. After the two adjustments are applied, we find good agreement between

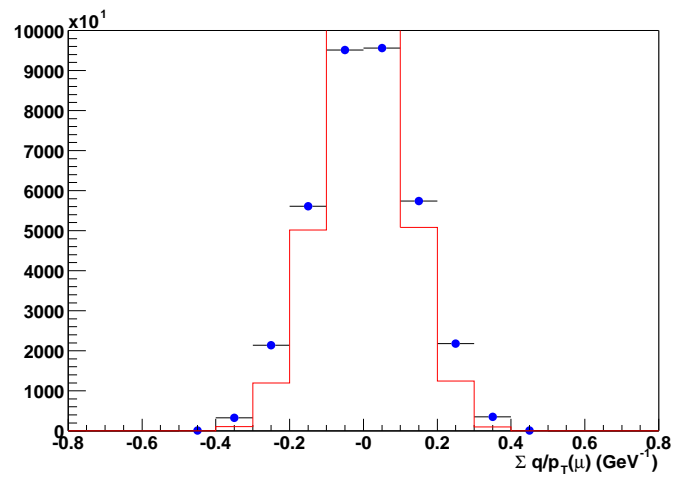

Figure 5.4: The sum of the curvature distribution straight out of PYTHIA.

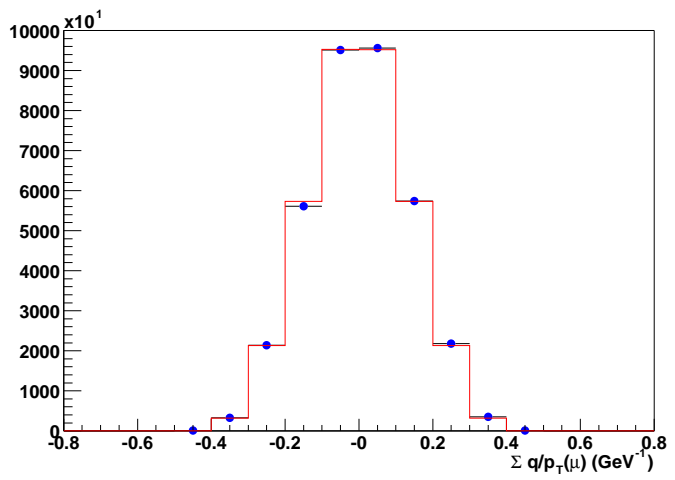

Figure 5.5: The sum of the curvature distribution after $\theta^{*}$ tuning is applied.

data and simulation for all the distributions that have been examined. These include the muon candidate transverse momentum, the average curvature, angular distributions and momentum distributions of the $J / \Psi$ and muon candidate in the $z$ direction.

\subsection{2 $W$ and $Z$ Generation}

The two important aspects of the $W$ and $Z$ Boson event generation which are described here arise from QCD and QED effects. 


\section{QCD}

We generate $W$ and $Z$ events with RESBOS [31], which is a NLO QCD event generator. RESBOS computes the quintuple differential cross-section

$$
\frac{d^{5} \sigma}{d Q_{T} d y d Q^{2} d \Omega}
$$

for $p \bar{p} \rightarrow W / Z$ and models the transverse momentum spectrum of the $W$ and $Z$ Bosons well at low $p_{T}^{W / Z}$ via multiple soft gluon resummation techniques. The production crosssection is given by the partonic cross-section convoluted with the parton distribution functions. The non-pertubative physics at low $p_{T}^{W / Z}$ is parametrized by three parameters $g_{1}, g_{2}$ and $g_{3}$, which must be determined experimentally. The dominant effects on the $p_{T}^{W / Z}$ spectrum arise from changes in $g_{2}$. Figure 5.6 shows the $p_{T}^{W}$ spectrum for three different values of $g_{2}$. We use the following $g$ parameters in RESBOS, obtained from a

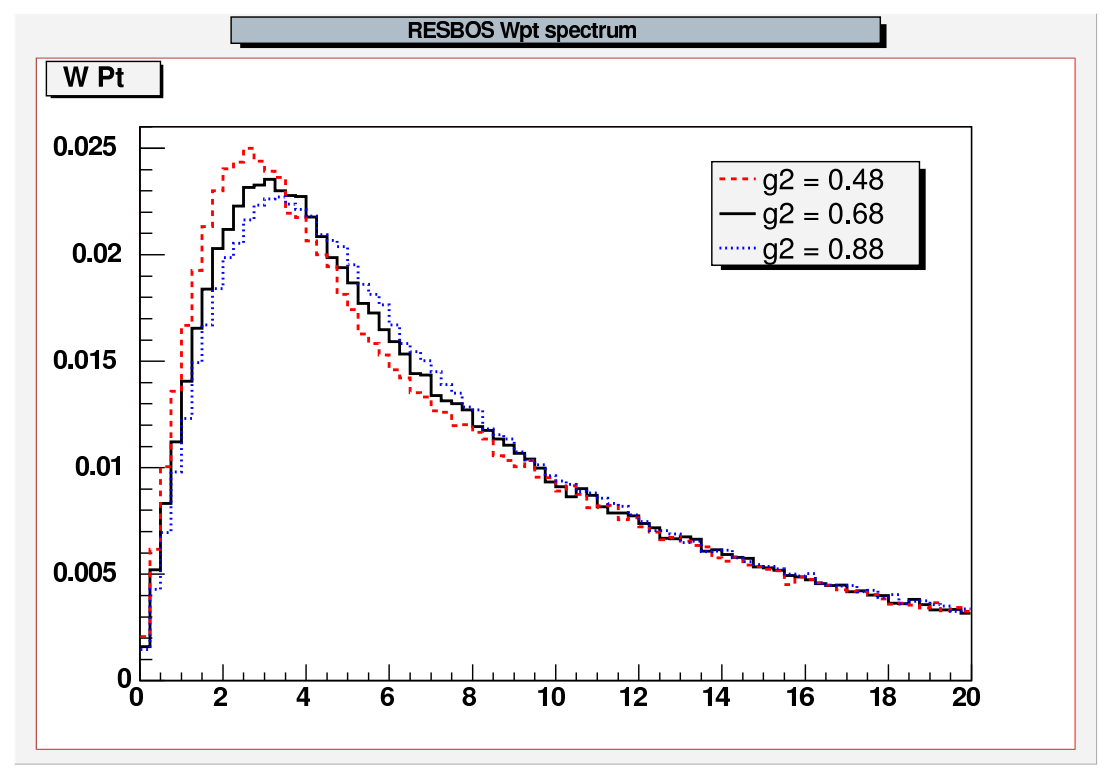

Figure 5.6: $p_{T}^{W}$ spectrum generated by RESBOS for various $g_{2}$ values. The mean $W p_{T}$ increases as $g_{2}$ increases.

global fit to low-energy Drell-Yan and Tevatron Run $1 Z$ Boson data [32].

$$
g_{1}=(0.21 \pm 0.12) \mathrm{GeV}^{2}
$$




$$
\begin{gathered}
g_{2}=(0.68 \pm 0.12) \mathrm{GeV}^{2} ; \\
g_{3}=(-0.60 \pm 0.3) .
\end{gathered}
$$

The uncertainties listed, are the variations we use to estimated the systematic uncertainty on the $W$ Boson mass. To estimate the systematic uncertainty from the $p_{T}^{W}$ model, we simulate $W$ Boson transverse mass spectra varying the $g_{2}$ and $g_{3}$ parameters by their uncertainties. We vary $g_{2}$ by \pm 0.12 and leave $g_{1}$ fixed. This variation agrees with [33], that also shows that $g_{1}$ and $g_{2}$ are $100 \%$ anti-correlated. Thus, varying $g_{2}$ by \pm 0.12 [34] is equivalent to varying $g_{1}$ by a similar amount. In addition, following [33] we vary $g_{3}$ by \pm 0.3 . The systematic uncertainties obtained from these variations are $7 \mathrm{MeV} / \mathrm{c}^{2}$ from $g_{2}$ and $11 \mathrm{MeV} / \mathrm{c}^{2}$ from $g_{3}$. Combined with the $4 \mathrm{MeV} / \mathrm{c}^{2}$ statistical uncertainty from the fits, we obtain an overall $14 \mathrm{MeV} / \mathrm{c}^{2}$ systematic uncertainty on the $W$ Boson mass from the $\mathrm{QCD} / p_{T}^{W}$ production model.

\section{QED}

The QED corrections to $W$ and $Z$ production and decay are simulated with WGRAD [35], that calculates all $\mathrm{O}(\alpha)$ electroweak processes including a real single final-state photon as well as the final state soft and virtual photon corrections. We incorporate the WGRAD physics by adding WGRAD photons to the two-body simulated RESBOS events [36]. Various QED radiation scenarios are investigated by simulating corresponding transverse mass data distributions and templates. The dominant effect on the measured $W$ Boson mass is a single photon radiated off the final state muon. When not included in the simulation, the $W$ Boson mass is underestimated by $149 \pm 5 \mathrm{MeV} / \mathrm{c}^{2}$. Photon radiation off the incoming quarks and the $W$ propagator were investigated but no statistically significant effect was observed. We therefore take the $5 \mathrm{MeV} / \mathrm{c}^{2}$ statistical uncertainty as a systematic and include only final state photon radiation from WGRAD in our simulation.

We capture the physics of the final state photons from WGRAD in a two dimensional histogram of $\sqrt[3]{k}$ versus $\sqrt{\Delta R}$, where $k=E_{\gamma} /\left(E_{\gamma}+E_{\mu}\right)$ and $\Delta R(\mu, \gamma)=\sqrt{(\Delta \eta)^{2}+(\Delta \phi)^{2}}$. 
The cube and square-roots flatten the sharply peaked distributions of $k$ and $\Delta R$, respectively, and make the sampling of the distributions in a Monte Carlo simulation more efficient. Figure 5.7 and 5.8 show the projections of these two variables. To save com-

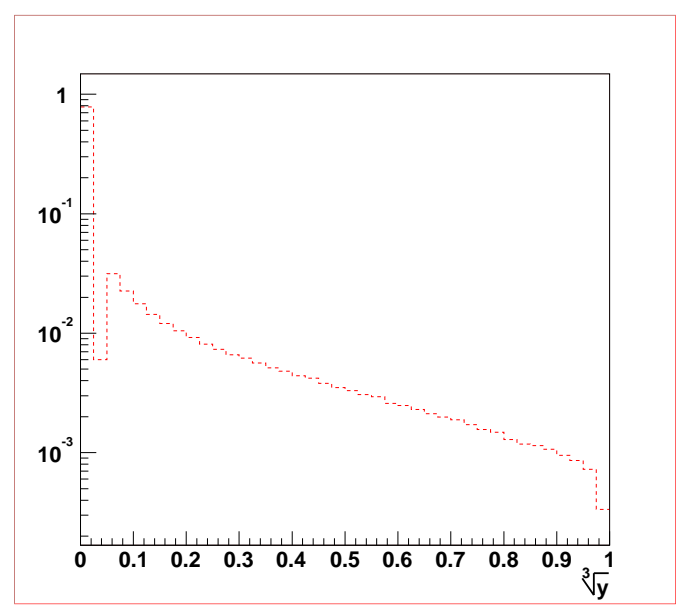

Figure 5.7: Projection of $\sqrt[3]{k}$. Events with no photons are in the zero bin.

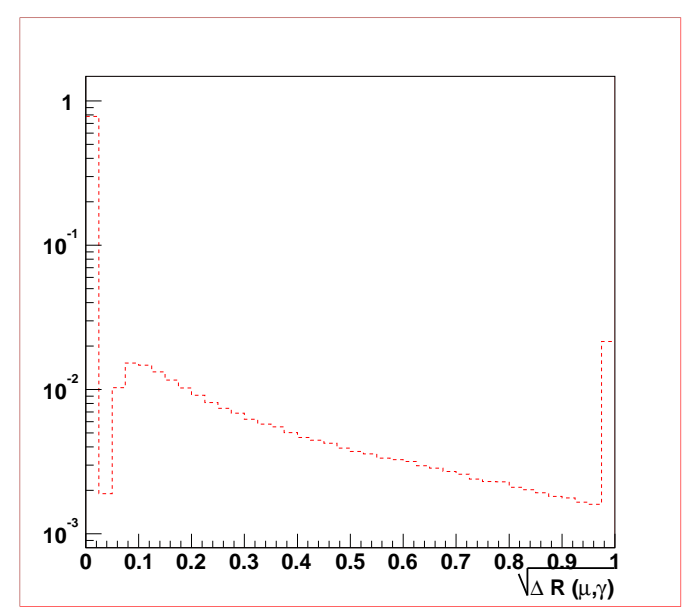

Figure 5.8: Projection of $\sqrt{\Delta R(\mu, \gamma)}$. Events with $\Delta R>1$ are in the last bin.

putation time, we only simulate photons above an energy threshold of $\sim 4 \mathrm{MeV}$. The intrinsic WGRAD parameter is called $\delta_{S}$, and controls the photon energy cut off

$$
E_{\gamma}=\delta_{S} \sqrt{s} / 2
$$

where $\sqrt{s} / 2$ is the parton energy in the parton-parton center of mass. To investigate the systematic uncertainty on this variable, we vary $\delta_{S}$ over an extreme range and take its variation as a systematic uncertainty. No statistical significant variation is observed and we take the $5 \mathrm{MeV} / \mathrm{c}^{2}$ statistical uncertainty as a systematic. Additional QED uncertainties arise from multi-photon radiation which has not been included in the simulation. We take $10 \%$ of the single final state photon shift as a systematic uncertainty for this short coming [37]. Table 5.1 summarizes the systematic uncertainties on the $W$ Boson mass arising from QED. 


\begin{tabular}{lc}
\hline \hline Source & Systematic $(\mathrm{MeV})$ \\
\hline ISR/Interference & 5 \\
$\delta_{S}$ & 5 \\
Second Photon & 15 \\
\hline Total QED & 20 \\
\hline \hline
\end{tabular}

Table 5.1: Summary of QED systematics on the $W$ Boson mass measurement.

\section{Proton Parton Densities}

Parton distribution functions enter into the $W$ Boson mass measurement through their effects on the detector acceptance and kinematics of the decay charged lepton. The PDF constraints are obtained from global fits to many data sets such as deep inelastic electronproton scattering, jet production and $W$ Boson charge asymmetry. There are two major groups that provide semi-regular updates to parton distributions when new data or theoretical calculations become available, namely the CTEQ and MRS collaborations. The CTEQ6 PDF [38] allows twenty free parameters in a global fit to the data. It uses a Hessian method, where a twenty dimensional matrix is diagonalized and twenty orthogonal eigenvector directions in parameter space are determined. Each eigenvector direction corresponds to a linear combination of PDF parameters. From these eigenvectors, forty uncertainty PDFs are constructed, covering $90 \%$ of the probability of PDF space. In order to obtain the variation due to the forty uncertainty PDFs, events are generated in PYTHIA for each PDF employing an interface to the PDFs in RoOT [39]. A Monte Carlo template generated with the central CTEQ6 PDF (CTEQ6M) is then used to obtain the best fit for each uncertainty PDF. After simulating the detector acceptance and response, we obtain the variation of the forty CTEQ6 error PDFs compared to the fit result of the CTEQ6M PDF (Figure 5.9). The error bars indicate the statistical uncertainty from the fits. In order to estimate the PDF systematic uncertainty on a given measured quantity, the CTEQ collaboration provides a formula to compute the uncertainty corresponding 


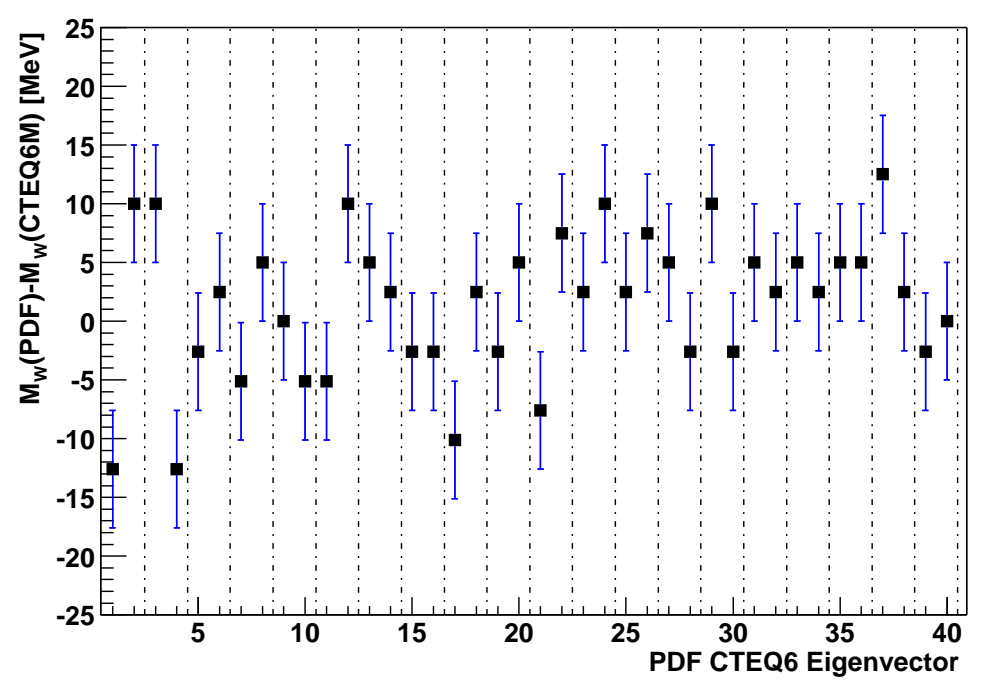

Figure 5.9: Variation of the twenty CTEQ6 pdf error pairs.

to the $90 \%$ coverage. To obtain a $1 \sigma$ systematic uncertainty on the $W$ Boson mass, we divide this formula by a factor of 1.6 :

$$
\text { CTEQ6 ' } 1 \sigma^{\prime} \text { ' uncertainty }=\frac{1}{2} \sqrt{\sum_{i}\left(\Delta M_{W}(+)_{i}-\Delta M_{W}(-)_{i}\right)^{2}} / 1.6=15 \mathrm{MeV} / \mathrm{c}^{2} \text {, }
$$

where $\Delta M_{W}( \pm)_{i}$ corresponds to the measured mass difference between the CTEQ6M template and the PDF data with the varied uncertainty, for a given eigenvector $i$. The factor $1 / 2$ appears because the differences $\Delta M_{W}$ are computed for both, the positive and negative direction of PDF space. The factor 1.6 normalizes the uncertainty to a $1 \sigma$ equivalent. The latest PDF from the MRS collaboration [40] is found to fit well within the CTEQ6 error band. Generating forty million events using the MRST2003 PDF and fitting the obtained transverse mass distribution with the CTEQ6M template, we find an $8 \mathrm{MeV} / \mathrm{c}^{2}$ difference with respect to the CTEQ6M fit. This falls well into the $15 \mathrm{MeV} / \mathrm{c}^{2}$ uncertainty found by the CTEQ6 method. A systematic uncertainty of $15 \mathrm{MeV} / \mathrm{c}^{2}$ on the $W$ Boson mass for the $M_{T}$ fit due to the PDF choice is therefore adopted. 


\subsection{Fast Detector Simulation}

The fast detector simulation captures all relevant detector effects. It simulates the tracker, the calorimeter response, the passive material in the detector and the muon acceptance. At its heart is a fast hit-level tracking simulation. The simulation reads in vector files containing three-momentum and vertex information as produced from the event generators (described above).

\subsubsection{Simulation of Passive Material}

The effect of passive material is important for muons with low momentum originating from $J / \Psi \rightarrow \mu \mu$ and $\Upsilon \rightarrow \mu \mu$ events, which have (after selection cuts) average transverse momenta of $\sim 3 \mathrm{GeV} / \mathrm{c}$ and $\sim 5 \mathrm{GeV} / \mathrm{c}$ respectively. The two main effects modeled are ionization energy loss and multiple coulomb scattering. The passive material of the COT inner support structure and the silicon vertex detector (SVXII) is represented by a three-dimensional look-up table called "silimap" [41]. Silimap was created by scanning the full detector geometry as implemented in the standard CDF GEANT simulation (cdfSim). The implementation in cdfSim is based on material measurements during detector construction and was tuned using electrons from photon conversions in Run 2 data. Silimap stores all material properties as a function of radius, azimuth and $z$ in a finely binned three-dimensional look-up table implemented as a three-dimensional histogram. It stores three material properties, namely, the number of radiation lengths, $X_{0}$, needed to evaluate the multiple scattering and the normalization and ionization constants needed to evaluate the Bethe-Bloch formula for ionization energy loss [42]:

$$
-\frac{d E}{d x}=K z^{2} \frac{Z}{A} \frac{1}{\beta^{2}}\left[\frac{1}{2} \ln \frac{2 m_{e} c^{2} \beta^{2} \gamma^{2} T_{\max }}{I^{2}}-\beta^{2}-\frac{\delta}{2}\right]
$$

where $A$ and $Z$ are the atomic mass and atomic number of the absorber, $z$ is the charge of the incident particle, $\beta$ is the velocity of the incident particle, $m_{e}$ is the electron mass, $\gamma$ the ratio of particle energy to its mass, $T_{\max }$ is the maximum kinetic energy which can 
be imparted to a free electron in a single collision and $\delta$ is the density effect correction to ionization energy loss. The mean excitation energy $I$ (ionization constant) and $K Z / A$ (normalization constant) are the two parameters obtained from silimap.

A charged particle traversing material is deflected by many small-angle scatters. Most of this deflection is due to multiple Coulomb scattering from the nuclei. For multiple scattering, the radiation length is used to evaluate a first order representation of a fit to a Moliere distribution which describes Coulomb scattering:

$$
\theta_{0}=\frac{13.6 \mathrm{MeV}}{\beta c p} z \sqrt{x / X_{0}}
$$

where $p, \beta c$ and $z$ are the momentum, velocity and charge of the incident particle and $x / X_{0}$ is the thickness of the scattering medium in radiation lengths. $\theta_{0}$ is the average small-angle scatter a muon would be expected to undergo.

\subsubsection{Tracker Simulation}

The COT tracks associated with the muons are simulated using a hit-level simulation. The muon is propagated along a helical trajectory from the production point, stepping through the passive material described by silimap. At the intersection with each layer of silimap, the number of radiation lengths $X_{0}(r, \phi, z)$ is used to deflect the particle according to the most probable small-angle Coulomb scattering. The particle energy is reduced by the ionization energy loss predicted by the Bethe-Bloch formula. The COT wires are simulated at the appropriate radii and stereo angles. The hit and superlayer efficiencies are tuned to match the measured data tracks. The COT hits are smeared according to a Gaussian distribution with a resolution measured from the data. The smeared hits are fit with a helix to obtain the five track parameters curvature $(c)$, azimuth $\left(\phi_{0}\right)$, polar angle $(\theta)$, impact parameter $\left(d_{0}\right)$ and track vertex $\left(z_{0}\right)$. Depending on the ionization energy loss the muon experienced, the helix parameters curvature, $\phi_{0}$ and $d_{0}$, are updated accordingly. The fitted track parameters are used to compute the track 
kinematics, such as the transverse momentum. The simulation has also the option to beam constrain the fitted track, where the $p \bar{p}$ collision point is included in the track fit.

\subsubsection{Muon Acceptance Modeling}

The acceptance of the muon detectors is extracted from the standard CDF simulation, cdfSim. Single muons with a transverse momentum of $40 \mathrm{GeV} / \mathrm{c}$ and a flat distribution in azimuth and $\eta$ are generated with a simple one particle event generator. These muons are propagated through the detector and reconstructed with the CDF offline code. The probability of identifying a muon in the CMUP and CMX detectors is evaluated as a function of azimuth $\phi_{0}, \cot \theta$ and $z_{0}$. Figure 5.10 shows the probability of finding a CMUP muon as a function of $z_{0}$ and $\cot \theta$ as well as the probability of finding a CMX muon as a function of $\phi_{0}$ and $\cot \theta$. The variation with $z_{0}$ is small over the luminous region of the beam and so this dependence is neglected. Little correlation is observed between the $\phi_{0}$ and $\cot \theta$ dependence and therefore we do not simulate one. Figure 5.10b shows the uninstrumented regions of the CMX in the $\phi$ region 1.4 to 1.8 and 4.2 to 5.4 . Since there is little correlation, we create four one dimensional look-up tables to evaluate the probability to find a CMUP or a CMX muon as a function of $\phi$ and $\cot \theta$. In order to match the ratio of CMUP and CMX muon $W$ candidates observed in the data, we

a)

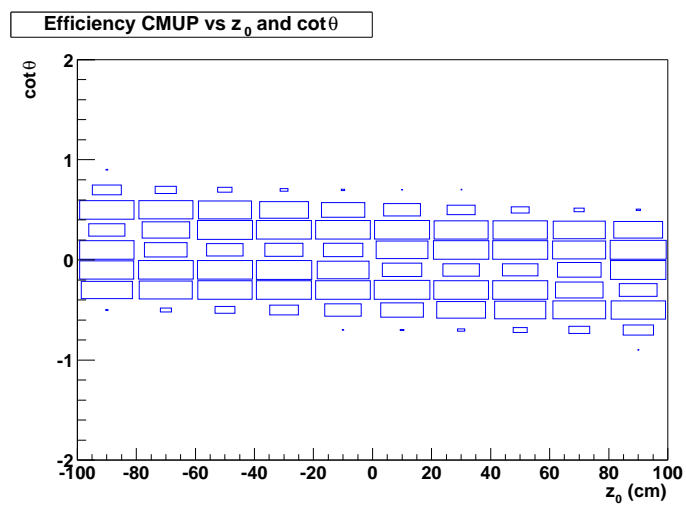

b)

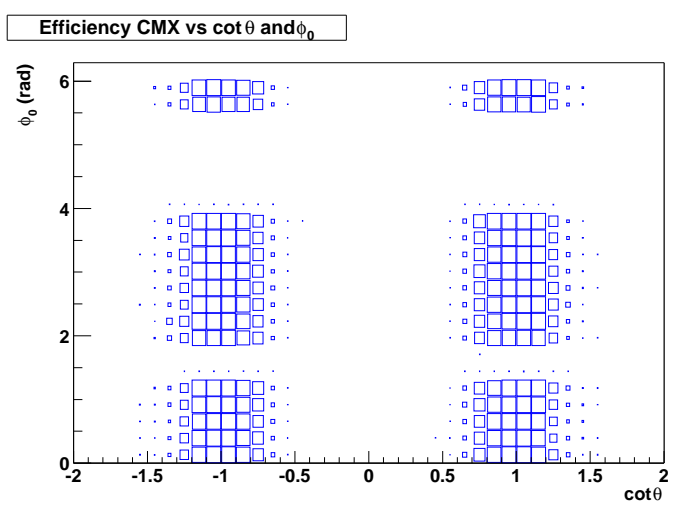

Figure 5.10: a) Probability of finding a CMUP muon as a function of $z_{0}$ and $\cot \theta$ and b) probability of finding a CMX muon as a function of $\phi_{0}$ and $\cot \theta$. 
renormalize the probabilities with one overall scale factor for the two CMUP acceptance maps. The muon identification probability is then evaluated in the fast simulation using the relation:

$$
\epsilon^{\mu}(\phi, \cot \theta)=\epsilon_{\phi}^{C M U P}(\phi) \cdot \epsilon_{\cot \theta}^{C M U P}(\cot \theta)+\epsilon_{\phi}^{C M X}(\phi) \cdot \epsilon_{\cot \theta}^{C M X}(\cot \theta)
$$

In addition to the simulated acceptance of the muon detectors, cuts are made to represent the fiduciality of the COT. The comparison between the data and simulated $W \rightarrow \mu \nu$ events are shown in Figure 5.11 for the $\cot \theta$ and $\phi_{0}$ distributions.

\subsubsection{Calorimeter Response}

All particles recoiling against the $W$ or $Z$ Bosons are referred to as the recoil. The recoil vector, $\mathbf{u}$, is calculated by summing over all electromagnetic and hadronic calorimeter towers within the detector range $|\eta|<3.6$

$$
\mathbf{u}=\left(u_{x}, u_{y}\right)=\left(\Sigma_{\text {towers }} E \sin \theta \cos \phi, \Sigma_{\text {towers }} E \sin \theta \sin \phi\right) .
$$

There are two contributions to the recoil. The first is the energy of the initial state gluons radiated from the quarks that produce the $W$ or $Z$ Boson. This energy balances the transverse momentum of the Boson. The second is the energy associated with the remnants of the protons and antiprotons that are involved in the $W$ or $Z$ production

a)

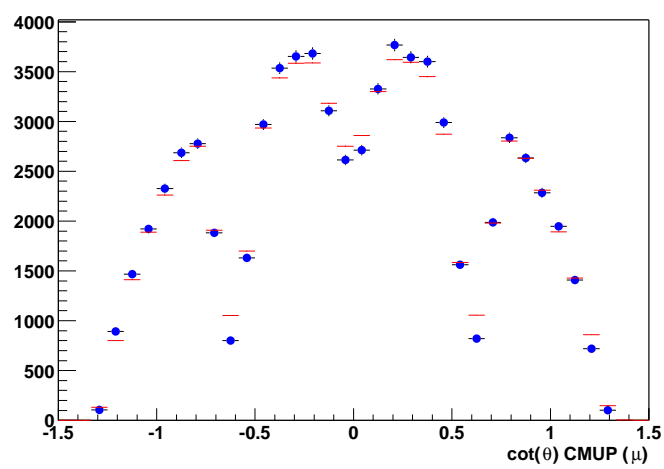

b)

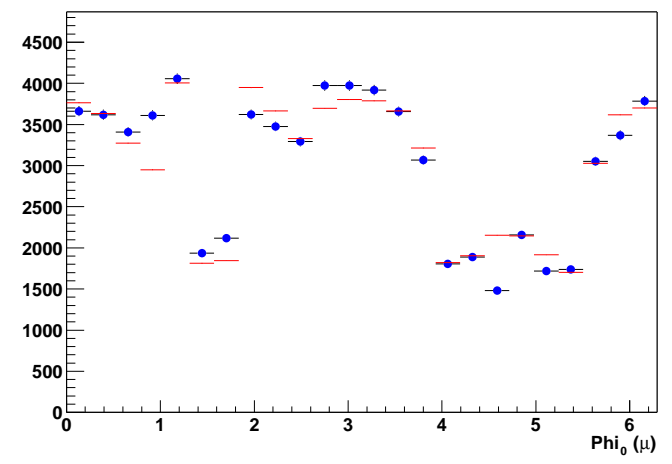

Figure 5.11: a) The comparison of data and $\operatorname{simulation} \cot \theta$ and b) $\phi_{0}$ distributions. 
as well as the energy from multiple interactions in the same bunch crossing. The second contribution is usually referred to as the "underlying event". The response of the calorimeter to the recoil is described by a response function, $R$, in the detector simulation, which scales the true recoil magnitude to the measured magnitude. The response function is parametrized as:

$$
R=\frac{u_{\text {rec }}}{u_{\text {true }}}=a+b \cdot u_{\text {true }}-c \cdot u_{\text {true }}^{2}
$$

where $u_{r e c}$ is the reconstructed recoil to be modeled, $u_{\text {true }}$ is the generated, true, transverse momentum of the Boson and $a, b$ and $c$ are positive constants obtained from comparisons to the data. The response function describes an inverted parabola. At the point when $u_{\text {true }}>\frac{b}{2 c}, R$ becomes non-monotonic and the derivative changes sign. Since the detector response does not decrease with increasing energy in the calorimeter, $R$, is allowed to increase to its maximum value at $u_{\text {true }}=\frac{b}{2 c}$ and remains constant thereafter.

The recoil resolution is parametrized with the assumption that there are two components. A sampling term that represents the calorimeter resolution to jets and a resolution that represents underlying event. In analogy to the resolution for jets measured in the calorimeter the sampling resolution is parametrized as

$$
\sigma^{s}=s_{\text {had }} \cdot \sqrt{u_{\text {true }}}
$$

where $s_{\text {had }}$ is the sampling term, that is determined from the data. The sampling resolution is added to the reconstructed recoil as a Gaussian distribution with a mean of zero and a width of $\sigma^{s}$.

The (soft) underlying event contribution to the recoil resolution is modeled using a minimum bias sample. Minimum bias events are triggered on a minimal detector requirement, essentially hits in the Cherenkov beam counters located near the beam axis. Minimum bias events are therefore mostly inelastic proton-antiproton collisions with small momentum transfer. In the detector simulation, the underlying event contribution 
is represented by a random vector $e^{u e}$, given by

$$
e_{i}^{u e}=A_{i}+B_{i} \cdot \Sigma E_{T} \oplus \sigma_{i}\left(\Sigma E_{T}\right)
$$

where the linear parameters $A_{i}$ and $B_{i}$ are obtained from the minimum bias data (with $i$ being either $x$ or $y$ ) and $\Sigma E_{T}$ is the sum over all calorimeter towers,

$$
\Sigma E_{T}=\Sigma_{\text {towers }} E \sin \theta=\Sigma_{\text {towers }} E_{T}
$$

The underlying event resolution is independently smeared for $\sigma_{x}^{u e}$ and $\sigma_{y}^{u e}$ according to a Gaussian distribution with mean zero and width $\sigma_{i}\left(\Sigma E_{T}\right)$. The $\Sigma E_{T}$ distribution is parametrized as the following function $P\left(\Sigma E_{T}\right)$ in the simulation.

$$
P\left(\Sigma E_{T}\right)=\left(\Sigma E_{T}\right)^{m} \cdot e^{-\frac{\Sigma E_{T}}{n}}
$$

where $m$ and $n$ are fit parameters obtained from the minimum bias data. The minimum bias data was chosen to have the same distribution of instantaneous luminosity as the $W$ and $Z$ data. Figure 9.4 shows the instantaneous luminosity overlayed for minimum bias, $W$ and $Z$ events.

Since the mean number of interactions in minimum bias events is different from $W$ and $Z$ events, the parametrized $\Sigma E_{T}$ is corrected for this difference. In this model we

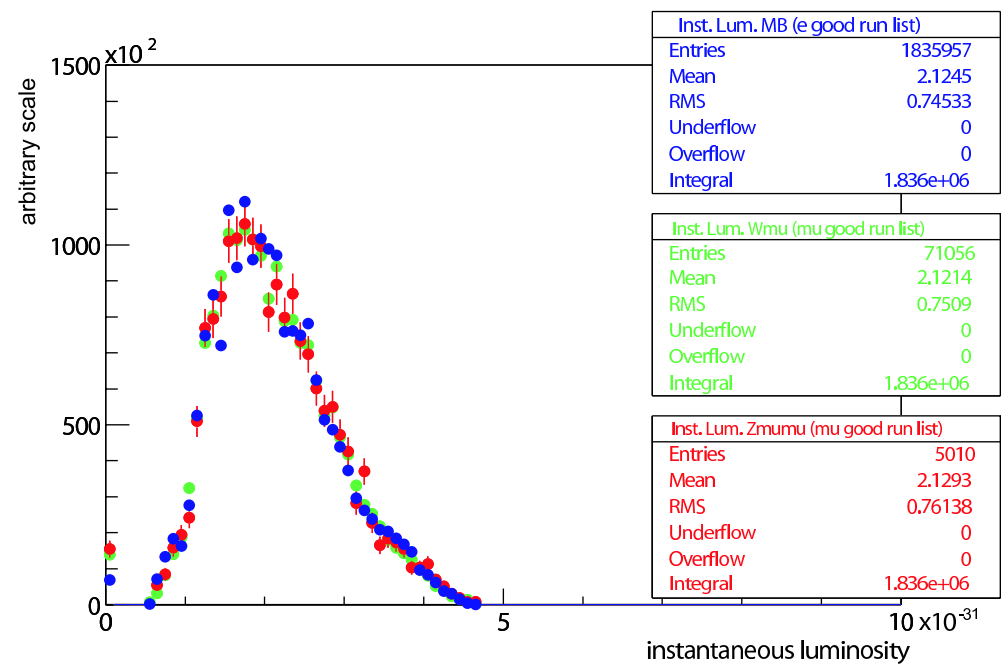

Figure 5.12: Instantaneous luminosity for minimum bias, $W$ and $Z$ events. 
separate the hard interaction, that produces the $W$ Boson from additional minimum bias events that can happen in the same bunch crossing (multiple interactions). This is achieved by reducing the $\Sigma E_{T}$ distribution from minimum bias events to that of a single interaction. Then we sample once from the single interaction distribution, and again from the original $\Sigma E_{T}$ distribution from the minimum bias data for the fraction of events corresponding to one or more additional interactions.

\subsubsection{Underlying Event}

When calculating the recoil vector by summing over calorimeter towers, the energy deposited by the muon has to be excluded from the sum. The $W \rightarrow \mu \nu$ data is used to measure the energy deposition of the muon in the calorimeter. Figure 5.13 shows the mean $E_{T}$ in the electromagnetic towers and hadronic calorimeter towers in the vicinity of the extrapolated muon track in a grid of $\eta$ and $\phi$, averaged over all muon candidates. The grid is adjusted to be centered on each muon candidate. The distributions in both, the electromagnetic and hadronic calorimeter show that most of the muon energy is de-
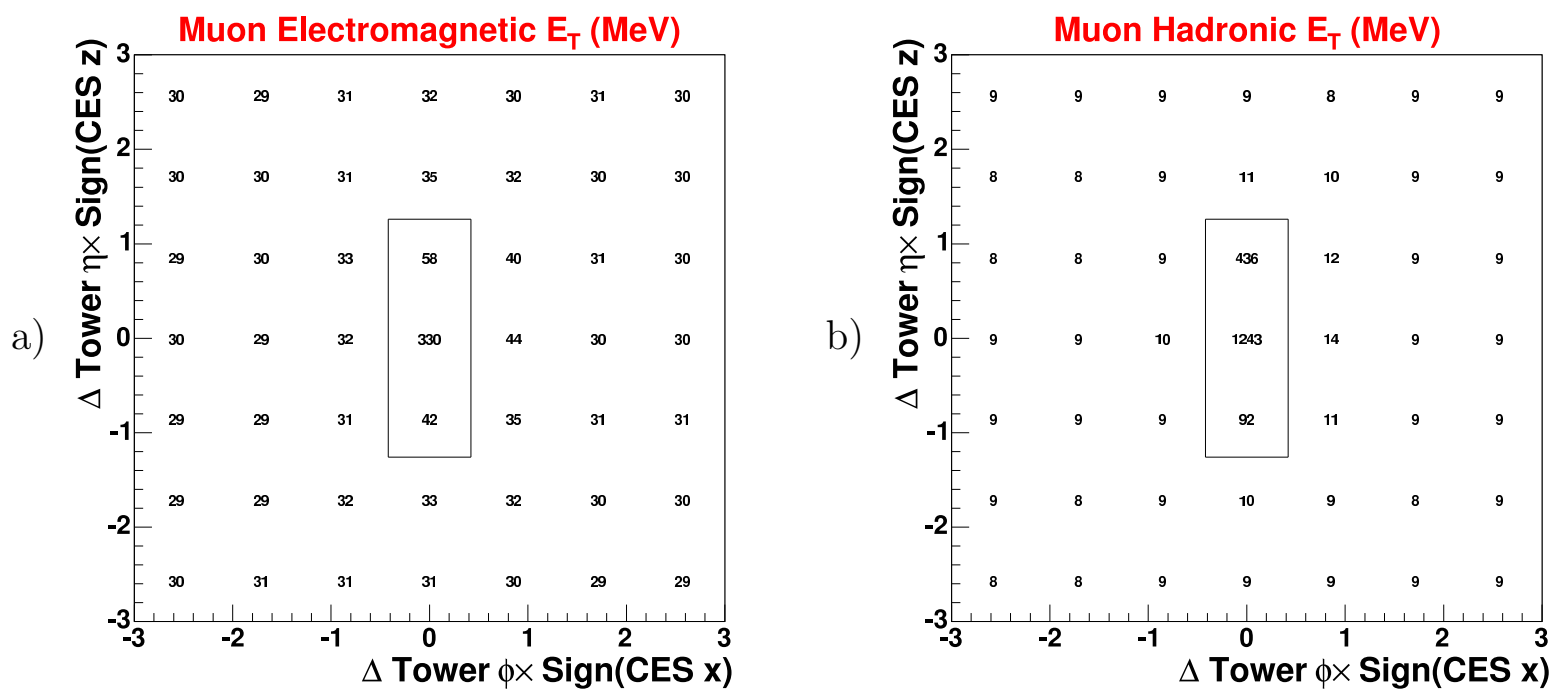

Figure 5.13: a) The mean $E_{T}$ in the electromagnetic calorimeter towers and b) hadronic calorimeter towers in the vicinity of the muon track. 
posited in three adjacent $\eta$ towers. The towers surrounding the three indicated muon towers contain mostly energy from the underlying event. Removing the three muon towers from the recoil calculation therefore also removes the underlying event energy in those three towers. To match the data, the same is done for the recoil calculation in the simulation. The underlying event energy is measured using equivalent towers separated from the muon candidate towers by two or more towers in $\phi$. The variation of the measured energy with $\phi$ is $10 \mathrm{MeV}$ and is taken as the systematic uncertainty for extracting the amount of underlying event energy from the data.

The geometric size of the calorimeter towers varies with $\eta$. As a result, the underlying event energy measured in each tower also varies with $\eta$, as can be seen in Figure 5.15. This dependence is parametrized and included in the simulation. The underlying event energy flow into the muon towers also depends on the overlap between the direction of the recoil and the muon. A measure for the recoil overlap with the muon is the component of the total recoil in the direction of the muon, called $u_{\|}$. Figure 5.14 illustrates the

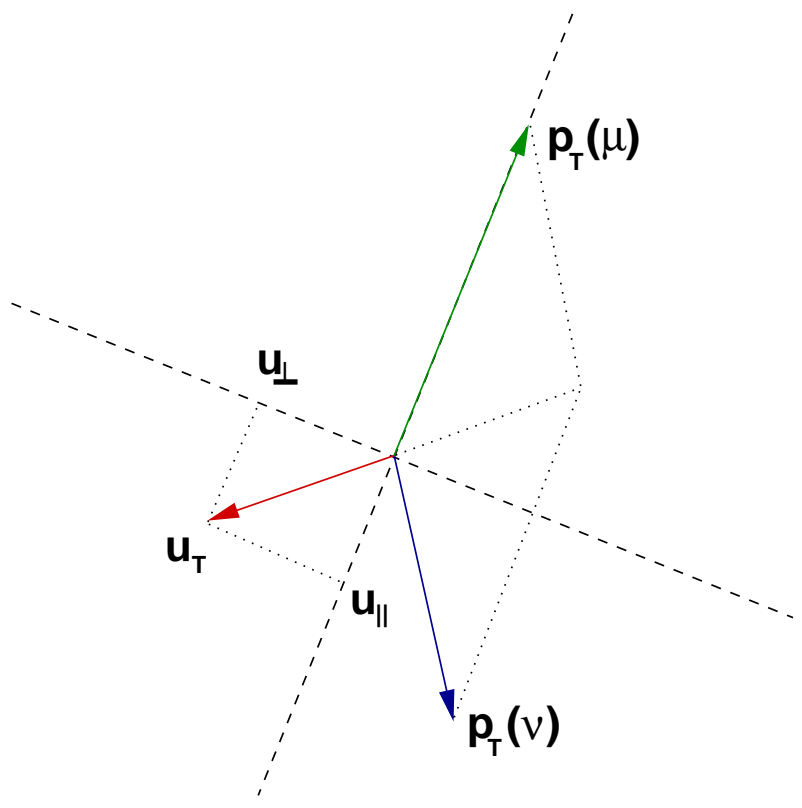

Figure 5.14: Illustration of momentum vectors in the transverse plane and components of the $u$ vector parallel and perpendicular to the muon. 
momentum vectors in the transverse plane and components of the $\mathbf{u}$ vector parallel and perpendicular to the muon. To first order, the transverse mass of the $W$ Boson can be written as $M_{T}=2 p_{T}(\mu)+u_{\|}$. Any bias in $u_{\|}$therefore translates directly into a bias of the transverse mass. The underlying event energy is also studied as function of $u_{\|}$. Figure 5.16 shows the variation of $E_{T}^{u e}$ in the removed muon towers with $u_{\|}$. One can clearly see an increase in the underlying event energy as $u_{\|}$increases. This is due to the additional recoil particles that enter the muon tower. This dependence is included as a parametrization in the fast detector simulation.

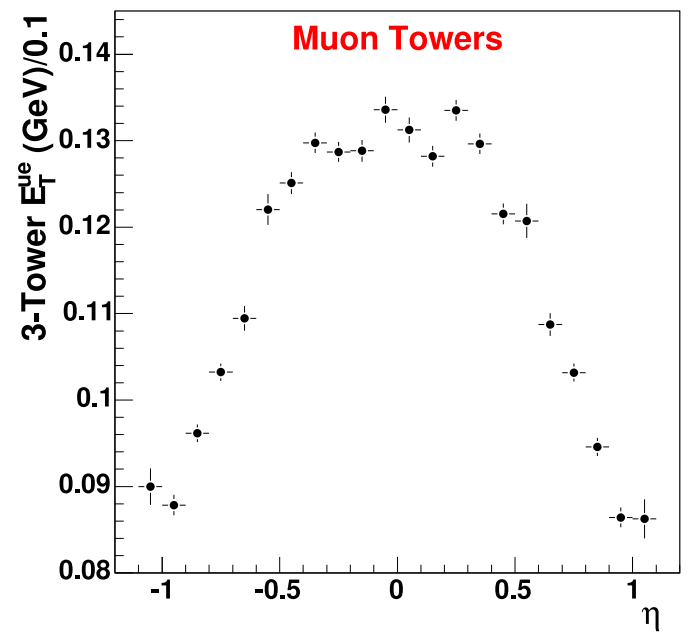

Figure 5.15: Variation of $E_{T}^{u e}$ in the removed muon towers with $\eta$.

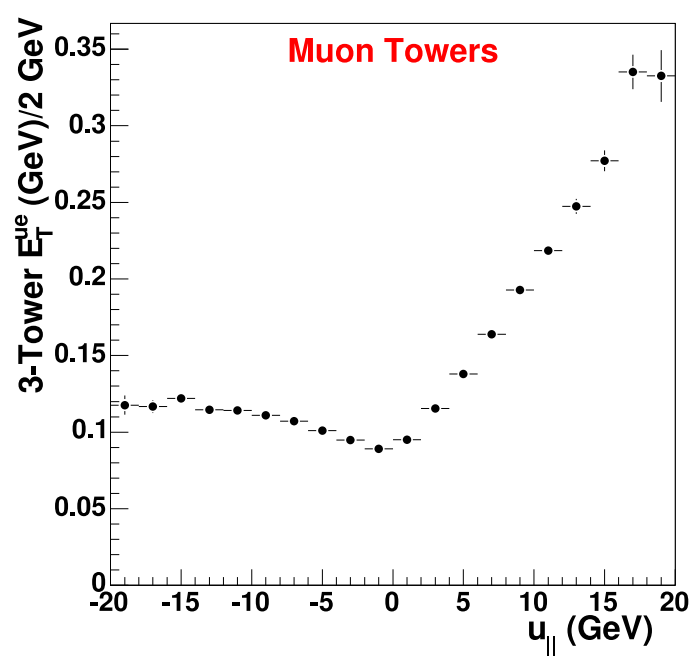

Figure 5.16: Variation of $E_{T}^{u e}$ in the removed muon towers with $u_{\|}$.

\subsubsection{Efficiency Modeling}

The muon identification is also influenced by the recoil. We invesigate the identification efficiency as a function of $u_{\|}$. The muon identification efficiency is measured using $Z \rightarrow$ $\mu \mu$ candidate events. One of the two muon candidates is selected according to the full muon candidate selection, as described in chapter 4 . For the second muon candidate in the event, only a transverse momentum $>25 \mathrm{GeV} / \mathrm{c}$, a $z_{0}<60 \mathrm{~cm}$, a muon chamber stub, 
and a matching track traversing all superlayers is required. To reduce backgrounds, only events from a narrow mass region $\left(86 \mathrm{GeV} / \mathrm{c}^{2}<m_{\mu \mu}<96 \mathrm{GeV} / \mathrm{c}^{2}\right)$ are used. Figure 5.17 shows the identification efficiency as a function of $u_{\|}$. As one would expect, the identification efficiency decreases with increasing $u_{\|}$. The distribution is fit to the function: $\epsilon=a\left[1+b\left(u_{\|}-6+\left|u_{\|}-6\right|\right)\right]$. The resulting parameter $b$ from the fit is also shown in Figure 5.17.

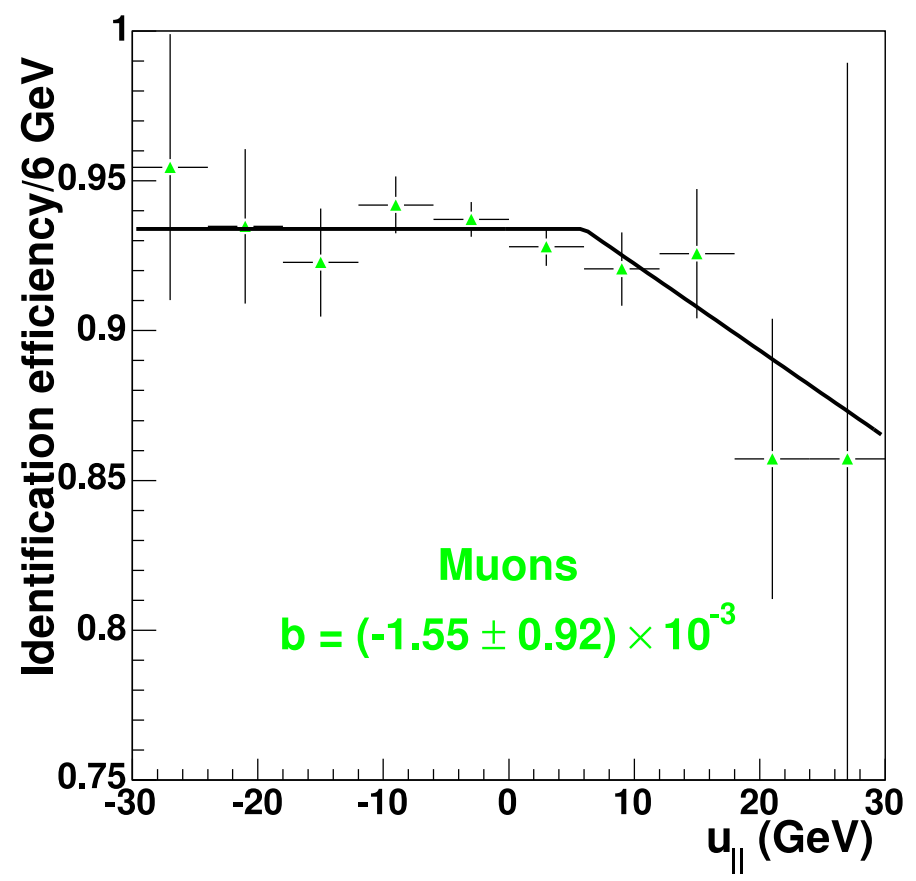

Figure 5.17: Variation of muon identification efficiency with $u_{\|}$. 


\section{Chapter 6}

\section{Momentum Calibration}

The muon momentum is the most important quantity for the $W$ mass measurement in the muon decay channel since the bulk of the transverse mass of the $W$ Boson is computed from the transverse momentum of the muon track. The muon momentum is measured in the Central Outer Tracker (COT). We use the high statistics $J / \Psi$ and $\Upsilon$ quarkonium resonances to set the momentum scale. The momentum scale is obtained from the deviation of the measured particle mass from the more precisely known world average values $M_{J / \Psi}=3096.87 \pm 0.04 \mathrm{MeV}[43]$ and $M_{\Upsilon(1 S)}=9460.30 \pm 0.26 \mathrm{MeV}$ [44]. The calibration of the tracker involves several steps which will be explained in more detail in this chapter.

\subsection{Tracker Alignment}

The internal alignment of the COT is performed using a large sample of cosmic rays. The cosmic ray events are identified as events with only one or two tracks, at least one of which has a stub in a muon chamber, and is tagged as a cosmic ray by the cosmic tagger [45]. Once identified as a cosmic ray both tracks in the upper and lower half of the COT can be reconstructed by a specialized cosmic ray track fit which does not require that the track originated from the beam collision. A single helix is then fit to both tracks, and 
the residuals between the track and the hits are used to measure the misalignment of each cell. Collider data are used to ensure that the global $(x, y)$ coordinates of the COT are consistent with the COT beamline. In addition to the alignment in $r-\phi$, a binned $z$-dependent alignment is performed to account for wire sag and electrostatic deflection of the wires. Residual misalignments are checked with electrons from $W \rightarrow e \nu$ decays. The ratio of electron energy $E$, as measured in the calorimeter, and electron momentum $p$, as measured in the COT, $(E / p)$ is computed separately for electrons and positrons. A residual misalignment manifests itself in a non-zero difference between electrons and positrons, since the calorimeter measurement is unaffected by the misalignment. Based on the $E / p$ difference, a curvature correction is determined to make the difference vanish. The resulting correction is parametrized as a quadratic function in $\cot \theta$ :

$$
\Delta\left(q / p_{T}\right)=c_{0}+c_{1} \cot \theta+c_{2} \cot ^{2} \theta,
$$

where $q$ is the charge of the track. The parameters and their uncertainties are shown in Table 6.1. The coefficients $c_{0}, c_{1}$ and $c_{2}$ are varied by their uncertainties in the simulation.

\begin{tabular}{lc}
\hline Parameter & Fitted value \\
\hline$c_{0}$ & $-(4.5 \pm 1.5) \cdot 10^{-5}$ \\
$c_{1}$ & $-(7.0 \pm 1.5) \cdot 10^{-5}$ \\
$c_{2}$ & $(2.45 \pm 0.25) \cdot 10^{-4}$ \\
\hline
\end{tabular}

Table 6.1: The parameters of the coefficients to the curvature correction function.

We then fit the resulting simulated $W$ events with unchanged templates. The systematic uncertainty on the $W$ Boson mass resulting from these variations is $20 \mathrm{MeV} / \mathrm{c}^{2}$.

\subsection{Momentum and $z$ Scales using $J / \Psi \rightarrow \mu \mu$ Events}

The momentum scale is extracted from a binned maximum likelihood fit to an invariant mass template. The templates are generated as a function of the momentum scale devi- 
ation from unity $\Delta p=p-1$. Figure 6.1 shows a template with invariant mass spectra in the range of $\Delta p= \pm 0.004$ separated in 500 bins.

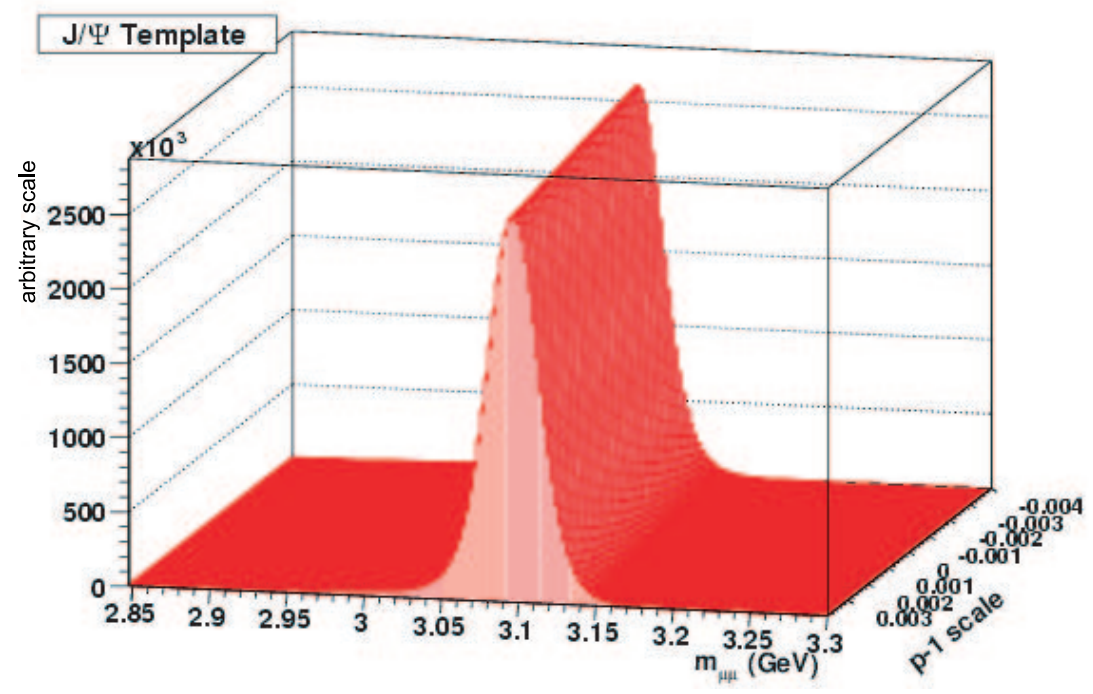

Figure 6.1: Template for $J / \Psi$ momentum scale fit.

The fit result is obtained by computing the likelihood between the $J / \Psi \rightarrow \mu \mu$ invariant mass distribution reconstructed for the data and each simulated template. Both the data and simulation are binned. The likelihood function is derived from a Poisson distribution

$$
P_{\lambda}(n)=\frac{\lambda^{n} e^{-\lambda}}{n !}
$$

which gives the probability of observing $n$ given a prediction of $\lambda$. Stirling's formula $n ! \approx n \cdot \ln (n)-n$ is used to express the factorial in a numerical way. Taking the logarithm, we get the following equation which is used in the fitting procedure:

$$
\ln (P)=\Sigma_{i}\left(-N_{M C}^{i}+N_{\text {data }}^{i} \cdot \ln \left(N_{M C}^{i}\right)-N_{\text {data }}^{i} \ln \left(N_{\text {data }}^{i}+1\right)-N_{\text {data }}^{i}\right),
$$

where $N_{d a t a}^{i}$ and $N_{M C}^{i}$ represent the bin contents in the data and simulation respectively. The likelihood function is evaluated for all simulated invariant mass histograms in the template and the minimum represents the measured $\Delta p$. The uncertainty is estimated by taking the average of the scales found for a \pm 0.5 increase in $\ln (P)$. 
The large statistics of the $J / \Psi$ sample $(\sim 400000)$ allows us to study the momentum scale as a function of other variables which can be used to calibrate the COT. One such variable is the difference in $\cot \theta$ between the positively and negatively charged muon track. Figure 6.2 shows the variation of $\Delta \mathrm{p}$ with $\Delta \cot \theta$, fitted to a parabola. A linear

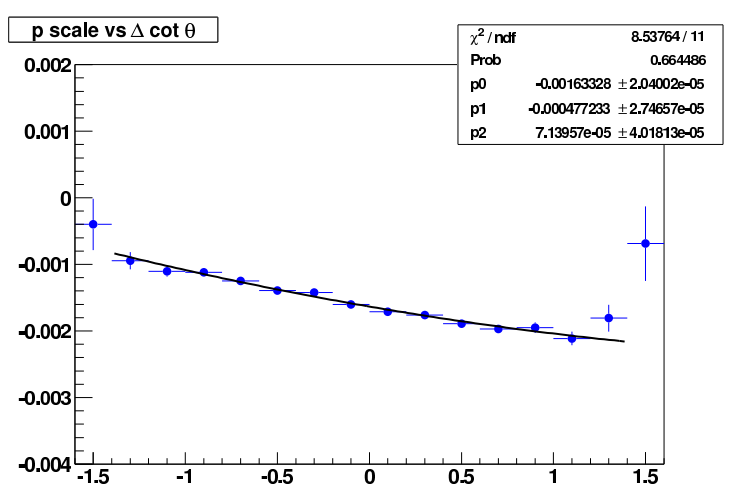

Figure 6.2: Fitted $\Delta \mathrm{p}$ as a function of $\Delta \cot \theta$, before corrections, fitted to a parabola.

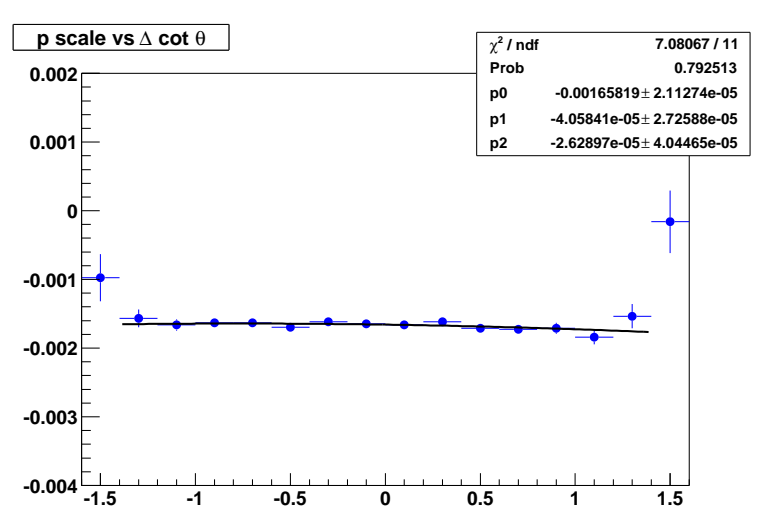

Figure 6.3: Fitted $\Delta \mathrm{p}$ as a function of $\Delta \cot \theta$, after corrections, fitted to a parabola.

variation can be explained by a relative rotation of the east and west endplates of the COT. A quadratic variation can result from a small deviation of the stereo angles from their nominal value. Making the following corrections to $\cot \theta$ and the curvature $c$ in the data

$$
\begin{aligned}
& \cot \theta \rightarrow(0.9998 \pm 0.0001) \cdot \cot \theta \\
& c \rightarrow c-(6.0 \pm 0.3) \cdot 10^{-7} \cot \theta
\end{aligned}
$$

eliminates both the linear and the quadratic dependences of $\Delta \mathrm{p}$ on $\Delta \cot \theta$. This is demonstrated in Figure 6.3.

An incorrect accounting for material in the detector description causes the reconstructed mass of the $J / \Psi$ resonance to depend on the average transverse momentum of the decay muons. The correction for this effect involves a two-step procedure. In the first step the dependence of $\Delta \mathrm{p}$ on the transverse momentum is eliminated by adding material 
to the tracking volume description (the overall material can be scaled in silimap). This checks the simulation of the ionization energy loss. If it is correctly simulated, there should be no dependence. Figure 6.4 shows the variation of $\Delta p$ with the average transverse momentum of the two decay muons from the $J / \Psi$ after scaling the overall material in the simulation to $85 \%$ of itself. The scaling of the material description causes for example a track with transverse momentum of $5 \mathrm{GeV} / \mathrm{c}$ to loose about $9 \mathrm{MeV}$ through energy loss. This amount of energy loss is confirmed by a cosmic ray study [46]. In this study, the energy loss is measured directly by comparing the transverse momentum of the incoming and outgoing tracks from cosmic rays. The energy loss from this data measurement can be compared to the energy loss experienced by a particle in the simulation, which uses silimap. The energy loss agrees within uncertainties.

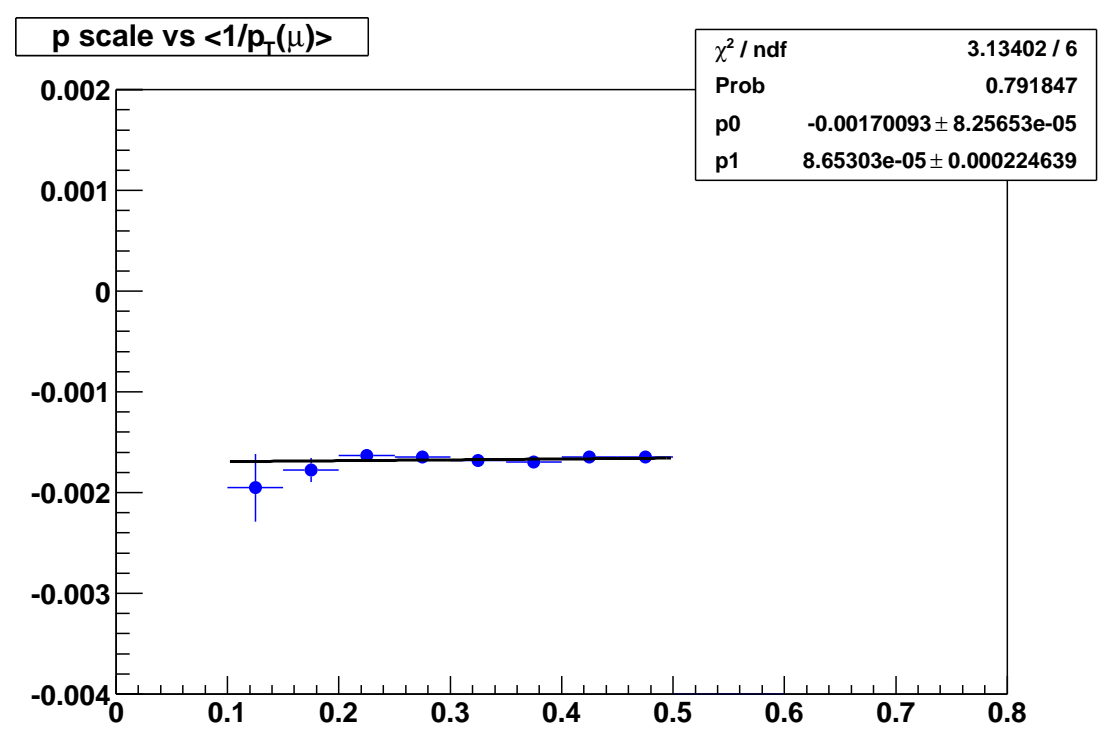

Figure 6.4: Fitted $\Delta \mathrm{p}$ as a function of $\left\langle 1 / p_{T}^{\mu}\right\rangle$, fitted to a straight line.

In the second step, the magnetic field is calibrated by requiring that the reconstructed $J / \Psi$ mass be equal to the world average. The average $p_{T}$ for $W$ and $Z$ muons is about $36 \mathrm{GeV} / \mathrm{c}$ and $41 \mathrm{GeV} / \mathrm{c}$, which corresponds to $0.027 \mathrm{c} / \mathrm{GeV}$ and $0.024 \mathrm{c} / \mathrm{GeV}$ in $1 / p_{T}$ space respectively. The intercept of the fit at $\left\langle 1 / p_{T}^{\mu}\right\rangle=0$ in Figure 6.4 is $\Delta p / p=$ $(-1.70 \pm 0.08$ (stat) $) \cdot 10^{-3}$, which is the measured momentum scale from the $J / \Psi$ sample. 


\subsection{COT Momentum Scale using $\Upsilon \rightarrow \mu \mu$ Events}

With an invariant mass of the order $10 \mathrm{GeV} / c^{2}$, the $\Upsilon(1 S)$ resonance supplies a relatively high statistics intermediate reference point to study the momentum scale between the over three times lighter $J / \Psi$ resonance and the over nine times heavier $Z$ Boson. Figure 6.5 shows the dimuon mass spectrum from the data between 8.8 to $11.2 \mathrm{GeV} / \mathrm{c}^{2}$ fit with three Gaussian distributions and a linear background. The three upsilon resonances

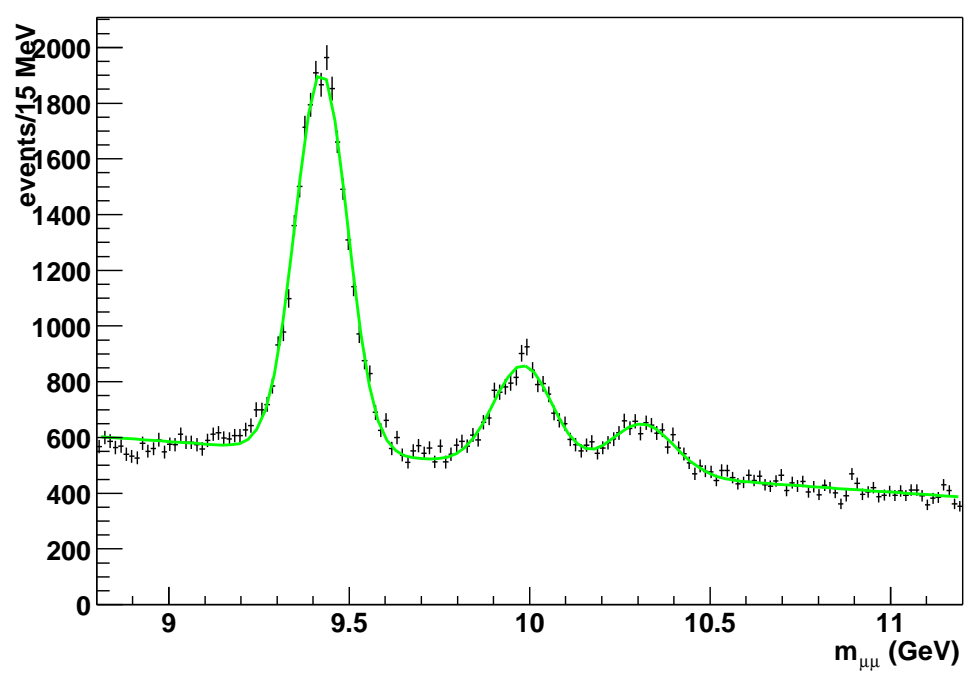

Figure 6.5: Dimuon mass spectrum with a fit of three Gaussian distributions and a linear background

$\Upsilon(1 \mathrm{~S}), \Upsilon(2 \mathrm{~S})$ and $\Upsilon(3 \mathrm{~S})$ are clearly visible. The background underneath the resonances is well modeled with a linear parametrization which is incorporated in the fast detector simulation. Due to the much larger statistics of the $\Upsilon(1 S)$, it is the only one out of the three which we use to extract a momentum scale. To generate the events with a more realistic $p_{T}$ distribution, the $p_{T}$ distribution coming from PYTHIA is fine tuned by hand (see chapter 5) to better match the data. Figure 6.6 and 6.7 show the comparison of the $\Upsilon(1 S)$ data with the simulation of the $p_{T}$ and $p_{z}$ distributions respectively. The data in the figures have backgrounds subtracted using sidebands. Since the events to 

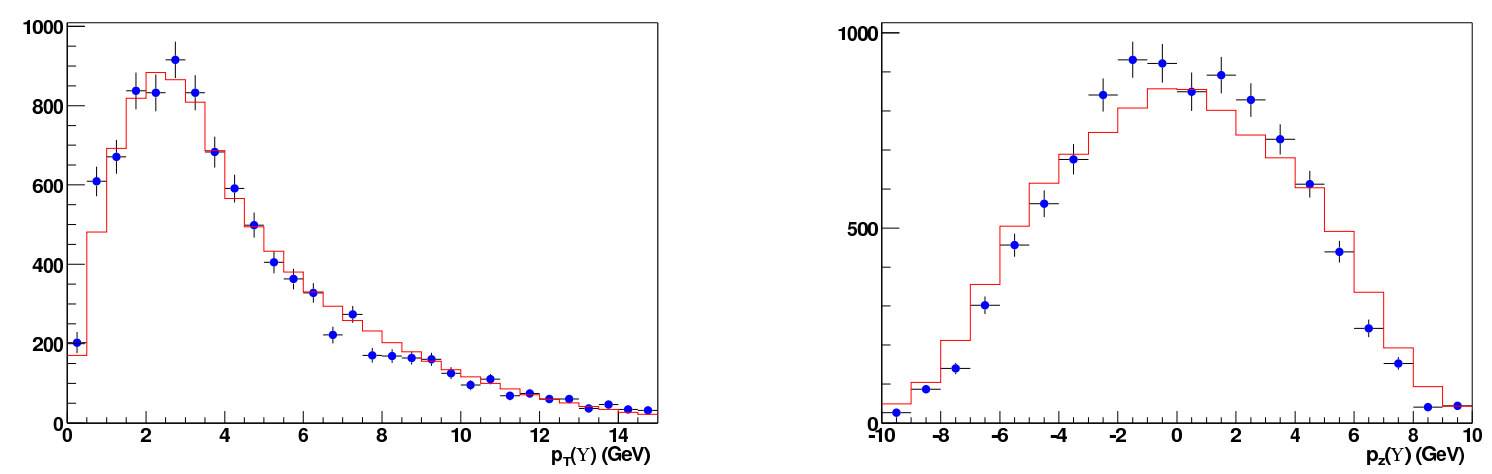

Figure 6.6: Comparison of data with Figure 6.7: Comparison of data with Monte Carlo of the $\Upsilon(1 \mathrm{~S}) p_{T}$ distribution. $\quad$ Monte Carlo of the $\Upsilon(1 \mathrm{~S}) p_{z}$ distribution.

the left and right of the resonance (sidebands) are expected to have similar kinematics to the background events underneath the resonance, we use them in the background subtraction. The signal region is chosen to be from 9.30 to $9.56 \mathrm{GeV} / \mathrm{c}^{2}$ and half the sidebands are taken from the lower sideband region $\left(9.17\right.$ to $\left.9.30 \mathrm{GeV} / \mathrm{c}^{2}\right)$ and upper sideband region (9.56 to $9.69 \mathrm{GeV} / \mathrm{c}^{2}$ ). Figures 6.8 and 6.9 show comparisons of the data with the simulation of the $\Upsilon(1 \mathrm{~S})$ decay muon $p_{T}$ and $p_{z}$ distributions respectively. The templates for the $\Upsilon$ mesons are produced in exactly the same way as for the $J / \Psi$ meson analysis. For the upsilon template generation we also scale the amount of material that

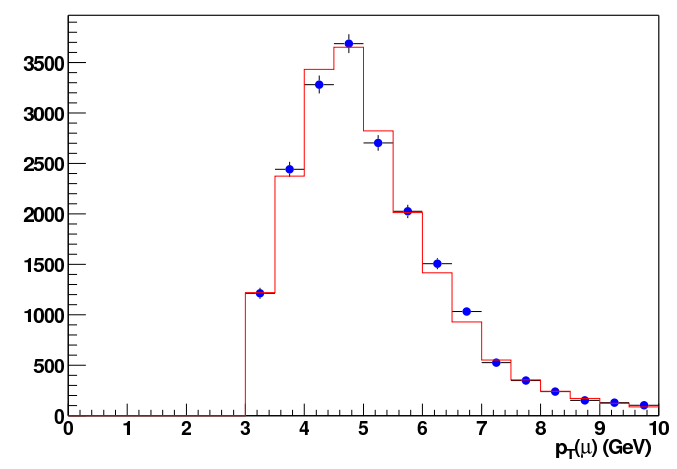

Figure 6.8: Comparison of the muon candidate $p_{T}$ distribution.

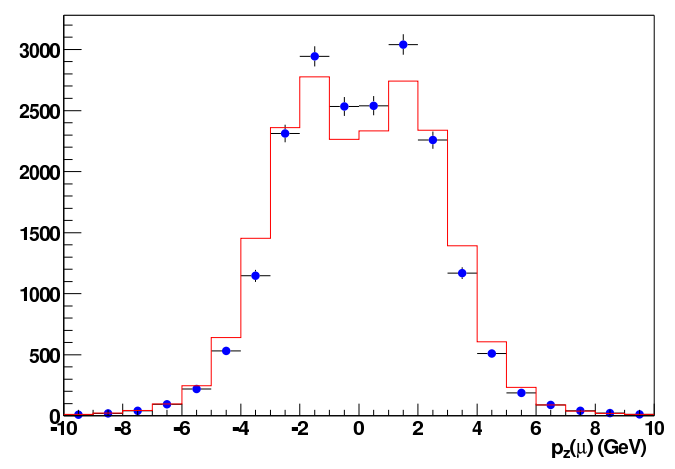

Figure 6.9: Comparison of the muon candidate $p_{z}$ distribution. 


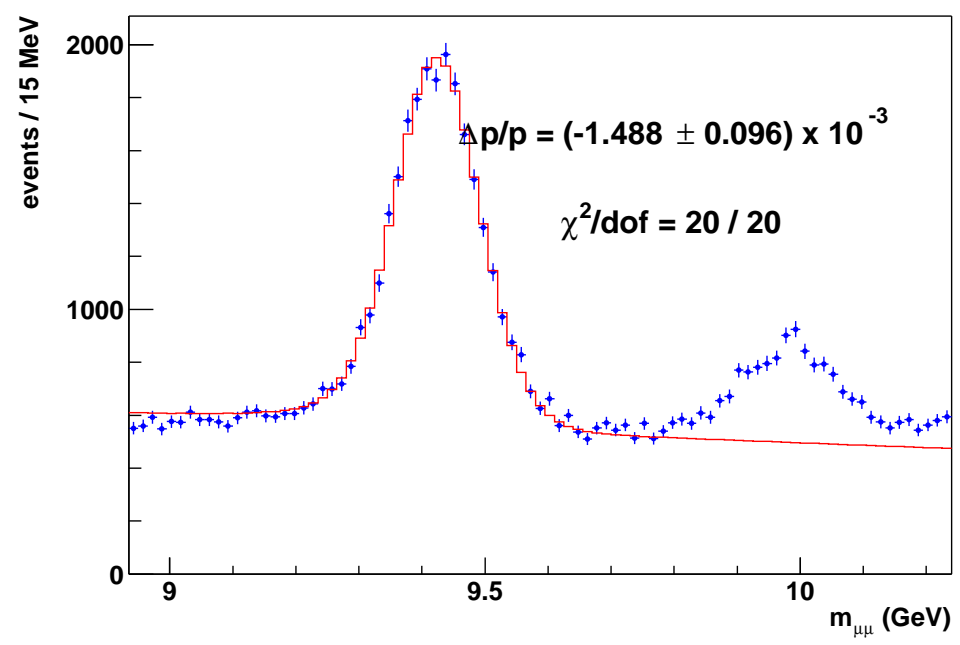

Figure 6.10: The invariant mass distribution with the best fit simulated template and the extracted momentum scale using non beam constrained quantities.

causes energy loss through ionization to $85 \%$ of itself, as derived from the $J / \Psi$ analysis.

Since upsilon mesons are only produced promptly, we can extract the momentum scale for two invariant mass distributions, one which uses beam constrained tracks and the other using COT only tracks, thus testing for a possible systematic bias. Figure 6.10 shows the fit using the non beam constrained quantities and Figure 6.11 shows the

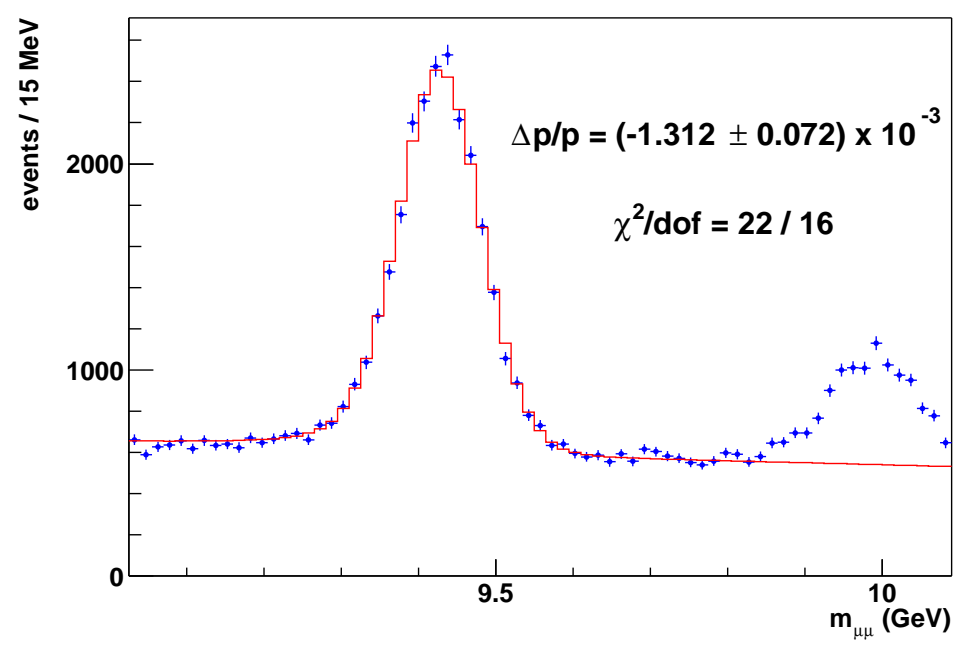

Figure 6.11: The invariant mass distribution with the best fit simulated template and the extracted momentum scale using beam constrained quantities. 
fit to the invariant mass distribution using beam constrained quantities. The former is wider due to the worse resolution. The quoted uncertainties are statistical only. We also investigate possible systematic uncertainties. A list of these is presented in Table 6.2. The

\begin{tabular}{ccc}
\hline Source of Uncertainty & Uncertainty in ppt & Comment \\
\hline Statistical & 0.10 & from fit \\
\hline Beam Constrained Fit & 0.18 & difference in bc and non bc fit \\
QED Radiative Effects & 0.10 & varying fit range \\
Muon Energy Loss & 0.05 & shift in mass due to $\pm \sigma_{E L o s s}^{J / \Psi}$ \\
p cut variations & 0.02 & shift mass due to $\mathrm{p}_{T}$ cut $\pm 100 \mathrm{MeV} / \mathrm{c}$ \\
Background Shape & 0.01 & shift in mass for different slopes \\
\hline Combined Systematics & 0.21 & \\
\hline
\end{tabular}

Table 6.2: Systematic uncertainties in parts per thousand (ppt) for $\Upsilon(1 S)$ fit

difference in the fitted scale using beam constrained and non beam constrained tracks is taken as a systematic uncertainty on the track fitting simulation. To estimate the systematic uncertainty arising from mismodeling QED radiation, we vary the fit window in the low mass region, where QED effects alter the lineshape the most, while keeping the high end of the fit range fixed. For the uncertainty in the muon energy loss, we vary the material scale by its statistical uncertainty as obtained from the $J / \Psi$ measurement in Figure 6.4. To obtain an uncertainty on mismodeling event kinematics, we vary the muon $p_{T}$ cut by $100 \mathrm{MeV} / \mathrm{c}$ in the data and the simulation and note the change in scale. For the background uncertainty, we vary the background slope in the fit by its uncertainty. Adding all contributions in quadrature, a total systematic uncertainty of 0.21 parts per thousand is found.

Table 6.3 summarizes all the measured momentum scales. To combine the momentum 


\begin{tabular}{ccc}
\hline & $J / \Psi$ & $\Upsilon(1 S)$ \\
\hline Momentum Scale $(\Delta p / p)$ & $(-1.70 \pm 0.08) \cdot 10^{-3}$ & $(-1.49 \pm 0.10) \cdot 10^{-3}$ \\
Beam constrained & N $/ \mathrm{A}$ & $(-1.31 \pm 0.07) \cdot 10^{-3}$ \\
\hline
\end{tabular}

Table 6.3: Measured momentum scales

scales we take the average of the two upsilon results for beam constrained and non beam constrained tracks and average it with the momentum scale found from the $J / \Psi \rightarrow \mu \mu$ analysis. The combined statistical uncertainty from the $J / \Psi \rightarrow \mu \mu$ and $\Upsilon(1 S) \rightarrow \mu \mu$ is added to the systematic uncertainty. This results in an overall momentum scale value of $\Delta p / p=(-1.55 \pm 0.22) \cdot 10^{-3}$. On the $W$ mass this introduces a $18 \mathrm{MeV} / c^{2}$ systematic uncertainty. Combined with the $20 \mathrm{MeV} / \mathrm{c}^{2}$ uncertainty from the misalignment, we obtain a total systematic uncertainty, associated with the momentum determination, of $27 \mathrm{MeV} / c^{2}$.

\subsection{Momentum Scale Cross-Check and Momentum Resolution using $Z \rightarrow \mu \mu$ Events}

We apply the COT momentum scale correction factor of 1.00155 to each of the beam constrained muon tracks, to reconstruct the dimuon kinematics in $Z \rightarrow \mu \mu$ events. We perform a maximum likelihood fit for the measured $Z$ pole mass, where the fitting window ranges from 84 to $98 \mathrm{GeV} / c^{2}$. The $Z \rightarrow \mu \mu$ mass fit is shown in Fig. 6.12, with its statistical error. The systematic uncertainty due to tracker momentum scale is found to be $28 \mathrm{MeV} / \mathrm{c}^{2}$. Hence the result from the $Z \rightarrow \mu \mu$ mass fit is:

$$
M_{Z}=91163 \pm 47_{\text {stat }} \pm 28_{\text {tracker }}=(91163 \pm 55) \mathrm{MeV} / \mathrm{c}^{2}
$$

The result agrees well with the world-average $Z$ mass, $M_{Z}=(91188 \pm 2) \mathrm{MeV} / \mathrm{c}^{2}[47]$. 


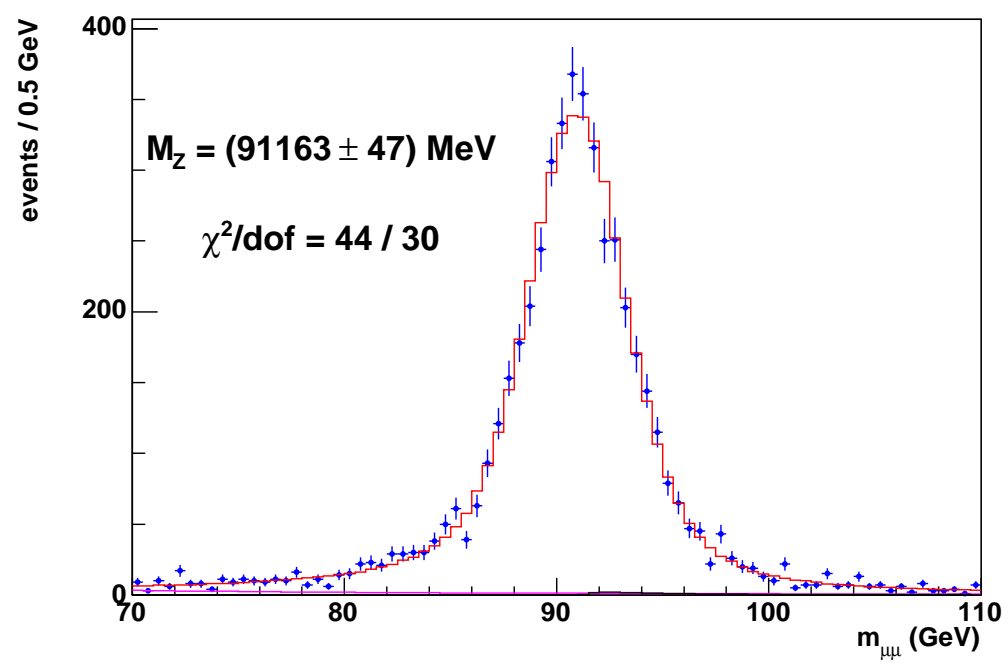

Figure 6.12: The $Z \rightarrow \mu \mu$ invariant mass fit.

The tracker resolution is tuned using the width of the $Z$ mass peak. The resolution is parametrized in the simulation by a Gaussian COT hit resolution, $\sigma_{h}$. We vary this parameter so that the $\chi^{2}$ of the $Z$ mass distribution is minimized, and let the $\chi^{2}$ increase by one to obtain the error on $\sigma_{h}$. We obtain $\sigma_{h}=(200 \pm 15) \mu \mathrm{m}$. 


\section{Chapter 7}

\section{Backgrounds}

Backgrounds passing the event selection cuts have very different transverse mass distributions than the $W$ Boson signal. In order not to bias the mass fit, the various backgrounds have to be included, with the appropriate normalizations, in the simulated templates used to extract the $W$ Boson mass. For systematic uncertainties on the $W$ Boson mass, we consider the uncertainties on the normalization and fitted shape of the various backgrounds. The largest background in the $W \rightarrow \mu \nu$ sample comes from $Z \rightarrow \mu \mu$ events, where one of the muon tracks is undetected. This is followed by $W \rightarrow \tau \nu$ events, where the tau decays into a muon and a neutrino. Other backgrounds come from kaon decays in flight, from QCD jet events, where one jet contains a non-isolated muon, and from cosmic rays entering the CDF detector.

\subsection{Cosmic Rays}

High energy muons originating from cosmic rays can produce a muon candidate that can be confused with a muon candidate from $W$ decay. Most of these events are removed by a specially designed cosmic tagger [45]. The cosmic tagger utilizes the fact that the two tracks originating from a cosmic ray do not coincide with the fixed beam bunch crossing time. It employs a multi-parameter fit to the hits in the COT including the track 
production time $t_{0}$ as a free parameter. We estimate the remaining cosmic background by examining the $t_{0}$ distribution of the refitted tracks for all $W$ signal candidates with the hypothesis that they originated from the center of the detector. We compare this distribution with events which were identified as cosmic rays by the cosmic tagger and with $Z \rightarrow \mu \mu$ events. These distributions are shown in Figure 7.1 for the $W$ signal region and in Figure 7.2 for the impact parameter $\left(d_{0}\right)$ sideband region $(0.2 \mathrm{~cm}$ to $0.6 \mathrm{~cm}$ and $-0.2 \mathrm{~cm}$ to $-0.6 \mathrm{~cm})$. For the identified cosmic rays, one would expect a flat $t_{0}$ distribution.

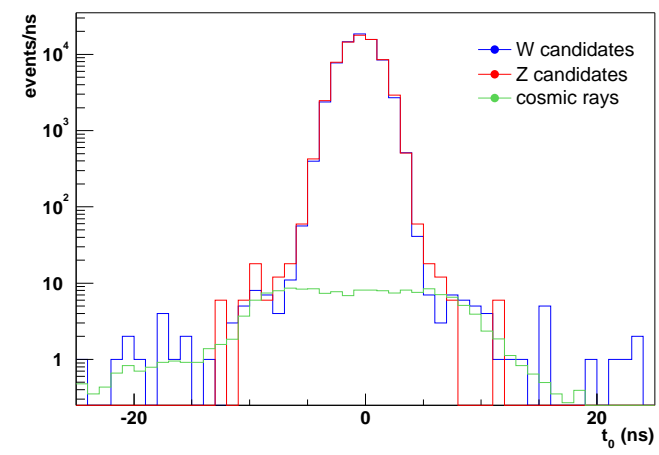

Figure 7.1: $t_{0}$ distribution for the outgoing track for all signal events.

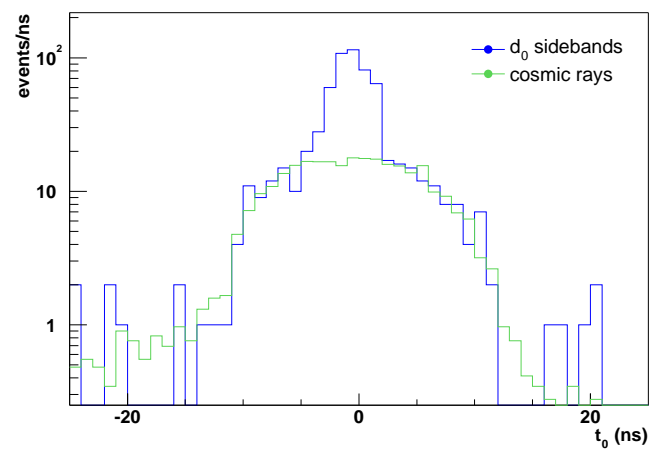

Figure 7.2: $t_{0}$ distribution for the outgoing track for $d_{0}$ sideband events.

Biases from the pattern recognition of the tracking algorithms, which favor tracks with small $t_{0}$, result in a plateau for $\left|t_{0}\right|$ values below $10 \mathrm{~ns}$. We estimate the size of the cosmic background in the $W$ candidate sample by normalizing the sideband regions of the $t_{0}$ distribution for the identified cosmic sample to the $W$ candidate sample. Table 7.1 shows the estimates applying different normalization regions for the $W$ signal region and for the $d_{0}$ sidebands of the signal region. It also shows the same plateau characteristic for the cosmic events as in the $W$ signal region. From the spread of the background size estimates in the two regions we obtain a cosmic background estimate of $(0.3 \pm 0.1) \%$.

We obtain the kinematic background shapes from identified cosmic rays. All $W$ Boson selection cuts are applied, with the exception of the cosmic tag, which is inverted. The $M_{T}$ distribution contains 8389 events and is shown in Figure 7.3. All background 


\begin{tabular}{lll}
\hline Normalization Region & Estimate in Signal Region & Estimate in $\mathrm{d}_{0}$ Sidebands \\
\hline $7-15 \mathrm{~ns}$ & $0.24 \pm 0.03$ & $0.28 \pm 0.03$ \\
$7-10 \mathrm{~ns}$ & $0.24 \pm 0.04$ & $0.28 \pm 0.03$ \\
$7-25 \mathrm{~ns}$ & $0.29 \pm 0.03$ & $0.29 \pm 0.03$ \\
$10-25 \mathrm{~ns}$ & $0.35 \pm 0.05$ & $0.30 \pm 0.03$ \\
\hline
\end{tabular}

Table 7.1: Cosmic background estimates for different normalization regions.

distributions are fit to multi-parameter functions, where we only allow the two parameters with the largest uncertainty to float. We then compute the correlation contour which is shown in the insert next to the distribution. The black curve shows the best fit to the data and the ten overlaid colored curves represent shapes obtained on the $3 \sigma$ uncertainty contour of the two parameter fit.

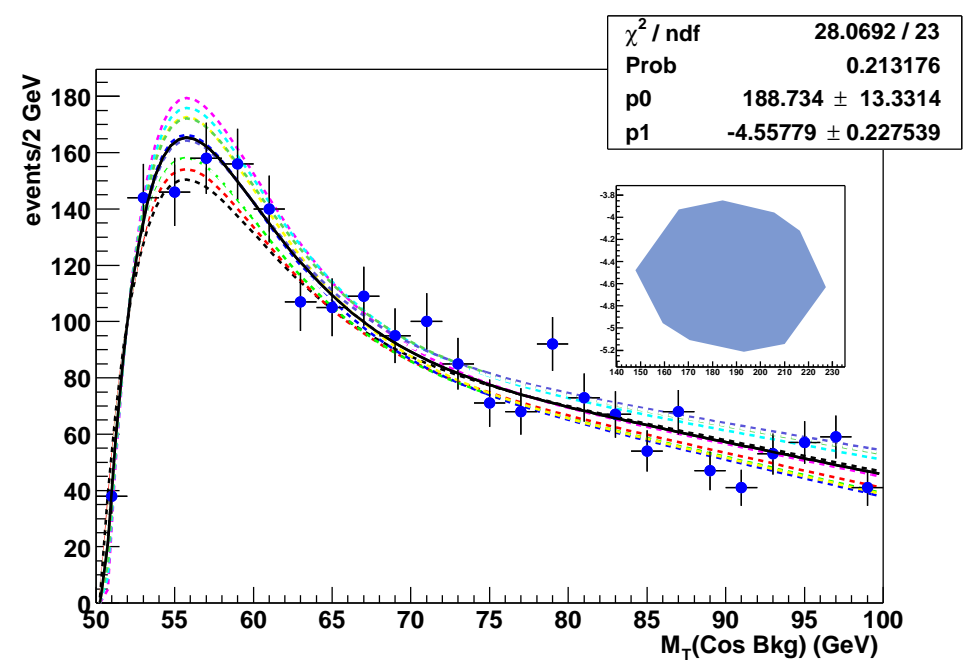

Figure 7.3: $M_{T}$ distribution for cosmic background. The best fit to the data and the $3 \sigma$ fitted shape uncertainty are overlaid. 


\subsection{Kaon Decays in Flight}

The $t_{0}$ distribution of the impact parameter sideband events in Figure 7.2 shows a peaked signal on top of the flat plateau originating from cosmic rays. This signal is thought to come from decays in flight, such as kaons decaying into muons. We estimate the contamination of this background in our signal region by evaluating the $\delta \phi(\mu, u)$ distribution, shown in Figure 8.7, which will be discussed in the next chapter. Only a small excess in the data can be observed at $\pi$ originating from decays in flight, which makes it difficult to estimate this background. Adding the $\delta \phi(\mu, u)$ distribution from decays in flight candidates from the impact parameter sidebands to the $W \rightarrow \mu \nu$ Monte Carlo, we estimate this background to be $(1.0 \pm 1.0) \%$. The $M_{T}$ distribution contains 3116 events and is shown in Figure 7.4. It is obtained from the impact parameter sidebands, which is dominated by decays in flight.

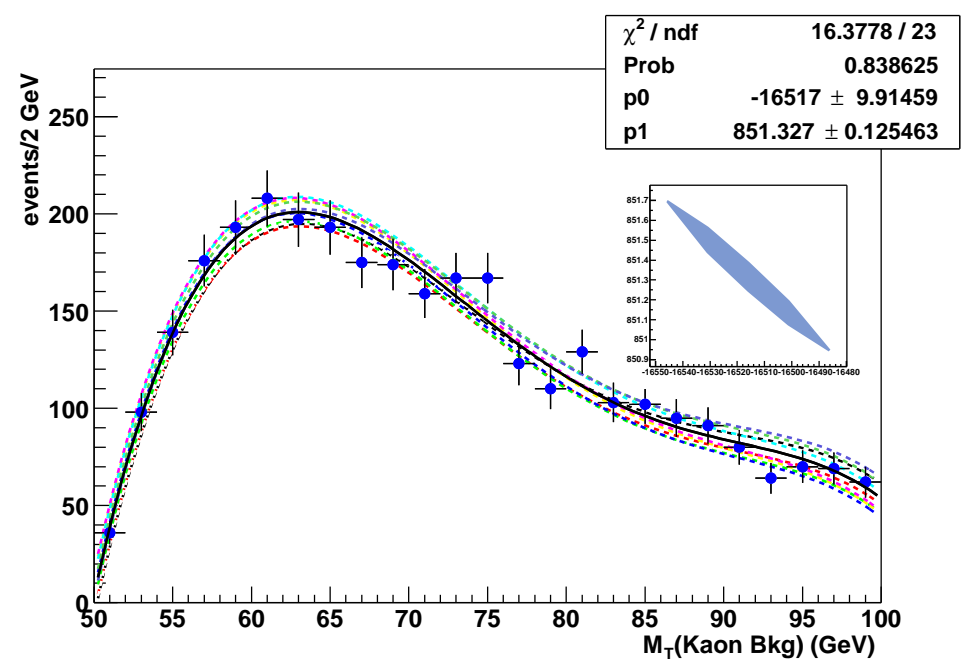

Figure 7.4: $M_{T}$ distribution for kaon background. The best fit to the data and the $3 \sigma$ fitted shape uncertainty are overlaid. 


\subsection{QCD Background}

Events in which real or fake muons from hadronic jets are reconstructed in the detector is one of the more challenging backgrounds. Sometimes real muons are produced in semileptonic decays of hadrons, whereas other times events contain other particles in hadronic jets that are misidentified and reconstructed as a muon. Typically, these events are rejected from our $W$ candidate sample, since we require large $\mathbb{E}_{T}$, but in a small fraction of cases, a significant energy mismeasurement does satisfy the $\mathbb{E}_{T}$ signature of our sample.

The QCD background is estimated using the fact that QCD jet events tend to be non-isolated and have low $\mathbb{E}_{T}$. The first distribution we examine is the $\mathbb{E}_{T}$ spectrum of the $W$ Boson candidates with the $\mathbb{E}_{T}$ cut removed, shown in Figure 7.5. The local maxima at low $\mathbb{E}_{T}$ arises from QCD background events. We model the observed data distribution with $W$ Boson Monte Carlo events with the fractional amounts of $Z \rightarrow \mu \mu$ and $W \rightarrow \tau \nu$ backgrounds added, which will be discussed later in this chapter. The normalization of the QCD shape is varied, added to the $W$ Boson Monte Carlo and the $\chi^{2}$ computed for each. The QCD shapes are obtained from an event sample where the calorimeter isolation fraction (iso) is required to be larger than 0.3. Figure 7.5 shows the $W \rightarrow \mu \nu E_{T}$ distribution with 1.7\% QCD background added, which represents the value from the $\chi^{2}$ minimum. The $W$ Boson candidates are shown in blue, the $W$ Boson Monte Carlo events, with backgrounds added, are shown in red. The purity of the background shape obtained by requiring iso $>0.3$ is not $100 \%$. Using signal Monte Carlo samples, we estimate a $0.3 \%$ signal contamination, reducing the QCD background estimate to $(1.7-0.3=1.4) \%$.

We examine two other distributions where we expect a significant discrimination between QCD and $W \rightarrow \mu \nu$ candidate events, namely, the track isolation and calorimeter isolation ratio distributions. This time the order is reversed and the QCD shapes are obtained from $W$ Boson candidate events with low $\mathbb{E}_{T}$. The normalization of the QCD 


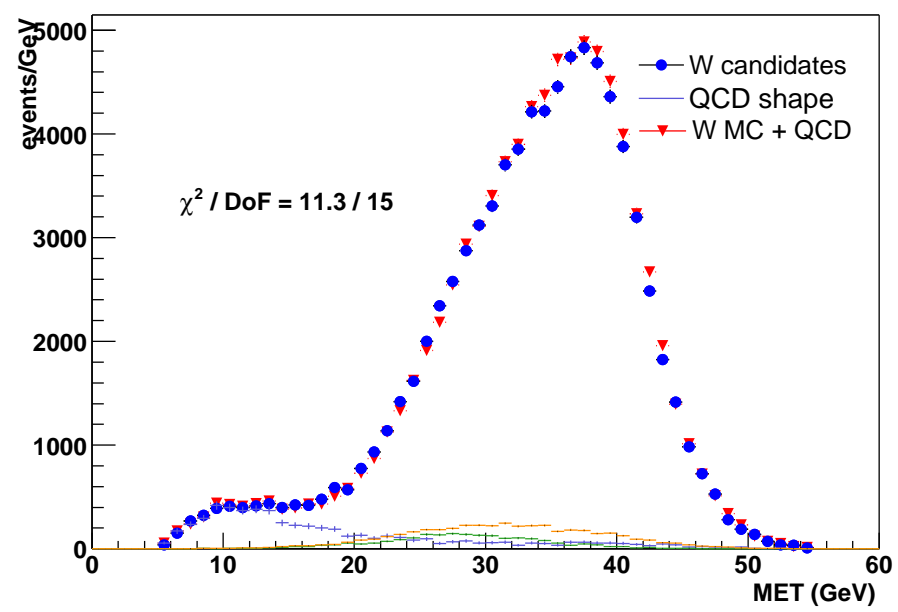

Figure 7.5: $\chi^{2}$ fit for 1.7\% QCD shape added in the $\#_{T}$ distribution.

shape is varied and added to a QCD background free $Z \rightarrow \mu \mu$ data sample to match the calorimeter isolation distributions for the $W$ Boson signal, shown in Figure 7.6. The $\chi^{2}$ is computed between the combined $Z+\mathrm{QCD}$ candidates and the distribution from the $W$ data sample. The distribution for the QCD shape is shown in green, the combined shape from the $Z \rightarrow \mu \mu$ sample and QCD shape is shown in red and the $W$ candidates are represented by the blue points. Figure 7.6 shows the calorimeter isolation ratio where

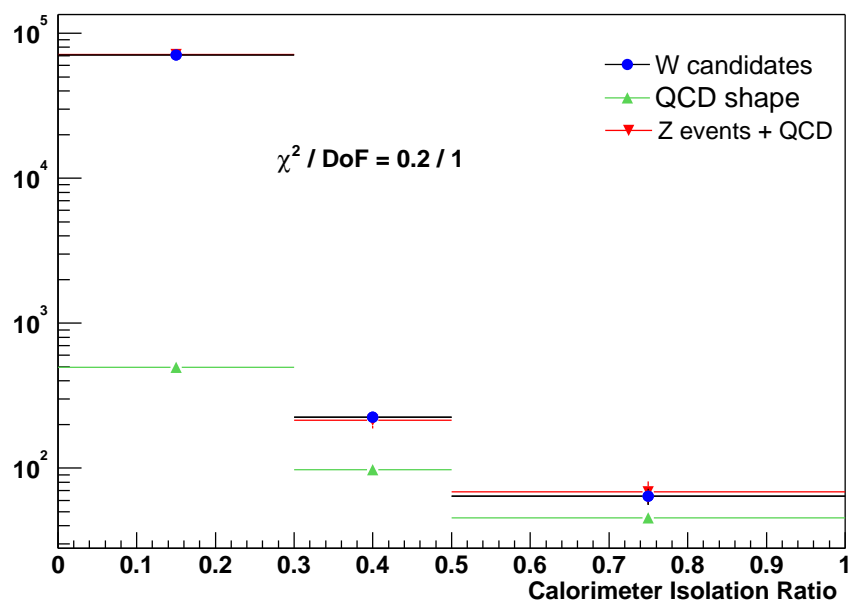

Figure 7.6: $\chi^{2}$ fit for $0.9 \%$ QCD shape added to calorimeter isolation ratio distribution. 
0.9\% QCD background is added, resulting from the $\chi^{2}$ minimization. Applying the same technique using the track isolation ratio rather than the calorimeter isolation ratio gives an estimate of $0.5 \%$. We estimate a total QCD background of $(0.9 \pm 0.5) \%$, using the value obtained from the fit to the calorimeter isolation ratio as the central value and the spread of the two other estimates as the uncertainty.

The transverse mass QCD distribution is obtained by inverting the $\mathrm{E}_{e m}, \mathrm{E}_{\text {had }}, \Delta x$ stub matching cuts and by requiring that the isolation fraction be greater than 0.25 . If three out of four of these conditions in addition to the other $W$ selection cuts are met, the event is included in the QCD shape. There are 230 events that pass these requirements and the transvers mass distribution is shown in Figure 7.7.

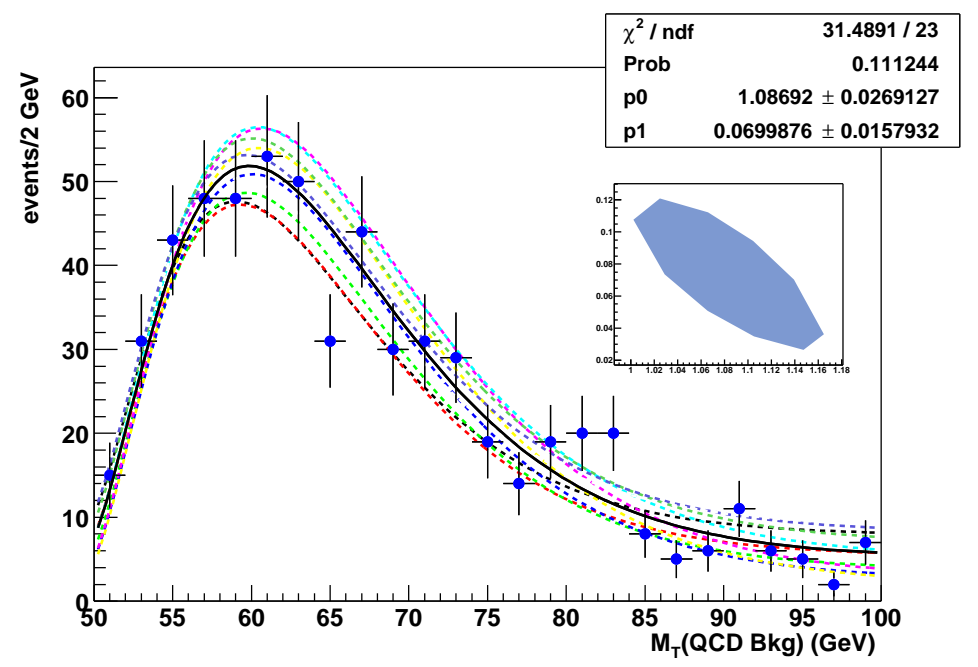

Figure 7.7: $M_{T}$ distribution for QCD background. The best fit to the data and the $3 \sigma$ fitted shape uncertainty are overlaid.

\subsection{Backgrounds from $Z \rightarrow \mu \mu$ and $W \rightarrow \tau \nu$}

When the second lepton in a $Z \rightarrow \mu \mu$ event is not reconstructed in the detector, the event looks just like a $W$ Boson event. When a $W$ Boson decays to a tau lepton and the tau then decays into a muon and a neutrino, it is also indistinguishable from the signal. 
Both of these electroweak background processes are well understood and large Monte Carlo samples generated with PYTHIA and simulated with cdfSim are used to estimate their size and shape. In order to reduce a large fraction of the $Z \rightarrow \mu \mu$ background, events which have two isolated, minimum ionizing high $p_{T}$ tracks with opposite charges are rejected according to the $Z$ rejection defined in the event selection in section 4.2.1. Figure 7.8 shows the rejected events with $Z \rightarrow \mu \mu$ Monte Carlo overlaid. The two

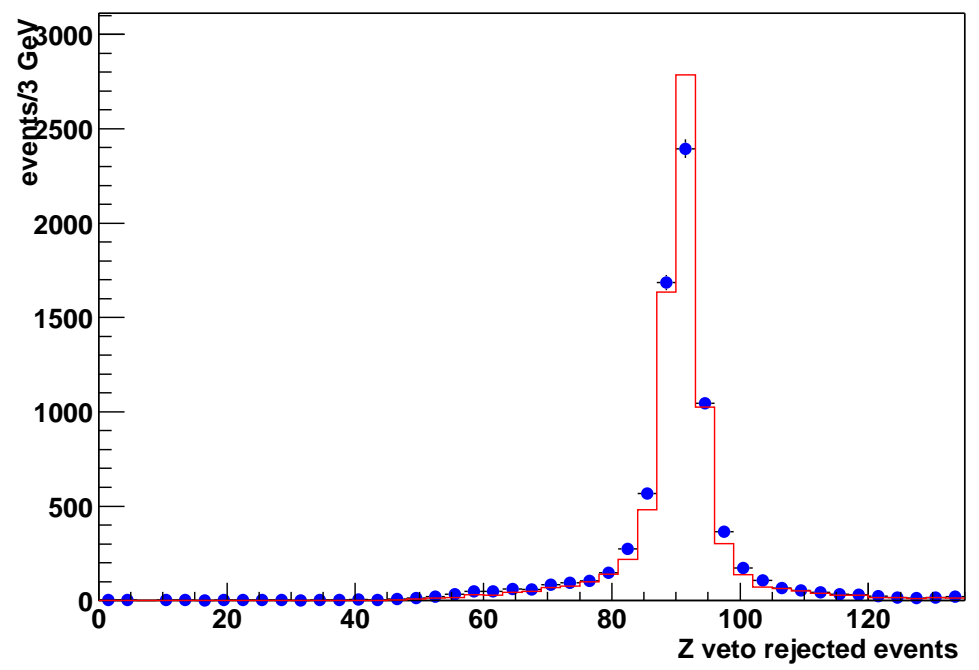

Figure 7.8: Events rejected by $Z$ veto with $Z \rightarrow \mu \mu$ Monte Carlo overlaid.

distributions show reasonable agreement. To evaluate how many $W$ Boson events are rejected in this way we try to select fake $Z \rightarrow \mu \mu$ candidates in $W \rightarrow \mu \nu$ Monte Carlo. We find only one such event in a Monte Carlo sample comparable to the data statistics.

To estimate the $Z \rightarrow \mu \mu$ and $W \rightarrow \tau \nu$ backgrounds we use large Monte Carlo samples generated with PYTHIA that have been simulated with the full CDF detector simulation. We apply our $W \rightarrow \mu \nu$ event selection to both samples as well as to our $W \rightarrow \mu \nu$ signal Monte Carlo sample and compute the individual acceptances for each process. By using Standard Model values for the relative production rates of the three processes and these acceptance values, one can calculate the relative contribution from each process.

The Standard Model predicts equivalent production cross-sections for $W \rightarrow \mu \nu$ and 
$W \rightarrow \tau \nu$, while the $W \rightarrow \mu \nu$ and $Z \rightarrow \mu \mu$ production cross-sections are related by a factor $R$. In order to extract the relative contributions from $Z \rightarrow \mu \mu$ events compared to our $W \rightarrow \mu \nu$ candidate samples, an input value for $R$ is required. A NNLO calculation finds $R=10.67 \pm 0.15$ at $\sqrt{s}=1.96 \mathrm{TeV}$ [48]-[52]. The theoretical uncertainty of $R$ is combined with an experimental uncertainty based on the Run 1 measurement [53] resulting in $R=10.67 \pm 0.45$. Table 7.2 shows the calculation of the relative acceptances, the applied

\begin{tabular}{llll}
\hline & $W \rightarrow \mu \nu \mathrm{MC}$ & $Z \rightarrow \mu \mu \mathrm{MC}$ & $W \rightarrow \tau \nu \mathrm{MC}$ \\
\hline Generated MC events & 474450 & 93681 & 466453 \\
Passing all cuts & 72527 & 8760 & 1448 \\
Acceptance & $15.29 \pm 0.06$ & $9.35 \pm 0.10$ & $0.31 \pm 0.01$ \\
Correction for R & & $0.88 \pm 0.04$ & \\
Acceptance Fraction & $92.80 \pm 0.51$ & $5.32 \pm 0.21$ & $1.88 \pm 0.05$ \\
\hline Background in \% & & $5.20 \pm 0.21$ & $1.84 \pm 0.05$ \\
\hline
\end{tabular}

Table 7.2: Relative acceptances for $W \rightarrow \mu \nu, Z \rightarrow \mu \mu$ and $W \rightarrow \tau \nu$

correction to the $Z \rightarrow \mu \mu$ acceptance and the fractions of each individual process to the combined acceptance. The background fraction from $Z \rightarrow \mu \mu$ and $W \rightarrow \tau \nu$ events shown in the last line of Table 7.2 are then obtained by correcting for QCD, decay in flight and cosmic background events which were not included in the combined acceptance of the three processes. Figures 7.9 and 7.10 show the transverse mass distributions for the $Z \rightarrow \mu \mu$ and $W \rightarrow \tau \nu$ backgrounds obtained from the corresponding PYTHIA Monte Carlo samples. The transverse mass distribution contains 8760 events for the $Z \rightarrow \mu \mu$ background and 1448 events for the $W \rightarrow \tau \nu$ background.

In order to obtain the systematic uncertainty on the $W$ Boson mass due to the backgrounds, a high statistics Monte Carlo template and Monte Carlo data file are generated with the fast detector simulation. The data file is then fitted applying different background sizes or varied background shapes to the Monte Carlo template. Figure 7.11 shows 


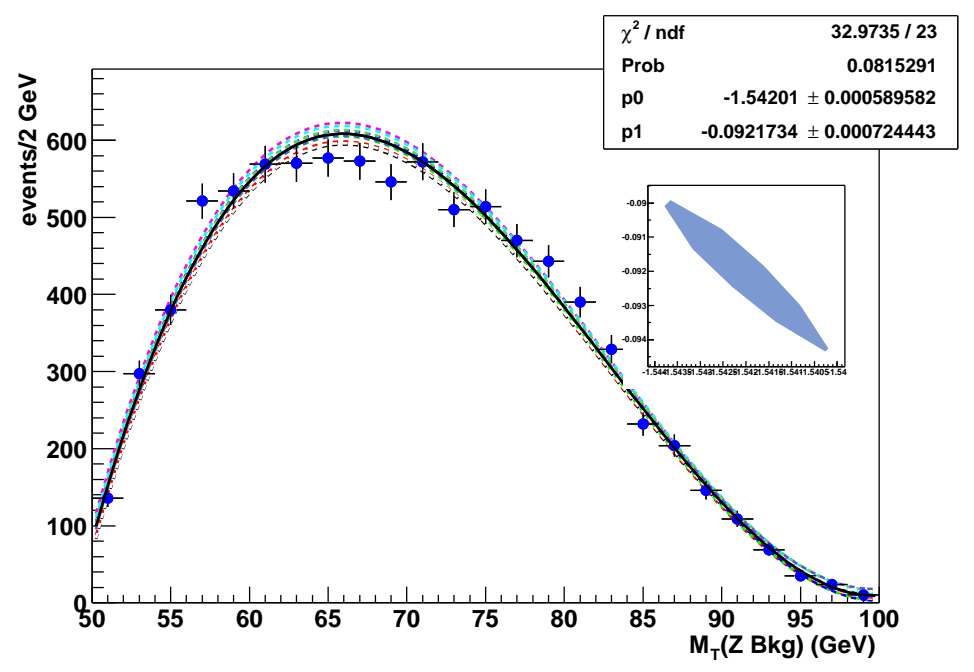

Figure 7.9: $M_{T}$ distribution for $Z \rightarrow \mu \mu$ background. The best fit to the data and the $3 \sigma$ fitted shape uncertainty are overlaid.

the $M_{T}$ distributions, for all backgrounds and total background overlaid. To estimate the systematic uncertainty on the $W$ mass due to the background size uncertainties, the backgrounds are varied by their uncertainties in the Monte Carlo template. The fitted kinematic shape uncertainty is estimated by fitting different shapes obtained from the

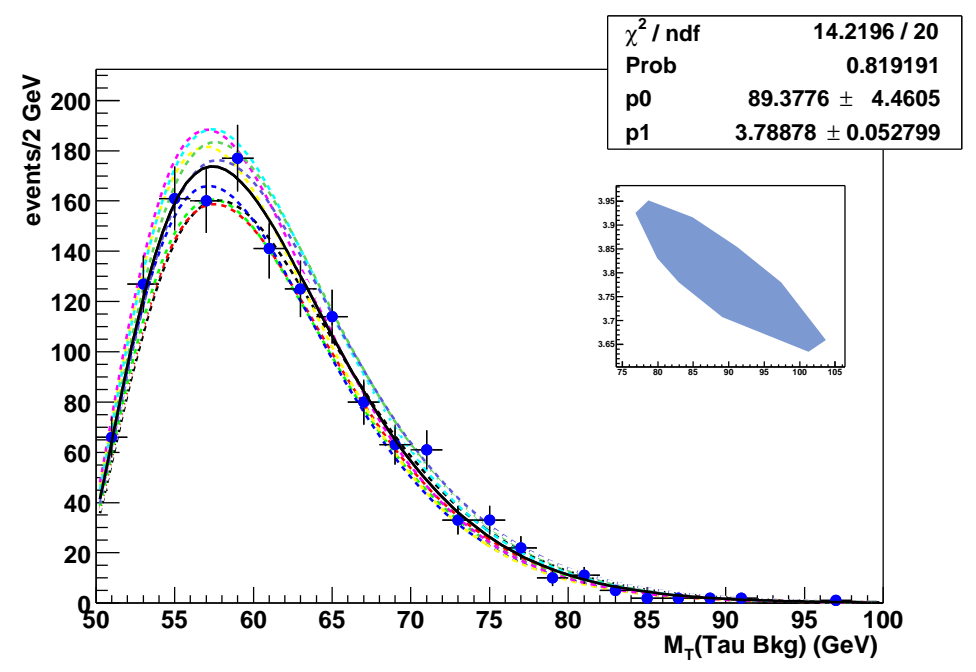

Figure 7.10: $M_{T}$ distribution for $W \rightarrow \tau \nu$ background. The best fit to the data and the $3 \sigma$ fitted shape uncertainty are overlaid. 


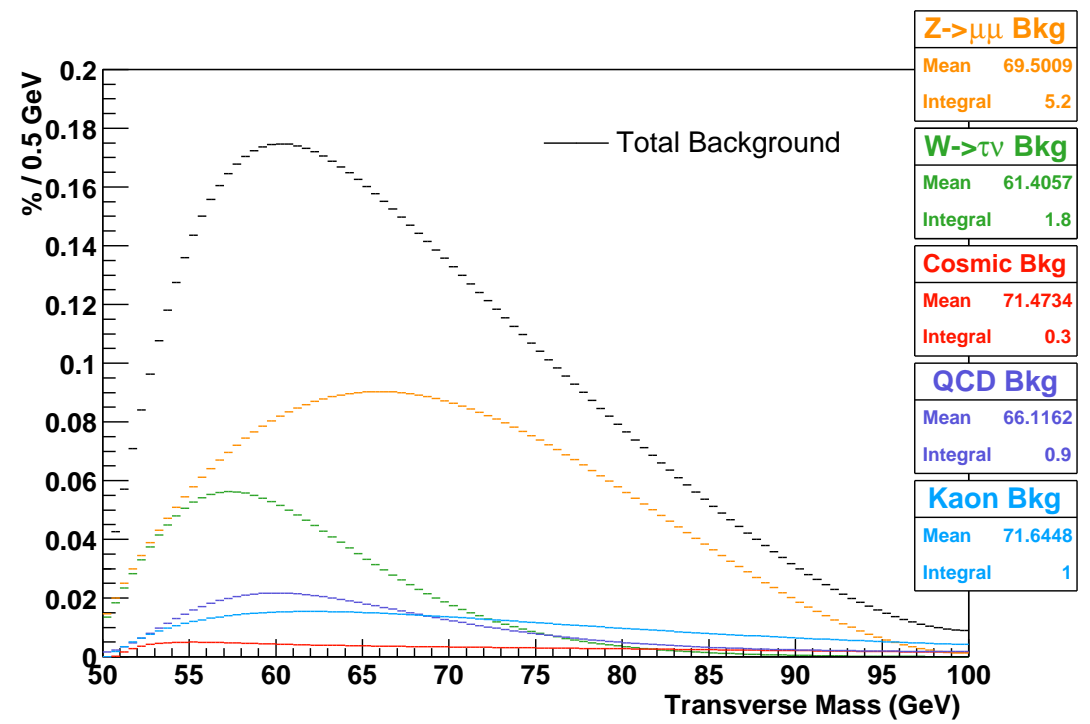

Figure 7.11: $M_{T}$ distributions for all backgrounds and total background overlaid.

\begin{tabular}{lccc}
\hline Background & Size $[\%]$ & $\Delta M_{W}\left[\mathrm{MeV} / \mathrm{c}^{2}\right]($ size $)$ & $\Delta M_{W}\left[\mathrm{MeV} / \mathrm{c}^{2}\right]$ (shape) \\
\hline $\mathrm{Z} \rightarrow \mu \mu$ & $5.2 \pm 0.2$ & 2 & 5 \\
$\mathrm{~W} \rightarrow \tau \nu$ & $1.8 \pm 0.1$ & 4 & 1 \\
QCD & $0.9 \pm 0.5$ & 12 & 7 \\
Kaon & $1.0 \pm 1.0$ & 8 & 2 \\
Cosmic Rays & $0.3 \pm 0.1$ & 1 & 0 \\
\hline
\end{tabular}

Table 7.3: Systematic uncertainty on $W$ mass due to background uncertainty.

$3 \sigma$ contour of the fit parameters. These shape variations are applied to the Monte Carlo template and a fit value is obtained for each. The largest variations adjusted to $1 \sigma$ are taken as the shape uncertainty. The systematic variations are listed in Table 7.3. In summary, the systematic uncertainty on the $W$ mass due to backgrounds is $18 \mathrm{MeV} / \mathrm{c}^{2}$. 


\section{Chapter 8}

\section{Hadronic Recoil Measurement}

The transverse mass distribution used for the $W$ Boson mass measurement is reconstructed using the transverse momentum of the muon and the neutrino. The transverse momentum of the neutrino is inferred from the charged muon and the recoil energy $u_{T}$ (see Figure 5.17). This chapter describes the measurement of $u_{T}$ and the tuning of the simulation outlined in section 5.2.4. Since the $W$ and $Z$ share a common production mechanism and are close in mass, the recoil model for $W$ Bosons is constrained by using $Z \rightarrow \mu \mu$ decays. For $Z \rightarrow \mu \mu$ events, we can measure the transverse momentum $u_{T}$ in the same way as for the $W \rightarrow \mu \nu$ events. By comparing $p_{T}^{\mu \mu}$, which is well measured,

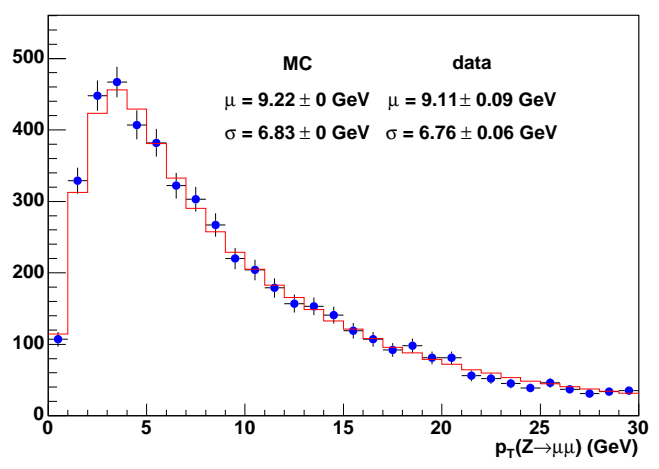

Figure 8.1: Distribution of $p_{T}(\mu \mu)$ in $Z \rightarrow$ $\mu \mu$ events.

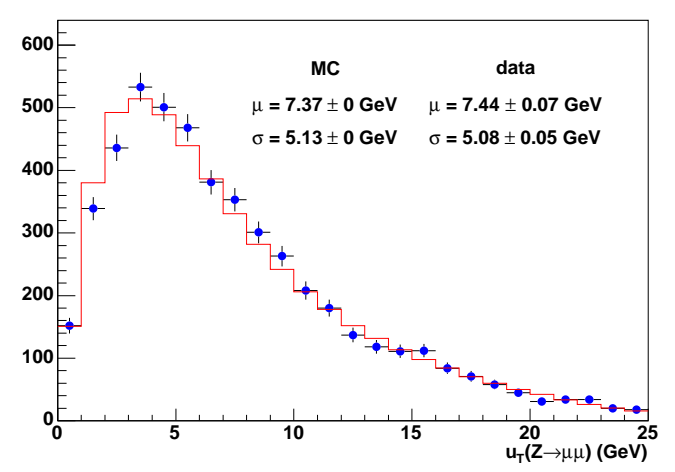

Figure 8.2: Distribution of $u_{T}$ in $Z \rightarrow \mu \mu$ events. 
and $u_{T}$, we calibrate the recoil response relative to the charged track response. These distributions for $Z \rightarrow \mu \mu$ events are shown in Figure 8.1 and Figure $8.2^{1}$. The recoil momentum is carried by many particles, mostly hadrons, with a wide momentum spectrum. Since the response of the calorimeter to hadrons is slightly nonlinear at low energies and the recoil particles see a reduced response at module boundaries, we expect a momentum dependent response function with values below unity.

\subsection{Recoil Response}

For the purpose of modeling the recoil, the transverse momentum balance for $Z \rightarrow \mu \mu$ events is computed between the recoil as reconstructed by the calorimeter $\left(u_{T}\right)$ and that reconstructed by the two muons $\left(p_{T}^{\mu \mu}\right)$. It is natural to define the $p_{T}$-balance along two directions. One is projected onto the bisector axis, $\eta$, of the two muon candidates, which is mainly sensitive to the recoil response, and the other perpendicular to it, $\xi$, which is mainly sensitive to the recoil resolution. Figure 8.3 shows the different components.

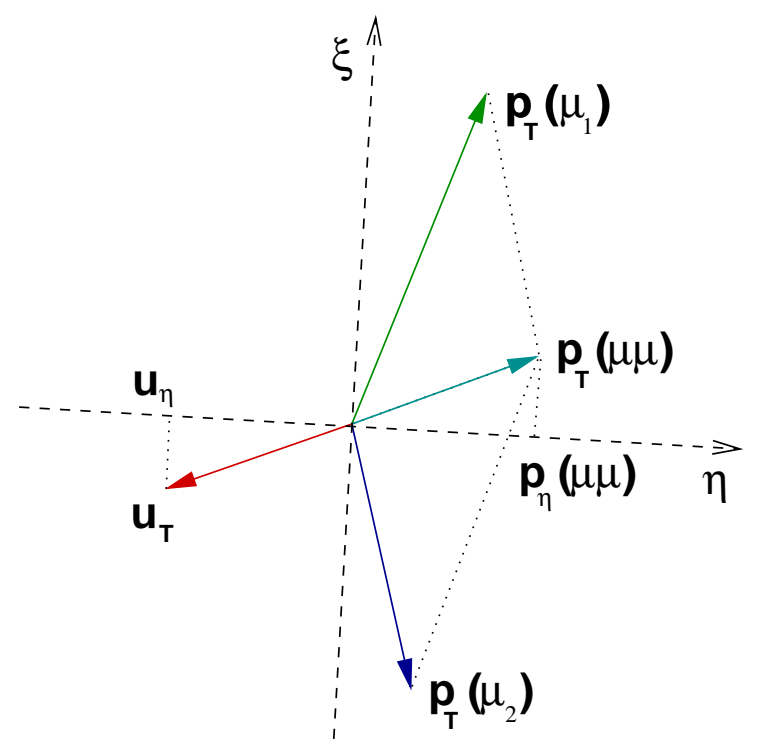

Figure 8.3: Illustration of momentum vectors in the transverse plane for $Z \rightarrow \mu \mu$ events.

\footnotetext{
${ }^{1}$ An uncertainty of zero indicates, that the uncertainty is $<0.01$ and therefore neglegible.
} 
In order to tune the scale parameters $a, b$ and $c$ of the response function, introduced in section 5.2.4, the mean value of the $p_{T}$-balance in the $\eta$ direction is compared between data and simulation, as shown in Figure 8.4. The parameters are adjusted by minimizing the $\chi^{2}$ to best match the data distribution .

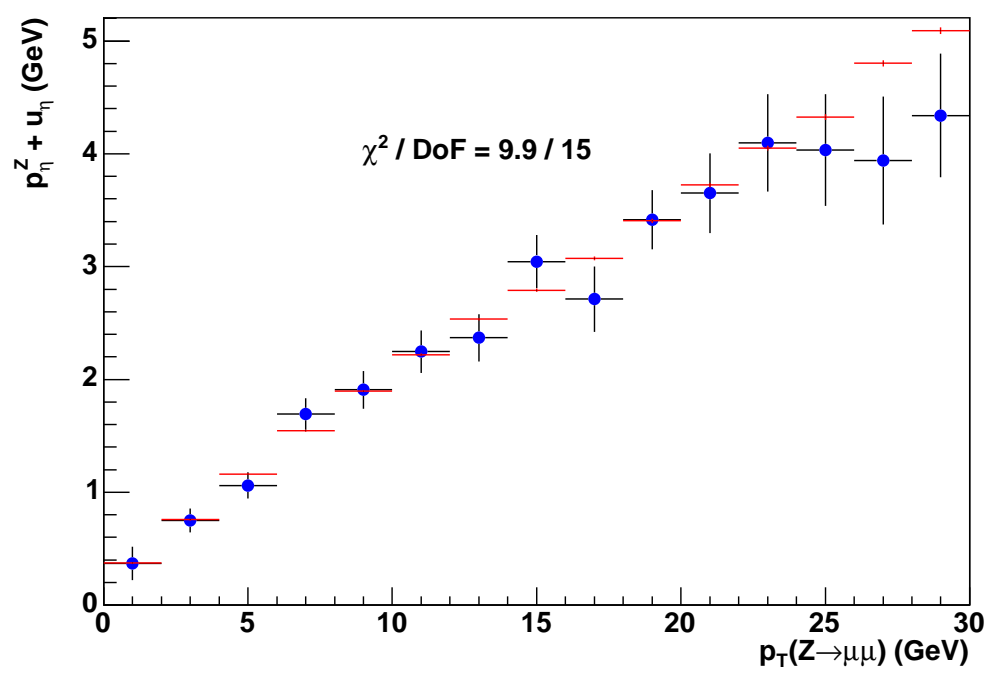

Figure 8.4: The mean value of the $p_{T}$-balance in the $\eta$ direction in $Z \rightarrow \mu \mu$ events. The dots represent the data, the histogram the simulation.

\subsection{Recoil Resolution}

The resolution parameters are measured by comparing the root mean square $(\sigma)$ of the $p_{T}$-balance in the $\eta$ direction as a function of $p_{T}^{\mu \mu}$. This is shown in Figure 8.5. The parameter sampling resolution term, $s_{\text {had }}$, is sensitive to the slope of this comparison. The intercept of the plot is sensitive to the amount of the soft recoil component, originating from proton remnants and multiple interactions. 


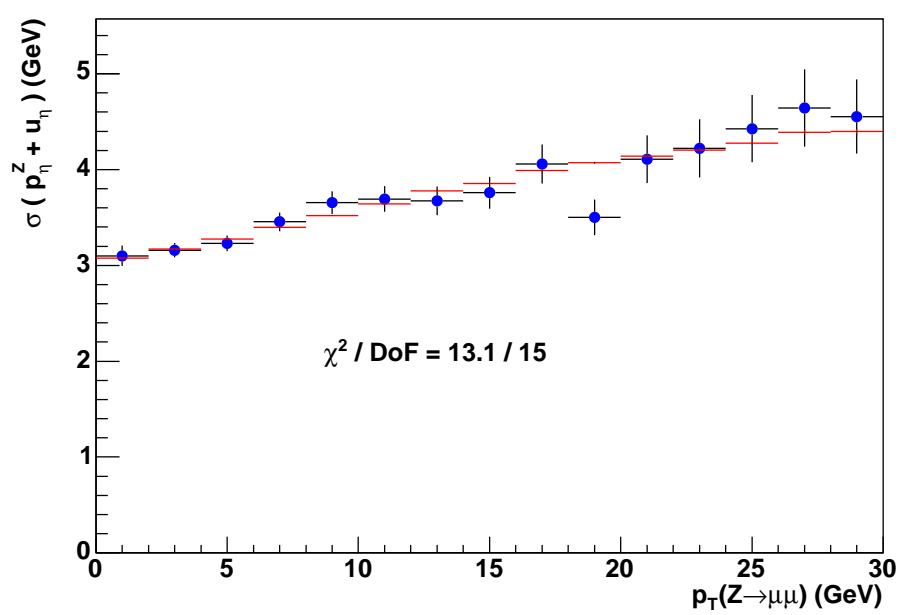

Figure 8.5: The rms value of the $p_{T}$-balance in the $\eta$ direction in $Z \rightarrow \mu \mu$ events.

\subsection{W Recoil}

The ultimate test of the recoil model is a comparison of the recoil quantities for the $W$ Boson with the simulation. Figure 8.6 shows the $W$ Boson $u_{T}$ distribution comparison and Figure 8.7 the angular difference between the recoil and the muon in the $r$ - $\phi$ plane. As has been described in chapter 7, the $W$ Boson signal has several background contributions, which are included in the simulation for these comparisons. As in the $Z$ Boson
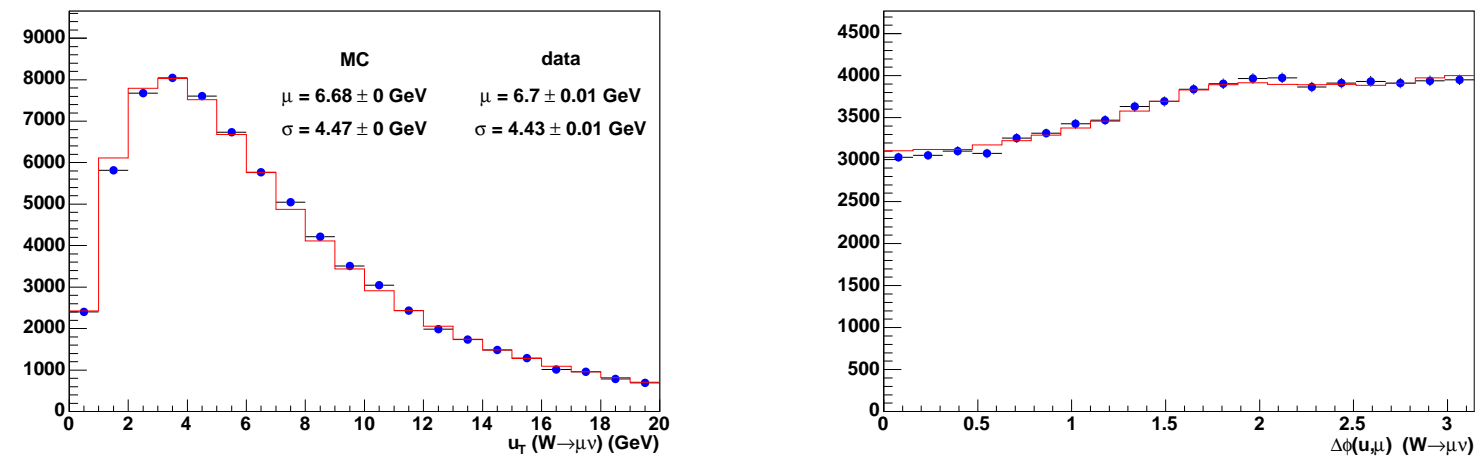

Figure 8.6: Distribution of $u_{T}$ in $W \rightarrow$ $\mu \nu$ events. Background contributions are included in the simulated distribution.
Figure 8.7: Distribution of $\Delta \phi(\mu, u)$ in $W \rightarrow \mu \nu$ events. Background contributions are included in the simulation. 
case, we split the $W$ recoil into two components. One parallel to the muon $\left(u_{\|}\right)$and one perpendicular to it $\left(u_{\perp}\right)$. The components are illustrated in Figure 5.17. Figure 8.8 and Figure 8.9 show the $u_{\|}$and $u_{\perp}$ distributions for the $W$ candidates.

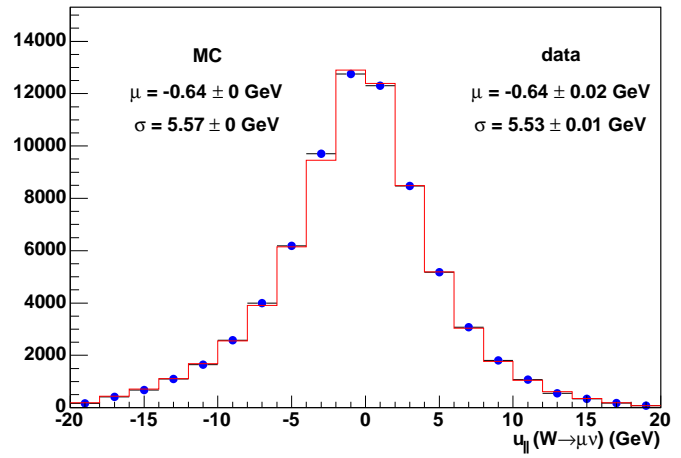

Figure 8.8: Distribution of $u_{\|}$in $W \rightarrow$ $\mu \nu$ events. Background contributions are included in the simulated distribution.

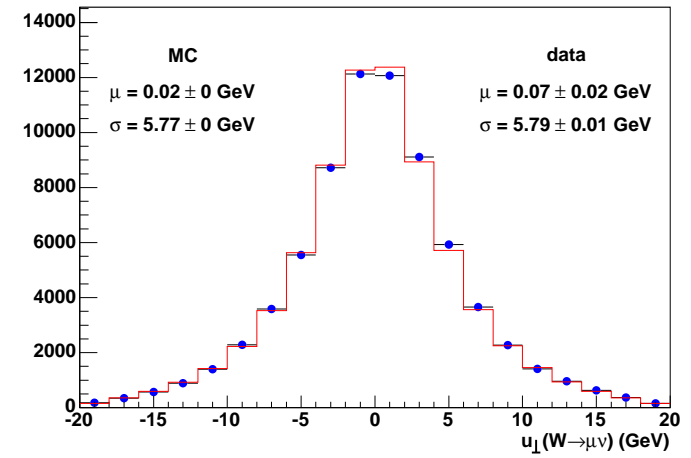

Figure 8.9: Distribution of $u_{\perp}$ in $W \rightarrow$ $\mu \nu$ events. Background contributions are included in the simulated distribution.

The systematic uncertainty associated with the recoil model consists of two parts. For the recoil response $R$, the three parameters are varied within their uncertainties to alter the $\chi^{2}$ in the $\eta$ balance by unity, which alters the fitted $W$ Boson mass by $20 \mathrm{MeV} / c^{2}$. For the recoil resolution, the parameter $s_{\text {had }}$ is varied by its uncertainty, resulting in an additional $20 \mathrm{MeV} / c^{2}$ uncertainty on the $W$ Boson mass. The uncertainty in modeling the soft underlying event resolution parametrized using minimum bias data contributes an additional $14 \mathrm{MeV} / c^{2}$. Combining the uncertainty arising from the recoil response and resolution, we estimate an overall systematic uncertainty from the recoil model of 32 $\mathrm{MeV} / c^{2}$ on the $W$ Boson mass. 


\section{Chapter 9}

\section{Result}

The $W$ mass is extracted by performing a binned maximum-likelihood fit to the transverse mass, $m_{T}$, distribution. The template includes all $W$ production and detector effects described in the previous chapters. The templates are generated with input masses between 80 and $81 \mathrm{GeV} / c^{2}$, in steps of $2.5 \mathrm{MeV} / c^{2}$. Figure 9.1 shows the templates.

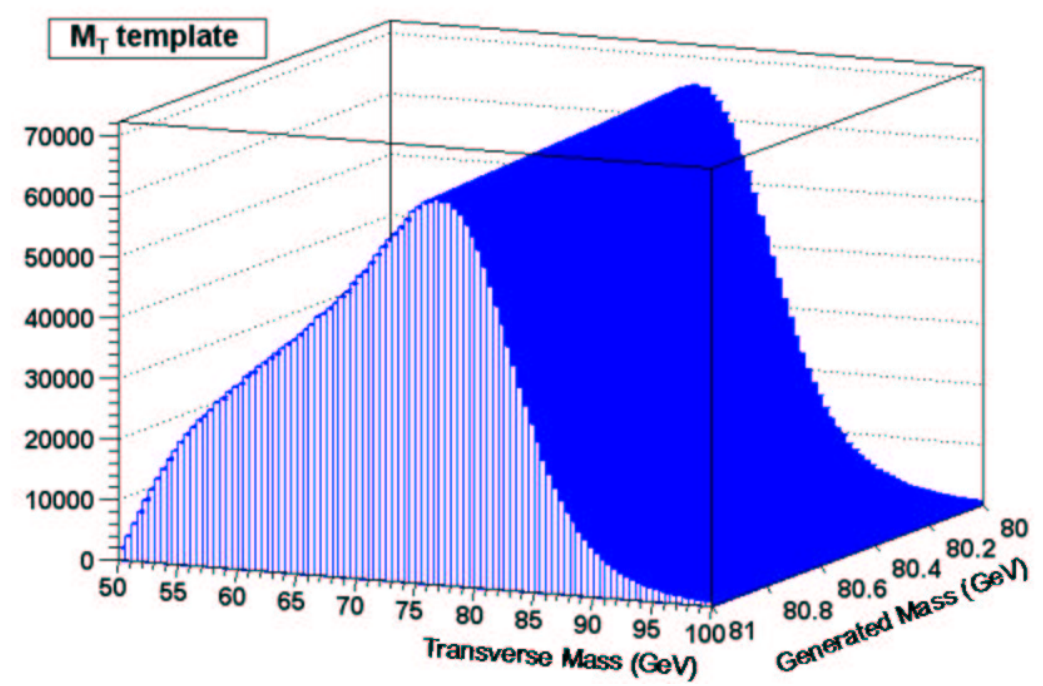

Figure 9.1: Transverse mass template used to extract the $W$ Boson mass.

Background distributions are added to the simulated distributions with their nominal shape normalizations. The sum of the simulated signal and backgrounds are normalized 
so that their integral in the specified fit region equals the integral of the data distribution in the same region. Simulation templates are used to give the expected number of events in each bin and the Poisson probability of the data in each bin is computed. The total likelihood is defined as the product of the Poisson probabilities over all bins in the fit region. The total likelihood is maximized as a function of the true mass associated with the simulation template.

This chapter will provide a summary of the result of the fit to the $W$ transverse mass distribution in the muon decay channel. Included is a section summarizing all the systematic uncertainties that have been described in the previous chapters.

\subsection{Fit Results}

In order not to bias the $W$ Boson mass analysis during its study, the fit result (the actual fit mass value) was blinded until the analysis was completed. For this purpose, a single random offset from a flat distribution between $\pm 100 \mathrm{MeV} / \mathrm{c}^{2}$ was generated. This offset was put in a file from which read access was removed.

After the analysis was complete and all cross-checks were performed, the mass offset was removed. Figure 9.2 shows the fit to the transverse mass. The fit region is $60<$ $m_{T}<90 \mathrm{GeV} / \mathrm{c}^{2}$. The distribution shows good agreement with the simulation and the fitted $W$ Boson mass value is:

$$
M_{W}=80408 \pm 50 \mathrm{MeV} / \mathrm{c}^{2}
$$

where the quoted uncertainty is statistical only. Once we have discussed the systematic uncertainties, we can proceed to compare the results to previously measured $W$ Boson mass values. 


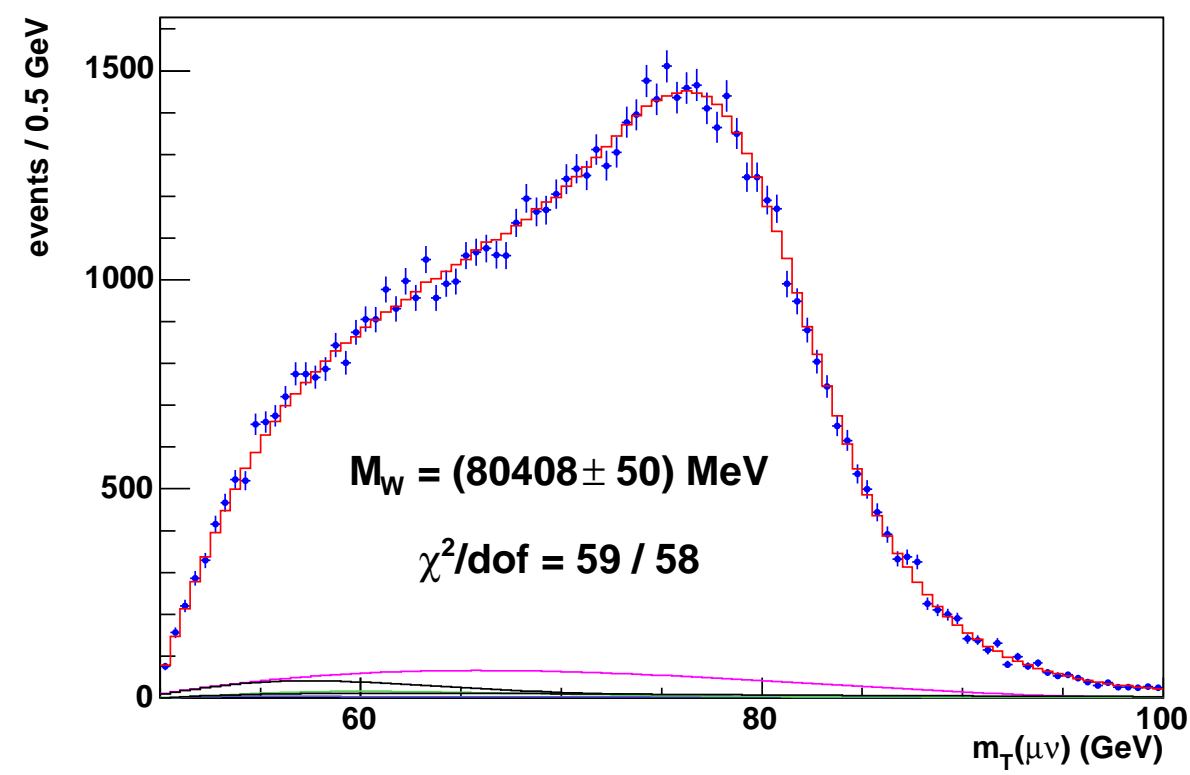

Figure 9.2: The $W \rightarrow \mu \nu$ transverse mass fit.

\subsection{Summary of Systematic Uncertainties}

This section summarizes the systematic uncertainties resulting from the study of the various inputs to the kinematic lineshape model as described in the previous chapters. Table 9.1 gives a summary of all systematic uncertainties. For comparison, the uncertainties from the CDF measurement in the previous collider run (Run 1b) which used $80 \mathrm{pb}^{-1}$ of data are also included. In some cases there are uncertainties which we have treated separately which were combined in the Run $1 \mathrm{~b}$ result.

The momentum scale uncertainty includes $18 \mathrm{MeV} / \mathrm{c}^{2}$ from the scale and $20 \mathrm{MeV} / \mathrm{c}^{2}$ from the curvature corrections. This results in a $27 \mathrm{MeV} / \mathrm{c}^{2}$ uncertainty as described in chapter 6 . The reduced uncertainty compared to Run $1 \mathrm{~b}$ results from the fact that the quarkonium resonances were used instead of the $Z$ Boson to set the momentum scale.

The resolution of the muon momentum measurement changes the slope of the Jacobian edge resulting in an apparent mass shift. A constraint on this resolution comes from the COT hit resolution measured in $Z \rightarrow \mu \mu$ events as described in section 6.4. To 
reduce the sensitivity to the finite statistics of even very large Monte Carlo samples, the resolution in the lineshape simulation is varied up and down by twice the uncertainties quoted on the respective resolutions. The apparent mass shifts from Monte Carlo simulations are then divided by four to get the effective $1 \sigma$ systematics due to charge lepton resolution. This results in a systematic uncertainty of $12 \mathrm{MeV} / \mathrm{c}^{2}$.

\begin{tabular}{ccc} 
Systematic $\left(\mathrm{MeV} / \mathrm{c}^{2}\right)$ & Run 2 & Run $1 \mathrm{~b}$ \\
\hline Momentum Scale & 27 & 85 \\
Momentum Resolution & 12 & 20 \\
Recoil Scale & 20 & 35 \\
Recoil Resolution & 24 & " \\
$u_{\|}$efficiency & 7 & 18 \\
Muon Removal & 10 & " \\
Backgrounds & 18 & 25 \\
$p_{T}(W)$ model $\left(g_{2}, g_{3}\right)$ & 14 & 20 \\
Parton Distributions & 15 & 15 \\
QED radiation & 20 & 10 \\
$\Gamma_{W}$ & 12 & \\
\hline Total & 57 & 103
\end{tabular}

Table 9.1: Table of systematic uncertainties for the transverse mass fit. When comparing to the Run 1b result, some uncertainties were combined in the Run 1b measurement.

The uncertainties on the recoil model parameters are varied in the Monte Carlo simulation described in section 5.2.4. We take the resulting shifts in mass to get the systematics quoted in the table.

The systematic uncertainty from muon identification inefficiencies, described in sec- 
tion 5.2.6, is estimated by varying the fit parameter by its uncertainty. This results in a $7 \mathrm{MeV} / c^{2}$ uncertainty on the $W$ Boson mass.

We estimate that there is a $10 \mathrm{MeV} / c^{2}$ systematic uncertainty in the underlying event energy that is removed when we remove the muon candidate towers in the recoil measurement (see section 5.2.5).

Backgrounds are discussed in chapter 7. The fitted shapes and normalizations of the various background constituents are varied by their uncertainties. The QCD and decay in flight backgrounds are the least well understood and contribute by far the largest systematic uncertainty. We end up with a systematic uncertainty of $18 \mathrm{MeV} / \mathrm{c}^{2}$.

In section 5.1.2 we investigated the influence of the non-perturbative, phenomenological parameters which control the low $p_{t}(W)$ shape in the generation of $W$ Bosons with RESBOS. Despite the generous ranges over which these parameters were varied, we conclude that the systematic uncertainty is $14 \mathrm{MeV} / \mathrm{c}^{2}$. This does not cover the shape of the $p_{t}(W)$ distribution for higher momentum $W$ Bosons. This part of the spectrum is much more sensitive to the response of the CDF detector to the underlying event. The systematics for these effects are included in our investigation of the recoil response model.

Section 5.1.2 also discussed the variation of our lineshape due to different choices of parton distribution functions. The full CTEQ6M error PDF set was used, and in conclusion the transverse mass distribution was sensitive at the $15 \mathrm{MeV} / \mathrm{c}^{2}$ level. A cross-check with a recent MRST PDF showed this to be a reasonable level of uncertainty.

Various effects of QED radiation on the lineshape were investigated. We conclude that the corresponding systematic uncertainty is $20 \mathrm{MeV} / \mathrm{c}^{2}$ with the largest part coming from unmodelled higher-order effects.

The fitting templates are generated with $\Gamma_{W}=2.12 \mathrm{GeV} / \mathrm{c}^{2}$. Direct measurements of this width give a world average uncertainty of $70 \mathrm{MeV} / \mathrm{c}^{2}$ which more than covers the indirect width of the $W$ Boson which is $2.09 \mathrm{GeV} / \mathrm{c}^{2}$. This $70 \mathrm{MeV} / \mathrm{c}^{2}$ uncertainty on the width translates into a $12 \mathrm{MeV} / \mathrm{c}^{2}$ uncertainty on the $W$ mass as it distorts the Jacobian 
edge in a similar way to the COT hit resolution.

Adding all individual systematic uncertainties we obtain a total systematic uncertainty of $57 \mathrm{MeV} / \mathrm{c}^{2}$ on the $W$ Boson mass for this measurement. This is an improvement of almost a factor of two relative to the measurement in the previous collider run, where a systematic uncertainty of $103 \mathrm{MeV} / \mathrm{c}^{2}$ was found.

\subsection{Cross-Checks}

We have divided the $W$ Boson candidate sample into sub-samples and for each of these performed the $W$ Boson mass fits. The results are listed in Table 9.2. The sample

\begin{tabular}{cc} 
Sub-sample & $m_{W}$ \\
\hline positive charge & $+112 \pm 69$ \\
negative charge & $-110 \pm 69$ \\
early $($ run $<161000)$ & $-120 \pm 88$ \\
late $($ run $>161000)$ & $+62 \pm 64$ \\
\hline
\end{tabular}

Table 9.2: Fitted $W$ Boson mass shifts (in $\mathrm{MeV} / \mathrm{c}^{2}$ ) for sub-divisions of the data.

separation with respect to the charge of the decay muon checks for a dependency that could be introduced by wire misalignments in the tracking chamber. We do correct for residual misalignments by applying a curvature correction to the muon track as described in section 6.1. Therefore, the charge separated fits also check the effectiveness of the curvature correction. The second separation of the data sample investigates whether there might be a dependence on the run ranges used, which could possibly originate from the steady increase in instantaneous luminosity of the Tevatron collider, or from the aging of the CDF detector components. No statistically significant variation in the fitted $W$ Boson mass is observed in either of the two data sub-samples. 
We show the signed $\chi$ distributions from the transverse mass fit in Figure 9.3. The $\chi$

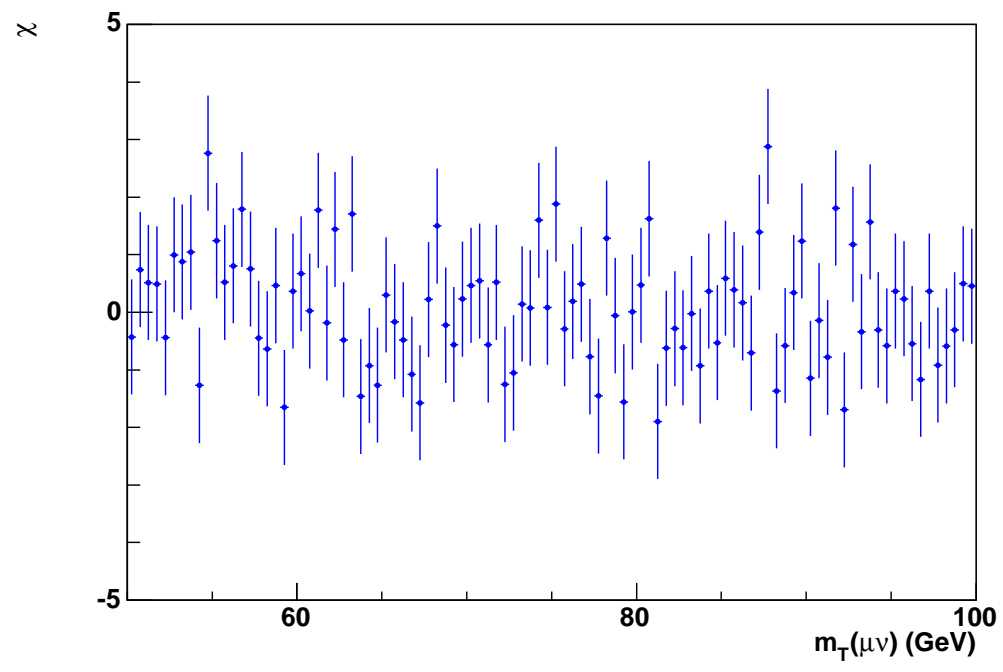

Figure 9.3: Normalized $\chi$ distribution for the $W$ mass fit.

distribution is the difference of the number of entries of data and simulation in each bin divided by the error. The distribution shows no bias.

\subsection{Comparison to Previous Results}

Table 9.3 shows how this result compares to the other measurements that are currently part of the Particle Data Group world average. The measurement shows good agreement with the current world average.

One should note that all entries in Table 9.3, apart from the entry for this measurement, are averages of $W$ Boson mass results from multiple $W$ Boson decay channels and two or more experiments. We also note that this measurement is already slightly more precise than the CDF $W$ Boson mass measurement in the previous collider run where both decay channels $W \rightarrow \mu \nu$ and $W \rightarrow e \nu$ were used.

As discussed in chapter 2, together with the mass of the top quark, the $W$ Boson mass constrains the mass of the Higgs Boson. The current world average $W$ Boson mass 


\begin{tabular}{cc} 
Experiment & Mass $\left(\mathrm{MeV} / \mathrm{c}^{2}\right)$ \\
L3 & $80376 \pm 77$ \\
DELPHI & $80404 \pm 74$ \\
OPAL & $80490 \pm 65$ \\
ALEPH & $80379 \pm 58$ \\
CDF-I & $80433 \pm 79$ \\
D0-I & $80483 \pm 84$ \\
\hline LEP Average & $80412 \pm 42$ \\
Tevatron Average & $80452 \pm 59$ \\
\hline World Average & $80425 \pm 34$ \\
\hline This Measurement & $80408 \pm 76$ \\
\hline
\end{tabular}

Table 9.3: Current world's best $W$ Boson mass measurements. Note that the LEP experiment results include about $30 \mathrm{MeV} / \mathrm{c}^{2}$ of correlated systematic uncertainty from the LEP beam energy measurement and color recombination effects.

favors a relatively light Higgs Boson. In general the dependence is such that a lower $W$ Boson mass favors a higher Higgs mass, while a high $W$ Boson mass favors a lower Higgs mass. Since this measurement is very close to the current world average, it enforces the prediction of a relatively light Higgs Boson.

\subsection{Outlook}

The $W$ Boson mass measurement in the CDF collaboration is vital and ongoing [54]. A measurement in the electron decay channel using the same $200 \mathrm{pb}^{-1}$ of data is close to completion. Combined with this measurement, a total uncertainty of $\sim 65 \mathrm{MeV} / \mathrm{c}^{2}$ is 
expected. The $W$ Boson mass can also be extracted from fits to the transverse momentum of the charged decay lepton. Analyses using this technique are also ongoing at CDF. The fit to the transverse momentum distribution will be especially interesting for an analysis with an increased dataset in Run 2, when the high luminosity environment will worsen the detector resolution.

So far, the $W$ Boson mass uncertainty has scaled like $\sqrt{N}$, proportional to the statistics that has been used. This was achieved because most systematic uncertainties are determined using data and because a larger statistical sample produces more accurate results. For example, the quarkonium resonances are used to determine the momentum scale and the $Z$ Boson sample to derive the recoil model. However, most uncertainties in the $W$ Boson production and decay model cannot be constrained from data and need more precise input from theoretical calculations. Fortunately, work in this area has started and therefore the associated uncertainty can be expected to decrease.

Figure 9.4 shows a projection of $W$ Boson mass precision as a function of integrated luminosity, assuming a constant theoretical uncertainty of either $20 \mathrm{MeV} / \mathrm{c}^{2}$ (dashed line) or $30 \mathrm{MeV} / \mathrm{c}^{2}$ (full line) which does not scale like $\sqrt{N}$. Previously achieved precisions

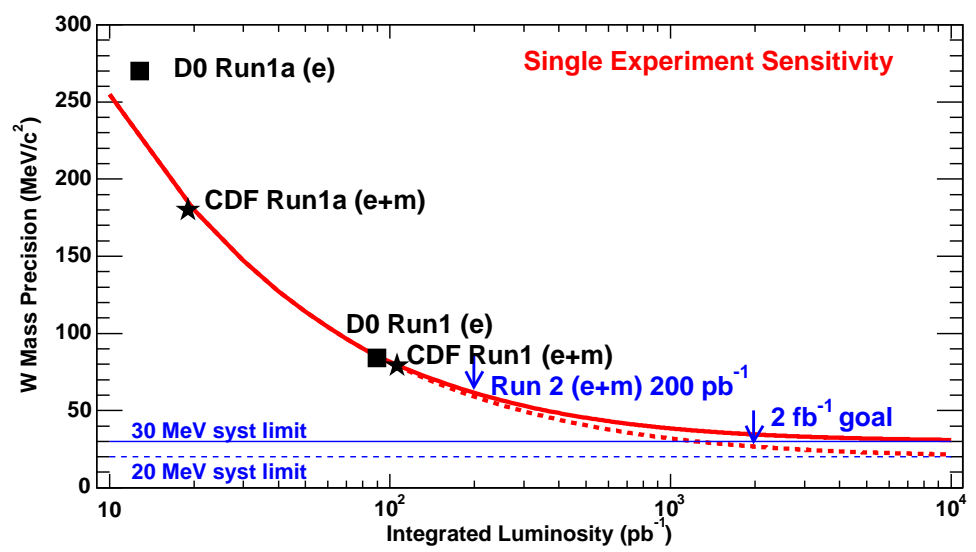

Figure 9.4: Projected $W$ Boson mass precision with integrated luminosity [55]. The black markers shows previously achieved precisions from CDF and DØ, the blue arrows indicate the anticipated precision from CDF for $200 \mathrm{pb}^{-1}$ and $2 \mathrm{fb}^{-1}$. 
for $W$ Boson mass measurements from $\mathrm{CDF}$ and $\mathrm{D} \varnothing$, the other Tevatron experiment, are indicated by black markers and show good agreement with the assumed statistical scaling. The first red arrow shows the expected $W$ Boson mass uncertainty for the CDF Run 2 measurement with the muon and electron channel combined. Continuing the trend of statistical scaling of the uncertainty, it should be possible to measure the $W$ Boson mass to a precision of approximately $30 \mathrm{MeV} / \mathrm{c}^{2}$ with $2 \mathrm{fb}^{-1}$, indicated by the second red arrow. CDF has already collected about $1 \mathrm{fb}^{-1}$ of data, and is expected to double this dataset within the next year. This is an exciting time to measure the $W$ Boson mass at the Tevatron, with the potential to produce the most precise $W$ Boson mass measurement in the world. 


\section{Bibliography}

[1] S. L. Glashow, Partial-Symmetries of Weak Interactions, Nuclear Physics, 22, 579 (1961).

[2] S. Weinberg, A Model of Leptons, Physical Review Letters, 19(21), 1264 (1967).

[3] A. Salam et al, Electromagnetic and Weak Interactions, Physics Letters, 13, 168 (1964).

[4] P. W. Higgs, Broken Symmetries and the Masses of Gauge Bosons, Physical Review Letters, 13, 508 (1964).

[5] F. J. Hassert et al., Physics Letters B, 46, 121 (1973).

[6] G. Arnison et al, Experimental Observation of Isolated Large Transverse Energy Electrons with Associated Missing Energy at $\sqrt{s}=540 G e V$, Physics Letters B, 122, 103 (1983).

[7] M. Banner et al, Physics Letters B, 122, 476 (1983).

[8] F. Abe et al, Measurement of W-Boson Production in 1.8 TeV pp Collisions, Physical Review Letters, 62(9), 1005 (1989).

[9] The CDF Collaboration and the DØ Collaboration, Physical Review D, 70, 092008 (2004). 
[10] The LEP Electroweak Working Group, 2003-01 (2003) http://lepewwg.web.cern.ch/LEPEWWG/lepww/mw/Winter03/mwgw_w03.ps.gz

[11] The CDF and D0 Collaborations, Combination of CDF and D0 Results on the TopQuark Mass, hep-ex/0404010, (2004).

[12] F. Halzen, A. Martin, Quarks and Leptons, John Wiley \& Sons (1984).

[13] G. Altarelli, Status of the Standard Model and Beyond, 38th Rencontres de Moriond, hep-ph/030605 (2003).

[14] The Super-Kamiokande Collaboration, Evidence for oscillation of atmospheric neutrinos, Nucl. Rev. Lett. 81, 1562-1567 (1998).

[15] A. Byon-Wagner et al, $W$ boson physics at hadron colliders, Review of Modern Physics, 73, 267 (2001).

[16] G. Kane, Modern Elementary Particle Physics, Addison-Wesley (1993).

[17] R. P. Feynman, QED, The Strange Theory of Light and Matter, Princeton (1985).

[18] D. Gross et al., Asymptotically Free Gauge Theories, Physics Review Letters 30, 1343 (1973).

[19] The LEP Electroweak Working Group, (as of June 2005) http://lepewwg.web.cern.ch/LEPEWWG/plots/zpole_2005/ Precision Electroweak Measurements on the $Z$ Resonance, hep-ex/0509008 (2005)

[20] H. Lai et al., Phys. Rev. D. 55, 1280, hep-ph/9606399 (1997). H. Lai et al., Eur. Phys. J. C 12, 375, hep-ph/9903282 (2000).

[21] A. D. Martin, R. G. Roberts, W. J. Stirling and R.S. Thorne, Parton distributions: a new global analysis, Eur.Phys.J. C 14, 133, hep-ph/9803445 (2000). 
[22] J. Collins et al., Back-to-back jets in QCD, Nuclear Physics B 193, 381 (1981).

[23] B. Abbott et al., Measurement of the W boson mass, Physical Review D, 58, 092003 (1998).

[24] Fermi National Accelerator Laboratory, Tevatron Department, http://www-bdnew.fnal.gov/tevatron/

[25] D. Acosta et al., First Measurements of W and $Z$ Cross Sections from Run II of the Tevatron Collider, Physical Review Letters. 94, 091803 (2005).

[26] D. Mohl, G. Petrucci, L. Thorndahl and S. Van Der Meer, Physics and Technique of Stochastic Cooling, Phys. Rept. 58, 73 (1980).

[27] The CDF II Detector Technical Design Report, FERMILAB-Pub-96/390E (1996); http://www-cdf.fnal.gov/

[28] R. Brun et al., GEANT: Simulation Program for Particle Physics Experiments. User Guide and Reference Manual, CERN-DD-78-2-REV.

[29] S. Agostinelli, GEANT4, A Simulation Toolkit, Nuclear Instrument Methods. A506, $250(2003)$.

[30] T. Sjostrand et al., PYTHIA 6.2 Physics and Manual, hep-ph/0108264, (2001).

[31] C. Balazs, et. al., Soft gluon effects on lepton pairs at hadron colliders, Physical Review D. 56, 5558 (1997).

[32] F. Landry et. al., Fermilab Tevatron run-1 Z boson data and the Collins-SoperSterman resummation formalism, Physical Review D. 67, 073016 (2003).

[33] F. Landry et. al., New fits for the non-perturbative parameters in the CSS resummation formalism, Physical Review D. 63, 013004 (2000). 
[34] B. Abbott et al., Measurement of the W Boson Mass, Physical Review D. 58, 092003 (1998).

[35] U. Baur et. al., Electroweak radiative corrections to $W$ boson production in hadronic collisions, Physical Review D. 59, 013002 (1998).

[36] I. Vollrath et. al., Event Generation for the Run 2 W Mass Measurement, CDF Note $7775,(2005)$.

[37] C. Calame et. al., Higher-order QED corrections to W-boson mass determination at hadron colliders, Physical Review D. 69, 037301 (2004).

[38] J. Pumplin et al., New generation of parton distributions with uncertainties from global QCD analysis, Journal of High Energy Physics 07, 012 (2002).

[39] A general-purpose framework for PDF analysis using PYTHIA and ROOT has been developed by Pasha Murat.

[40] A. D. Martin et al., MRST partons and uncertainties, hep-ph/0307262 (2003).

[41] K. Rinnert, A Fast-Access Material Map for the SVX II/ISL Silicon Tracking Volume at CDF 2, CDF Note 5846, (2002).

[42] Particle Data Group, Phys. Lett. B 592, 242 (2004).

[43] Particle Data Group, Phys. Lett. B 592, 813 (2004).

[44] Particle Data Group, Phys. Lett. B 592, 838 (2004).

[45] A. Kotwal et al., Identification of Cosmic Rays using Drift Chamber Hit Timing, Nuclear Instruments and Methods in Physics A506, 110-118 (2003).

[46] A. Kotwal, Muon Energy Loss and Momentum Scale Studies using Cosmic Rays and J/psi Events, CDF Note 6351, (2003). 
[47] Particle Data Group, Phys. Lett. B 592, 348 (2004).

[48] P. Sutton et al., Parton distributions for the pion extracted from Drell-Yan and prompt photon experiments, Physical Review D. 45, 2349 (1992).

[49] R. Rijken et al., Order $\alpha_{s}^{2}$ contributions to the Drell-Yan cross section at fixed target energies, Physical Review D. 51, 44 (1995).

[50] R. Hamberg et al., A complete calculation of the order $\alpha_{s}^{2}$ correction to the Drell-Yan K Factor, Nuclear Physics B359, 343 (1991).

[51] R. Harlander et al., Next-to-next-to-leading order Higgs production at hadron colliders, Physical Review Letters 88, 201801 (2002).

[52] W. van Neerven et al., The $O\left(\alpha_{s}^{2}\right)$ corrected Drell-Yan $K$ factor in the DIS and MS scheme, Nuclear Physics B382, 11 (1992).

[53] F. Abe et al., Measurement of the ratio $\sigma \cdot B(p \bar{p} \rightarrow W \rightarrow e \nu) / \sigma \cdot \operatorname{Br}(p \bar{p} \rightarrow Z \rightarrow e e)$ in p p collisions at $\sqrt{s}=1800 \mathrm{GeV}$, Physical Review D. 52, 2624 (1995).

[54] A. Kotwal, C. Hays, W. Trischuk, O. Stelzer-Chilton, I. Vollrath, L. Nodulman, Measurement of the W Boson Mass in Run 2, CDF Note 7104, (2005).

[55] D. Waters, private communication, (2005). 